NIST

PUBLICATIONS

\section{Navy Safety Center Data on the Effects of Fire Protection Systems on Electrical Equipment}

\section{Robert S. Levine}

U.S. DEPARTMENT OF COMMERCE National Institurte of Standards and Technology Building and Fire Research Laboratory Gaithersburg, MD 20899
Sponsored by:

Nuclear Regulatory Commisaion Washington, DC
U.S. DEPARTMENT OF COMMERCE Robert A. Mosbacher, Secretary MATIONAL INSTIUUTE OF STANDARDS AND TECHNOLOOY John W. Lyons, Director 



\section{Navy Safety Center Data on the Effects of Fire Protection Systems on Electrical Equipment}

\section{Robert S. Levine}

\author{
U.S. DEPARTMENT OF COMMERCE \\ National Institute of Standards \\ and Technology \\ Building and Fire Research Laboratory \\ Gaithersburg, MD 20899
}

April 1991

Sponsored by:

Nuclear Regulatory Commission Washington, DC

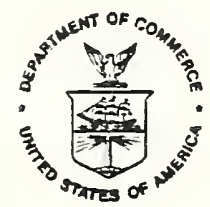

U.S. DEPARTMENT OF COMMERCE Robert A. Mosbacher, Secretary NATIONAL INSTITUTE OF STANDARDS AND TECHNOLOGY

John W. Lyons, Director 

NAVY SAFETY CENTER DATA ON THE EFFECTS OF FIRE PROTECTION SYSTEMS ON ELECTRICAL EQUIPMENT

NRC GENERIC ISSUE 57

\section{TABLE OF CONTENTS}

I ABSTRACT

$\underline{\text { PAGE }}$

II INTRODUCTION AND SIGNIFICANCE

III PRELIMINARY DISCUSSION WITH NAVY EXPERTS

IV RESULTS FROM THE NAVY SAFETY CENTER

V FINDINGS - - TECHNICAL RESULTS

(A) REASONS FOR FIRE AND FIREX MISHAPS 5

(B) INCIDENCE OF COLLATERAL DAMAGE BY WATER SYSTEMS 5

(C) INCIDENTS OF INADVERTANT OPERATION OF HALON AND CARBON DIOXIDE SYSTEMS

VI CONCLUSIONS

VII REFERENCES

TABLE 1, Reasons for fire extinguishing system mishaps

TABLE 2, Collateral damage - Summary

Appendix A, Relevant Naval Safety System Data

I. Shore Facilities

II. Submarines

III. Surface Ships

Appendix B, Inadvertant Operation of Halon Systems

Appendix C, Inadvertant Operation of CO2 Systems 


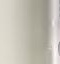


Navy Safety Center Data on the Effects of Fire Protection Systems on Electrical Equipment

Final Report to Nuclear Regulatory Commission - Generic Issue 57

By: Robert S. Levine

Building and Fire Research Laboratory

National Institute of Standards and Technology

I. Abstract:

Records of the Navy Safety Center, Norfolk, Va. were reviewed to find data relevant to inadvertant operation of installed Fire Protection Systems (FPS) in civilian Nuclear power plants. Navy data show that the incidence of "collateral" (additional accompanying) fire or other damage by fresh water on operating electrical equipment in submarines and in shore facilities is about the same as the civilian experience, about 30\%. Aboard surface ships, however, the collateral damage incidence is much lower, about 15\%. With sea water, the collateral damage incidence is at least $75 \%$. It is concluded that the fire extinguisher water has to be contaminated, for instance by rust in sprinkler systems or deposited salt spray, for most collateral damage to occur.

Reasons for inadvertant operation (or advertant operation) of FPS systems at shore facilities, submarines, and surface ships resemble those for nuclear power plants. Mechanical or electrical failures lead the list, followed by mishaps during maintainance. Detector and alarm system failures are significant problems at navy shore facilities, and significant at nuclear power. plants. Ships and submarines have few automatic FPS systems, so this kind of failure is not significant. Prompt action with a portable $\mathrm{CO}_{2}$ extinguisher is usually effective.

Fixed halon and $\mathrm{CO}_{2}$ systems in shore facilities cause no collateral damage.

Lists of individual Navy incidents with water and with halon and carbon dioxide are included as appendices to this report. 
II: Introduction and Significance.

Reference ( 1 ) lists instances of inadvertant operation of installed fire protection systems in US commercial nuclear power plants. About $30 \%$ of these incidents, if they involve water systems, cause collateral damage, even, when the water contacts operating electrical equipment, fire. This may damage safety circuits or equipment. Collateral damage is rare for $\mathrm{CO}_{2}$ systems, and non-existant for halon systems (2).

There is not a consistent set of reasons to account for the inadvertant FPS operation, although malfuctioning detector systems are sometimes involved.

Since the incidence of these mishaps is significant, the U.S. Nuclear Regulatory Commission is carrying out an evaluation of the effects of fire protection system actuation on safety related equipment. The purposes of the study related in this report are to enrich the list of reasons for inadvertant operation of fire protection systems, based on Navy experience, and to derive relevant information on the effects of fire suppressants on equipment.

Naval vessels resemble nuclear power plants in two respects. There is a large amount of installed electrical equipment, cabinets and switchboards, and there are numerous opportunities for these to make contact with water.

There are significant differences, too, between naval vessels and nuclear power plants. Generally fire extinguishment is carried out manually aboard ship by trained naval personnel. They first use $\mathrm{CO}_{2}$ extinguishers and then, if the $\mathrm{CO}_{2}$ is not adequate, water and/or dry chemicals applied through hoses. The water may come from a potable water main used for other purposes, but sometimes sea water is used. Sprinkler systems are rarely installed on Navy vessels. Similarly, automatic detector-alarm systems are not widely used, so we have little additional data from ships and submarines. The Navy historically depends on the presence of personnel in most spaces to observe any fire event, and to take early action. This situation is changing to a degree as new ship designs require smaller crews. Thus some spaces may not be manned in the future, and detectors and automatic systems may be more widely used on surface vessels and submarines.

\section{III: Preliminary Discussion with Navy Experts}

The method of search was first to query expert Navy sources on their experience, then search the records of the Navy Safety Center. The results obtained were discussed with relevant civilian experts. Each of these steps will be discussed in turn.

Two groups of Navy experts were queried. The first group was Dr. Homer Carhart and Dr. Frederick Williams of the Navy Technology Center for Safety and Survivability of the Naval Research Laboratory. They are responsible for research in fire and damage control. Some years ago a contractor to the Navy 
visited ships where mishaps had been reported to interview the personnel involved. The author compared the unpublished report that resulted with data from the Navy Safety Center. The narratives in the report do not seem more complete than the narratives in the Navy Safety Center records, lending confidence in the Navy reporting system.

The second group of Navy experts was the NAVSEA Fire Protection Division. This unit designs and specifies fire safety provisions for Navy ships. The author discussed nuclear power plant incidents with Mr. Robert Darwin, chief of that division. One Navy action that may be of interest is that they intend to backfit ships with hand-held 20 pound Halon 1211 fire extinguishers. These are very effective, cause no collateral damage, and because they seldom would be used, would have little impact on the Earth's ozone layer.

Despite its reluctance to install automated systems, the Navy does have automatic deluge systems in munitions magazines. These are designed to keep the munitions cool. They are actuated by a thermo-pneumatic (non-electronic) system, where an increase in air pressure caused by heating a tubular volume actuates a valve. Despite the intent to make these systems insensitive to the usual causes of false alarms, they have been actuated inadvertantly due to faulty maintenance.

The fresh water fire main on a ship is really a general purpose water main. Therefore, there are pressure surges in the main, and where installed sprinkler systems have been used, these surges sometimes caused valves to open. This has also happened in a nuclear power plant, where the water hammer as the line was pressurized caused water discharge ( 3 ).

Mr. Darwin stated that the choice of detectors is a key factor in avoiding unwanted discharge. There are detector designs that are very false alarm resistant. Typically these require two independent signals to confirm a fire. For instance, one design uses a response in the short wave length "solar blind" ultra-violet plus flicker in the infra-red. Both must occur. A welding arc, or lightning, could set off the first, but would not be likely to cause a sustained infra-red flicker. However, these detectors are expensive.

False alarms cause trouble. Naval personnel are likely to remove or deactivate a detector after the second false alarm. Generally, the Navy will not trust a detector to cause flow, but they may use it to dispatch a person to the site to investigate.

Mr. Darwin suggested we characterize whether the fault causing inadvertant operation is in the detector system, or in the mechanical system. Mr. Darwin also suggested querying the members of the NFPA 13 committee for their experience. NFPA (National Fire Protection Association) 13 is the standard for the installation, maintenance and use of sprinkler systems. He also referenced a presentation at a recent NFPA meeting, on inadvertant trips of fixed systems (see below). 
IV Results from the Navy Safety Center.

The Navy Safety Center, located at the Naval Air Station, Norfolk, VA, collects safety-related mishap data from the Navy, and since 1984 from the other uniformed services. The center has three separate sets of programs.

A - Shore Facilities. This includes warehouses, hangers, shops, etc. The contact is Mr. Vincent Lisa, 804-444-1187. The commander is Capt. R. D. Shoop, Jr.

B - Surface Ships. This includes pierside and yard maintenance, as well as ships underway. The contact is Mr. Alvin Becker, 804-444-1563. The commander is Capt. P. K. Glasier.

$C$ - Submarines. Again this includes pierside and other maintenance. The contact is Lt. Comdr. Greg Hempen, 804-444-5092. The commander is Capt. J.M. Rushing.

Each program has a separate Navy instruction on what to report and how to report it. For this reason the three sets of data are coded differently for retrieval.

The Shore Division instruction is OPNAVINST 5102.1C, dated 3 March 1989, as modified by a 22 May 1990 revision. This instruction includes the following:

"1) a DOD Fire Incident Report, DD Form 2324, will be submitted within 14 working days when:

(i) The ship is berthed at a shore facility and another Fire Department helps,

(h) - -operation of an automatic sprinkler system or other fixed extinguishing system as a result of a fire, or operation of an automatic sprinkler system or other fixed extinguishing systems at a DOD Installation for reasons other than fire, if damage results from such operation.

(o) Exclusions:

4. Operation of extinguishing systems on ships."

Other parts of the instruction caution that the type of material first ignited is often not the most sensitive material; requires that the avenue of flame travel be described, as well as the kind of agent and quantity used. In perusing a number of incidents, the author found that this designated data was usually useful to aid the Safety Center personnel to assign key words for retrieval. The instructions all call for a "Narrative", and these seem to be written by someone who is knowledgeable and is sensitive to the causual concerns important to this project. The one weak link is that the narrative writer sometimes does not make it clear whether fresh water or salt water was used. Generally this is apparent from the context of the event.

It was necessary to work at a computer terminal with the help of each of the 
key personnel above to derive a set of key words to yield the data desired. "Fire" was one key word, of course. "Narrative" statements were asked for and in some cases, additional data. In all cases an identification number that incorporates the date of the incident accompanies the computer printout. Although it is possible that some relevant cases were missed, the total number of cases was large. On the order of 100 cases were queried initially in each category, so the results are thought to be meaningful to the goals of the project. Late in the project, when additional experience on Halon and carbon dioxide systems was desired, shore facility data back to 1980 (about 500 more cases) were obtained.

\section{V) Findings--Technical Results}

Findings are presented here in three categories: "Reasons for Fire and Firex Mishaps", "Incidence of Collateral Damage by Water From Firex Systems" and "Incidence of Collateral Damage By Halon or Carbon Dioxide Systems". The appendices to this report list one year of inadvertant (no fire) incidents from shore facilities, 43 years of submarine incidents, 8 years of ship incidents, and 11 years of inadvertant halon and $\mathrm{CO}_{2}$ system operation in shore facilities.

\section{(A) Reasons for Fire and Fire Protective System Mishaps:}

Table 1 summarizes the Navy Safety Center Data, and compares it to NRC data from nuclear power plants.

It is seen that actuation by human error and by false alarms are minor considerations in the problem of advertant or inadvertant operation of fire protective systems, 88 to 148 , except for submarines. This is true for both Navy experience and nuclear power plant experience, and is different than the experience and opinions of civilian fire protection engineers (4). Of course, in the surface ship and submarine data there are few fixed extinguishing systems, so there is little chance for this problem to occur.

Leaky pipes, for active sprinkler systems, most because of patches or attachment of branch lines, are a small but significant problem (about $10 \%$ ). Considering that the hardware technology for installed systems is mature, this is surprising.

The big problems are malfunctioning of equipment (25-50\%) and mistakes during maintenance (20-508 of the cases). Obviously, if equipment rarely malfunctioned, there would be little need for FPS. A system with no other problems would show that equipment malfunctions would be $100 \%$ of the reasons for mishaps. So ship systems ( $49 \%$ malfunctions) are as good as those in nuclear power plants ( $48 \%$ malfunctions). By this reasoning, submarines (35\% equipmene malfunctions) and shore facilities (26\%) have a larger share of "other problems". The other problems are primarily maintenance. Only 198 of the nuclear power plant mishaps are caused during maintenance, but $51 \%$ of the submarine mishaps and 348 of the ship problems are maintenance. 
In one way the submarine situation can be classed as a testimony to excellent equipment reliability. It took 43 years to accumulate 95 fire-related incidents. The 42 nuclear power plant incidents occurred during 10 years, the 112 ship incidents in 8 years, and 68 shore facility incidents in one year.

The author's conclusions from these data are that the Naval vessels do resemble nuclear power plants, but that maintenance mishaps are less likely in nuclear power plants.

(B) Incidence of Collateral Damage by Water from Fire Protection Systems.

A summary of damage when water comes into contact with operational equipment, is shown in Table 2. First of all, it should be noted that damage is highly likely when sea water is involved ( $75 \%$ damage). However, even though there are ways that fresh water on Navy ships can pick up some salt from the surfaces of equipment, fresh water on Navy ships is less than half as likely to cause trouble as fresh water in nuclear power plants (15\% vs 30\%). The incidence on submarines shows as $28 \%$, but there are only 5 cases, and the writer's criteria were very conservative. For instance, included is a case where steam cleaning a generator caused a fire. This was ascribed to fresh water even though there was obviously dirt on the generator that was blown into it.

The Philadelphia Naval Shipyard has a particularly successful record of refurbishing electrical equipment that had been wet with water. The superintendant of the electrical shop (5) said that he could not remember a case where a shorting-electrical problem had been caused by fresh water. The only problem experienced at the shipyard with fresh water was corrosion if drying the equipment was delayed. This was blamed on salt that had been deposited on the equipment. The superintendant suggested that the water in the nuclear power plant fire protection systems was contaminated.

It is well known that sprinkler systems are likely to have substantial amounts of rust and other contaminants in them. This can contact energized electrical equipment when the system is discharged. Sprinkler systems are tested with water when installed, then closed up. The NFPA standard calls for flushing once a year, but even in a year rust can occur.

When fresh water is used for fire fighting aboard ships, it comes from a potable water main. The water quality meets Bureau of Medicine criteria. It is distilled aboard ship, then stored in a lined steel tank and distributed in copper or cupro-nickel pipe (6). From 0.2 to $2.0 \mathrm{ppm}$ of elemental bromine are added, depending on the purity of the original sea water (7). The final water contains less than $10 \mathrm{ppm}$ of dissolved solids. When this water is used on a fire, it is discharged through rubber lined hose which, of course, adds no rust. 
(C) Incidents of Inadvertant Operation of Halon and Carbon Dioxide Systems.

As mentioned above, 11 years of Navy Safety Center data on inadvertant discharges of halon and $\mathrm{CO} 2$ have been obtained and examined. This additional data are valuable because there are only 17 such events in nuclear power plants, and additional data aid in assigning a not overly conservative probability of collateral damage. The table below summarizes the data:

\begin{tabular}{|c|c|c|c|c|}
\hline$\frac{\text { Year }}{1990}$ & $\frac{\text { Halon cases }}{14}$ & $\frac{\text { Damage? }}{\text { no }}$ & $\mathrm{CO}_{2} \frac{\text { cases }}{4}$ & $\frac{\text { Damage? }}{\text { no }}$ \\
\hline 1989 & 24 & no & 1 & no \\
\hline 1988 & 18 & no & 2 & no \\
\hline 1987 & 18 & no & 3 & no \\
\hline 1986 & 13 & no & 5 & no \\
\hline 1985 & 23 & no & 1 & no \\
\hline 1984 & 6 & no & 3 & no \\
\hline 1983 & 1 & no & 4 & no \\
\hline 1982 & 0 & - & 2 & no \\
\hline 1981 & 0 & - & 2 & no \\
\hline 1980 & 1 & no & 5 & no \\
\hline Total. & $\overline{118}$ & & 32 & \\
\hline
\end{tabular}

It can be seen that no collateral damage occurred as a result of Halon or $\mathrm{CO}_{2}$ discharges. The reason for few data on halon systems prior to 1985 is that the Navy decision to use halon was made about 1980, and some time was then required to design and install systems.

\section{(VI) Conclusions}

The reasons for inadvertant operation of installed fire protection systems contained in the Navy Safety Center Data resemble those for nuclear power plants. Therefore there is support for utilizing this experience in an analysis of the probability that damage from inadvertant operation can occur in nuclear power plants (NRC Generic Issue 57).

Examination of the incidence of collateral damage, including fire, that can occur when water used in protection systems makes contacts with operating electrical equipment shows that Navy shore facilities are similar to nuclear power plants $(30 \%)$, but that ships have only about half the incidence of damage when fresh water is used (15\%). Sea water, on the other hand, gives at least a $75 \%$ incidence of damage. Fresh water on a ship is very pure, whereas water sprinkler systems are very likely to have large amounts of rust and other impurities in the first water to be discharged. So it is concluded that a large part of the collateral damage when installed water fire protection systems are inadvertantly discharged is due to impurities in (and high conductivity of) the water. 
Confirming the relatively few cases in nuclear power plants, discharge of halon or $\mathrm{CO}_{2}$ systems in shore facilities causes no collateral damage of the kind of concern here. Because $\mathrm{CO}_{2}$ systems can be massive, there are cases where personnel are temporarily overcome due to lack of oxygen. It is possible that an unconscious person might not be discovered in time to be rescued. The Navy data do not indicate any incidents where the chilling effect of the cold $\mathrm{CO}_{2}$ is a factor.

\section{References:}

(1) Lambright, John A., Brosseau, D., Bohn, M.P., Simion, G.P., Sattison, M., " Evaluation of Generic Issue 57, Effects of Fire Protection System Actuation on Safety-Related Equipment" NUREG/CR-5580, SAND90-1507, EGG-NTA-9081 (June 1990)

(2) Lambright, John, Briefing Charts, GI-57, "Effects of Fire Protection System Actuation on Safety-Related Equipment" Sandia National Laboratories, June 22, 1990

(3) NRC Information Notice 87-14, (Event occurred at Cooper Nuclear Power Station, April 4, 1984)

(4) Personal Communication, Michael L. Brookins, Manager, Property Loss Prevention, Norfolk Southern Corp., Roanoke VA., to Robert S Levine, Memo to File Oct 11, 1990

(5) Personal Communication, Mr. Gilbert Ward, Superintendant, Electrical Shop, Philadelphia Naval Shipyard, (215-897-3655) To Robert S. Levine, Memo to file, Dec. 14, 1990

(6) Naval Ships Technical Manual S9086-SE-STM-010/ Chapter 533, "Potable Water Systems" (Jan. 1986)

(7) Personal Communication - Mr. Greg Johnson, Everpure Corp., Westmont Illinois, to Robert S. Levine, Memo to File "Navy Potable Water on Surface Ships" April 24, 1991 


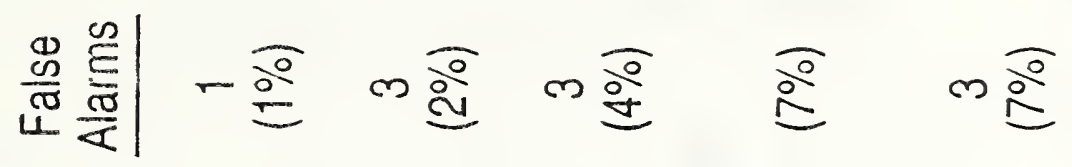

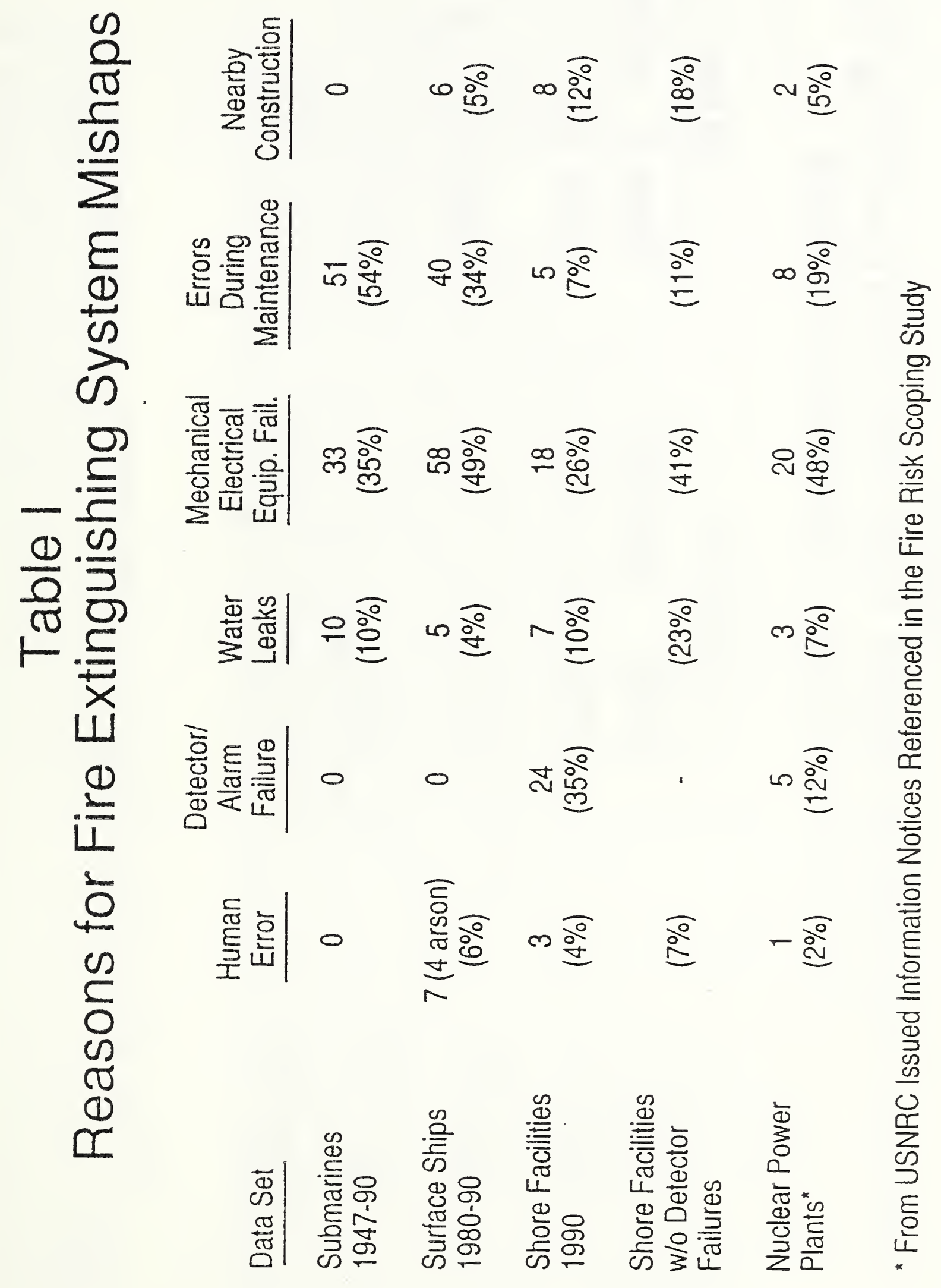




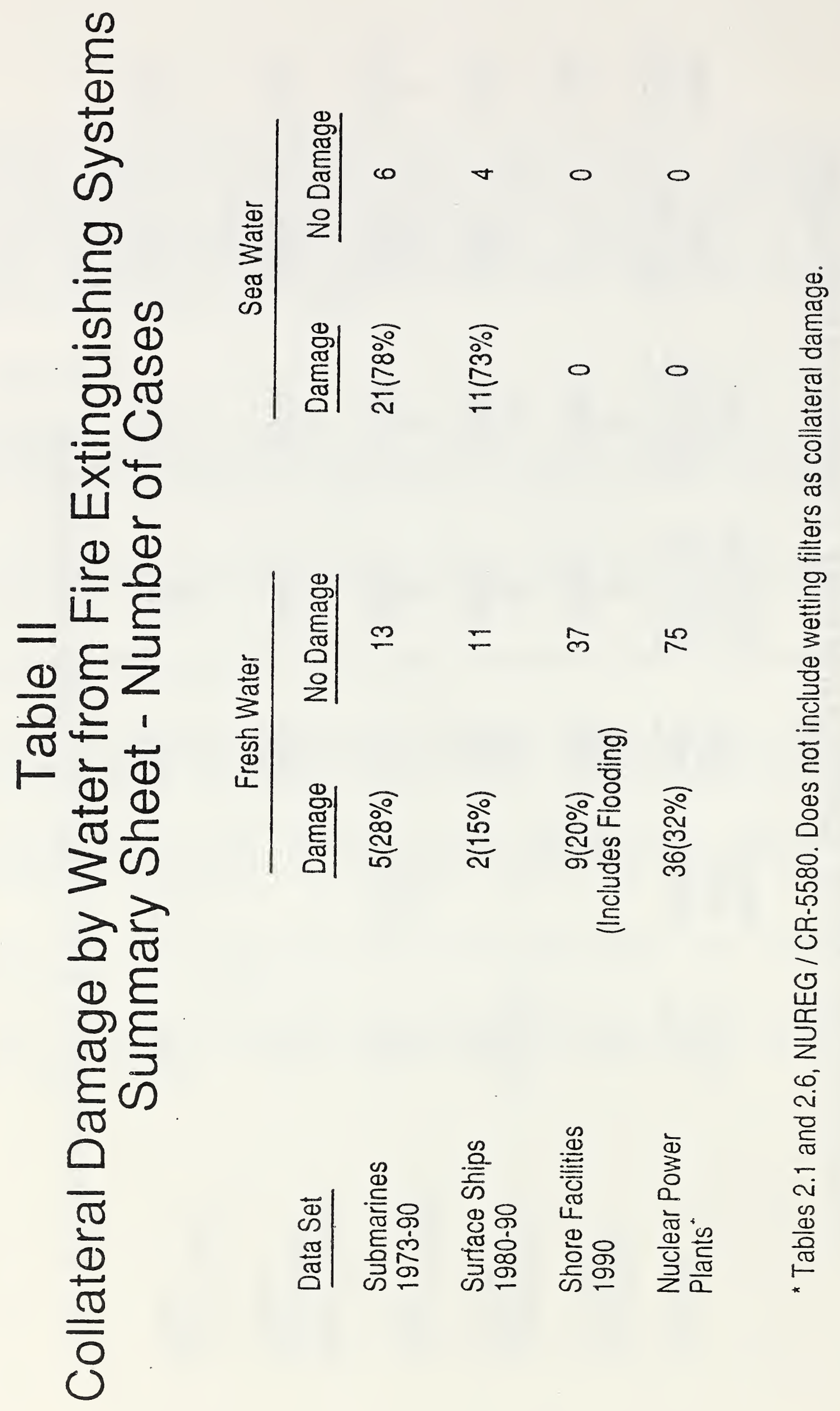


I. Shore Facilities

Record I.D.

90010290600

90011691600

90012392000

90012590200

90020290800

90020791100

90021190100

90021290400

90021590200

90021591000

90021890200

90021891200

90022491100

90022690100

90022790600

90031590300

90031290900

90031490900

90031991000

90032191600

90032490200

90032590600

90033090400

90040991800

90041290500

90041790900

90041791500

90042190300

90042990700

90050190700

90050490200

90050491200

90050591200

90050790100

90050791500

90050991600

90051690300

90051691000

90051991000

90052590900

90060190900

90061090100

90061291100 $\underline{\text { Reason }}$

Slow leak in Deluge System

Condensation in Pneumatic System

Defective Control Valve in Deluge System

Defective Sprinkler Head

Broken Sprinkler Pipe

Patch Pipe Sleeve Failed

Operated Pull Box by Mistake

Sprinkler Head Link Failed

UV Detector Saw Lightning

Relief Valve Froze - Housing Cracked

False Alarm from Heat Detector

Used Cigarette Lighter to Locate Switch

Opened Doors - Temp. Change Detected

Sprinkler Head Broken by Lift Truck

Frozen Pipe Above Sprinkler Head

Bad Packing - OS\&Y Valve

Hoister Hit \& Fractured Sprinkler Pipe

Construction Crew Broke Sprinkler Pipe

Ran Fork Lift into Sprinkler Pipe

Faulty Rate of Rise Detector

System Activated by Persons Pulling Cable

Switch Operated when Power Off - Then Power Came On

Defective Link in Sprinkler Head

Source of Hot Gas Actuated Sprinkler Head

Leaky Pipe - Flooded Work Area

Automatic Alarm - Deluge System - False Alarm

False Alarm When Power Restored

False Alarm - Repair Technician Error

False Alarm - No Cause Found

Alarm System Pressure Switch Sensed Hose Motion

Leak Through Sprinkler Branch Line Connection

Maintenance on System Activated It

Overheating of 2 Heads by Incinerator

A/C Element Overheated - Produced Smoke

Switch Pushed by Mistake During Maintenance

No Cause Found, Deluge Abort System Not Working

Smoke Detector Actuated by Maintenance Working on $\mathrm{A} / \mathrm{C}$

One Sprinkler Head Actuated - No Reason Found

Accidental Operation of Remote Pull Cable - Contraction

Hot Air Leak Impinged on Sprinkler Head

Old (1944) Sprinkler Head Failed, FD Notification Failed

Short in Electrical Connector Activated System

Dust from Construction Activated Smoke Detector 
90062191100

90062890500

90062890600

90070291500

90071090700

90071091200

90071291200

90071291100

90071791700

90071890400

90071990300

90072090300

90080291200

90080390400

90081190500

90081690100

90082890800

90083090500

90090190200

90090590600

90091490500

90091790200

90092590100

90092690400

90092890700

90102590500
Bad Switch on Computer Power Supply - Smoke

Slow Leak in Deluge System

Stove System Powder Accidently Actuated

Faulty Heat Detector Actuated by Engine Vibration

Pull Box Mistakenly Actuated - Instructions in Error

Loose Grounding Wire Shorted Others in Electrical Box

Fusible Link Melted Over Flame Grill

UV System Actuated During Elect. Storms \& Power Failure

Chip Actuated When Power Restored

Forklift Hit Piping - Pump Room Locked

Smoke from Exercise Carried to Detectors by Ducts

Computer Power Supply Overheated

AVC Pressure Switch Failed, Vapors Act. Smoke Detectors

Hood Fusible Links Replaced by Lower Temp. Links

Sprinkler Head $\left(165^{\circ} \mathrm{F}\right)$ Actuated by Heat Below Roof

Sprinkler Pipe Broken by Forklift

Heat from APU Activated Heat Detector

16 Inch Line Broke Beneath Floor when Pressurized

Kitchen System Activated by Strain on Trip Wire

UV Detectors Activated by Arc Welding - Construction

Sprinkler Head Activated by Open Incinerator Door

UV Detectors Sensed Nearby Lightning Strike

4 Inch Sprinkler Line Coupling Failed

Sprinkler Head Link Failed

Dust and Humidity from AVC Startup Actuated Detector

Power Surge Caused Fire in Motherboard Panel 
II. Submarines

Record ID

730711D1701

$900911 \mathrm{~B} 0101$

891119B0601

890925B0901

$890728 B 1301$

890329B3001

$881117 \mathrm{~B} 2201$

880828B0901

880424B0401

871006B1501

$870522 \mathrm{~B} 3101$

$870218 B 0401$

870109B1401

860807B0701

$860729 B 0601$

860626B5601

$860520 B 5601$

851220B0201

851104B0901

850511B2101

850505B2201

850312B2801

841103B0301

841018B5301

840629B0301

840611B6801

840523B4801

840501B2001

840330B2601

$840321 B 5601$

840312B0501

$840217 \mathrm{~B} 3101$

840208B4201

840124B0401

\section{Reason}

Acetone Used to Clean Tank - Ignited by Broken Light

Hot Work in Navy Yard - Ignited Debris

Hot Work in Navy Yard - Ignited Protective Cover

Hot Work in Navy Yard - Ignited Wire Bundle

Hot Work in Navy Yard - Ignited Litter

Antifreeze Leaked Thru Valve into Breakers

Water (Salt?) Leaked onto Pump Motor

Oil Soaked Logging on Diesel Exhaust Ignited

Corroded Stuffing Let Sea Water Leak on Busbars

Hot Work in Navy Yard - Ignited Hull Insulation

Amine Leaking into No. 2 CO2 Scrubber Heater Section and Ignited Fire

Uncontained Sparks from Grinding Ignited Hull Insulation Combustibles

Sparks from Welding Dropped to Engine Room and Ignited Lagging

Hot Slag from Carbon-Arcing Escaped and Ignited Dust and Lint Combustibles

Hot Slag from Burning Operations Ignited Ship Hull Insulation Combustibles

Welding Sparks Ignited Debris

Steam Wetted Condensate Pump Motor Caught Electrical Fire When it was Started

Condenser Sea Water Hose Broke Loose Spraying Pump Motor and Caused Electrical Fire

Water in the Fan Plenum Caused Electrical Fire

Welding Sparks Landed on Sound Silening Material Which Started Smoldering

Sea Water from Bucket Accidently Poured Over Switchboard and Caused Electrical Fire

Hot Slag Ignited Foam Insulation in the Engine Room

Welding Slag Ignited Paper Towels, Wood Insulation, and Cable Insulation of Sail Structural

Welding Slag Ignited Electrical Wire, Rubber Insulation, Oil Grease Inside Missile Tube

Hot Slag from Welding Operation Ignited Hull Insulation of Torpedo Room Welder's Torch Ignited Hydraulic Oil Spraying from a Ruptured Hydraulic Oil Line

Welding Slag Ignited Rubber Insulation of Aux Tank

Welding Slag Ignited Bilge Rags in Engine Room

Welding Slag Ignited Bilge Rags in Torpedo Room

Malfunction Failure of Heater Power Indicator and Temperature Limiting Device Caused Electrical Fire

Slag from Grinding Ignited Oil Exposed Fiberglass Insulation Under Maneuvering Room Deck

Grinding Sparks Landed on Pile of Paper Towels and Caused Fire

Cutting Slag Ignited Flammable Material on Main Ballast Tank

Welding Slag Ignited Containment of Battery Well 
831123B2501

831122B2401

830901B4701

$830818 \mathrm{~B} 5601$

830808B6601

830725B7001

830630B4701

830416B2701

830202B0101

$811216 \mathrm{~B} 3201$

811203B0401

811021B1601

810922B4501

810828B1001

$810712 B 1201$

$801219 B 2401$

800322B1101

790417B1101

790104B1001

770712B3601

770118B2501

730221D0401 720927D0701

710621D0401

710609D0601

710106D1001

700829D0901

700625D0201

700416D0401

8l1128B1401

810520B4501
Air Arc Welding Slag Ignited Paper Trash - Flame Spread to Insolite Hull Insulation and Electrical Cable

Hot Slag in Radio Room, Ignited Lint and Dust Accumulated in Ventilation Pipe

Welding Slag Ignited Vent Filters in Laundry Room

Heat from Hot Welding Slag Ignited Oil Soaked Lagging from Engine Room Voltage Tester Probes Inadvertently Shorted Energy Terminal Lugs and Caused Switchboard Fire

Welder Sparks Ignited Spilled Flammable Liquid in Bow Area

Brine Dilution Tank Overflow, Stator Windings Shorted/Grounded and Caused Fire

Welding/Grinding Slag Metal or Sparks Ignited Combustibles in Sonar Dome Hot Slag from Silver Brazing Ignited Cork Insulation in Operations Compartment

Sparks from Welding Rod Ignited Insulation in Sail Bridge Trunk

Carbon Arc Welding Slag Ignited Insulation in Fan Room

Flame from Welder's Oxygen-Acetylene Cutting Torch Ignited Bale of KimWipe Paper Towels

Sparks from Grinding Metal Ignited Fibrous Glass in the Fan Room

Water Dripped into Transformers Shorted and Caused Fire in the Fan Room Battery Well Ventilation Filter Fell on Heater Element and Caused Fire Sparks from Grinding Ignited Insulation in Fan Room

Switchboard Wiring Shorted, Caused Excessive Current Draw and Electrical Fire

Fuel Oil Sprayed into Motor Generator Set and Caught Fire in Engine Room Fire Resulted from Arcing Due to Improper Tightened Busbar in Engine Room

Switchboard Fire Due to Sea Water Entering from Escape Trunk Hatch Through Fasteners in Top of Panel

Super Structure Fire Due to Hot Slag from Welding Zincs Ignited Nylon Mooring Line

Welder's Sparks or Slag Fell Behind Switchboard and Ignited Oily Dust

Fuel Oil Pressure Gauge Line Parted, Spraying Oil Contact with the Hot Engine Exhaust Header and Started Fire in Engine Room

High Resistance Connector Between Busbars and Breaker was Loose and Caused Electrical Fire in Machinery Room

Contacts were Cocked on the Cam, Caused Arcing and Fire in the Machinery Room

Electrical Cable Vibrated Loose, Shorting, Arcing and Caused Fire in Engine Room

Improper Storage of Combustible Materials in Gęnerator Room, Insufficient Facts to Establish Cause

Welding Hot Slag Fell into the Ventilation Duct and Ignited Dust and Dirt Near Intake

Welding Slag Ignited Plywood Disc Which Fell Down Conning Tower and Caused Fire

Motor Grounded from Water Spray Causing Arcing and Fire

Sea Water Spray from Failed Mechanical Seal Caused Pump Motor Short Out and Fire 
781127B1201

781013B2301

$780228 B 3801$

770920 B3701

760627B1701

730529D0501

720531D1001

710607D0901

700413D0701

900126B0401

860730B0401

840517B5001

840408B1601

830410 B3501

821011B0101

820828B1201

811019B4901

800516B3401

800318B5001

800208B0701

800103B4701

790521B4701

790326B0901

790211B1001

790122B1701

780726B4201

780408B1701

780301B4001

740810D0701

730327D0401
High Pressure, High Temperature Brine Discharged into Pump Motor Causing Ground and Fire in Engine Room

Water Vented from Cooler Splashed into Motor Causing Ground and Fire in Engine Room

Leaking PKP Caused Commutator and Brush Arcing

Sea Water Leaking into Motor Casing and Windings in Engine Room

3000 PSI Plug Blew Out and Damaged Nearby Piping

Condensation Water Shorted Rings and Rotor Bars of the High Pressure Brine Pump

Loose Spacer in Vent Piping Fell into High Pressure Brine Pump Motor

Check Valve Installed in System Backwards and Caused Overpressurization of Piping

Improper Valves Were Installed and Resulted in Blow Off the Union Nut

200 Gallons of Water Spilled into AMR

200 Gallons of Oily Water Came into Galley and Torpedo Room Due to Failure in Flushing Line

Sea Water Entered Battery Well and Resulted in Battery Discharge, Explosive and Toxic Gases

Engine Room was Flooded from Evaporator Strainer Failure

Engine Room was Flooded Due to Gland Seal Supply Line Failure

Engine Room Flooded Due to Improper Tag Out of Condenser and Absorber of Air Conditioning System

Faulty Hot Water Heater Relief Valve Stocking Open and Resulted in Flooding in Storeroom

SD-7 Body Blew Out, Spraying Sea Water and Flooded Both the AC and DC Ends

Lower Level Engine Room Flooded with Sea Water Due to Undrained Waterway and Improper Fitted Plugs

Normally Shut Libr Condenser Drain Valve was Open, Sea Water Sprayed onto Motor Generator, Shorted the Commutator and Brush Rigging

Flooding AMR Lower Level Due to Flood and Drain Valve Lifted from its Seat in Ships Trim System

Diesel Sea Water Hull Backup Valve Could not be Shut, Flooding the Machinery Room, Shorting the Switchboard and Resulted in Switchboard Fire

Drain Funnel For Condenser Overflowed onto ASW Pump Room and Flooded Pump Motor

50 Gallons Water Flooded into AMR Due to Depth Control Tank Manhole Cover Malfunction

Torpedo Tube Manhole Cover Opened, 100 Gallons of Sea Water Entered Torpedo Room

Drain Tunnel Above Pump Overflowed, Sea Water Enter and Short Out Motor Windings

O-Rings Failed and Causing Fresh Water Leak into Angle Solver Section

Plug in the Hydraulic System Blew Out, $20 \mathrm{Gal}$ of Oil Gushed into ERU and onto DOA Components in Engine Room

A Small Leak from a Pipe Union, Caused Water Run onto the Motor, Motor Windings were Damaged

Sea Water Flooding, Motor Generator Grounded

Diesel Generator Flywheel Coupling Guard Plug Missing. Water was Leaking Out the Generator Air Cooler Casing 
The Diesel Sea Water Overboard Backup Valve was Opened by Accident. Sea Water Splash Resulted in Grounding of Vital Electrical Panels

720403D0501 Overfilled Fresh Water Tank and Flooded the Void Space

710311D0801 A Pin Hole in the Compensating Water Piping in Maneuvering Rod Caused Flooding in Maneuvering

690903D0401

Flooding in Lower Level of Machinery Room was Due to Removal of a Hull Blanking Flange to Prevent Sea Water Leakage 


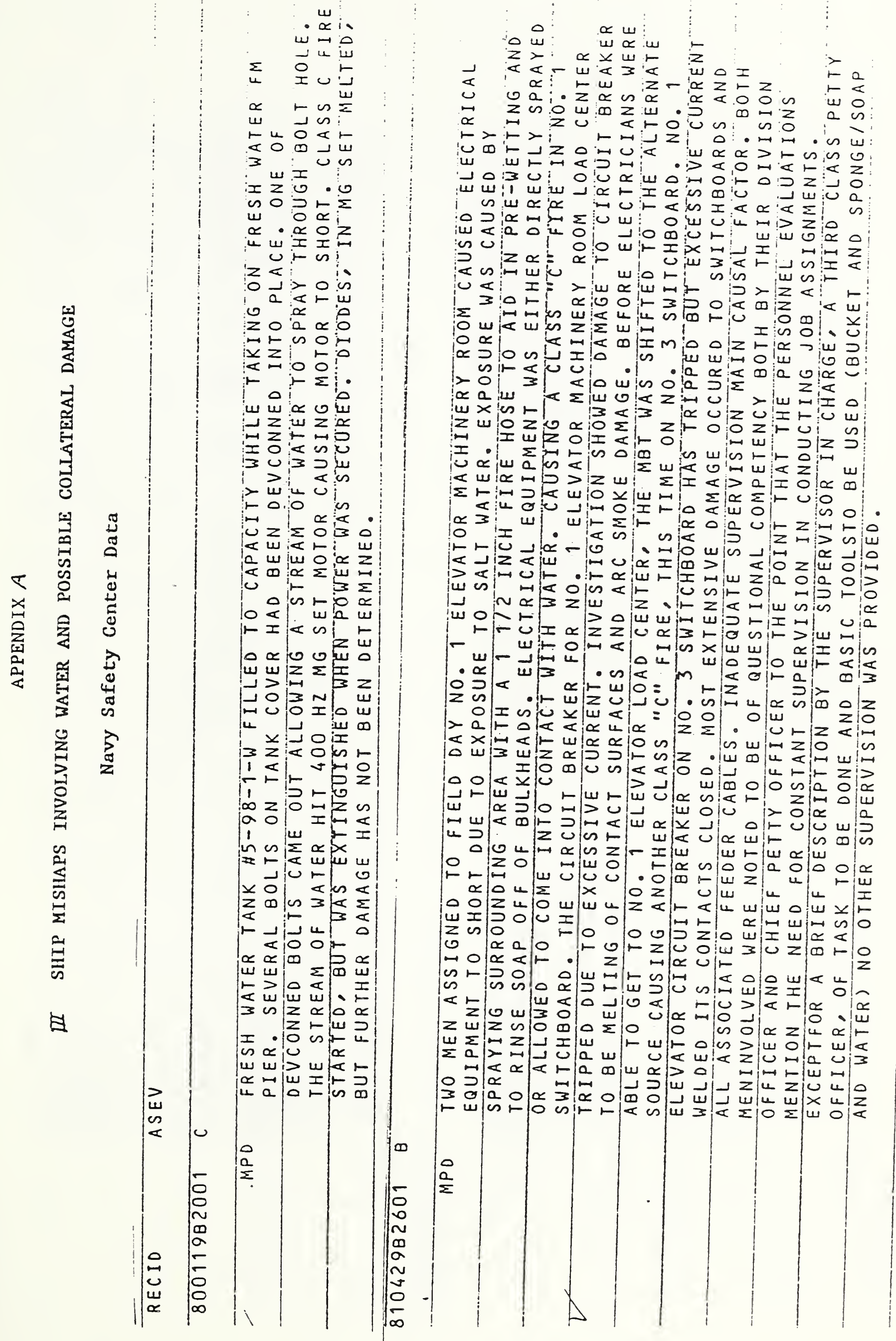




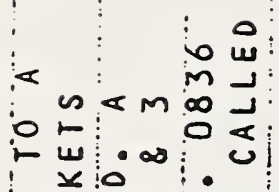

to u< 乎的比向 - c. I 0 o

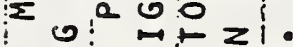

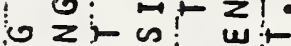
in w 1 u v in w

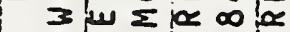

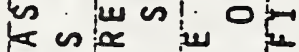
ए $\frac{\alpha}{3} \infty$ 些. $3 \ll \mu \infty$ a in $\infty$ il $<0$ $0 \geq 10$ 它 $\sum$ ! $\propto \frac{0}{0}<0$ ba $=0$. zolwa w o war an $F \leftarrow a$ in $\frac{a}{\alpha}$

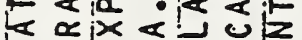

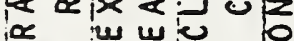

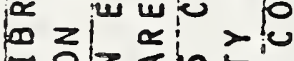
- 0 < < in $1:$

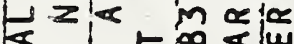
u in 40 a

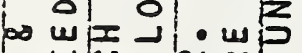
wes no w 420 t 20 (口) $<$ ru o o sle o $\supset \propto$ uा $2 \supset$ onמ - zur mas do o 0 o 织品

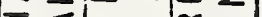
原 I s

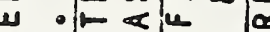

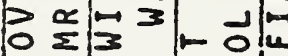
< us

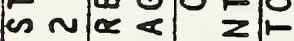
0 ख $20-0$ a w $\begin{aligned} & b \\ & 1\end{aligned}$ $0>\omega<$ u D 0 u w a $\infty$ a I 《传 n $z$ o \& $>\curvearrowleft 0 \propto \sum \vdash$

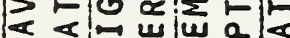

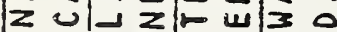
O $=$ a $z-1<<<\ldots$

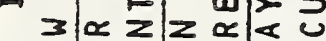

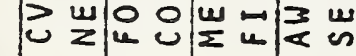

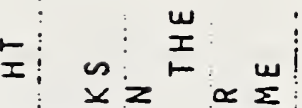

$w z \alpha=\mid \omega<$

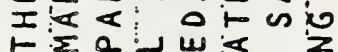

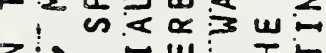
때 U ए w I 0 $0>0<\omega .3 m$ $\propto \propto \Sigma \curvearrowleft$ 은후

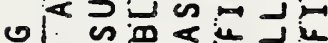

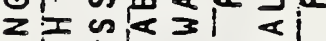

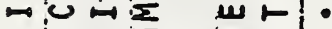
江江的山

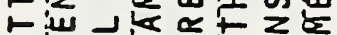
万ा

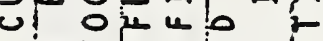
ำเ

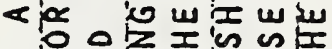
$x=2 n \leftarrow$ $F<5<0$

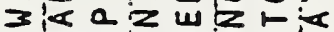
- 0 이니 n w《r: U⿴囗十丁 $\propto z \geq u \mid \alpha \propto x$ ol w 禹 $\begin{aligned} & \alpha \\ & 3\end{aligned}$ 구의 앙

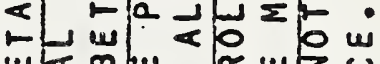
w

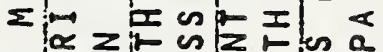

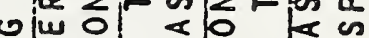

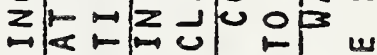
$\propto \Sigma m$. $\supset$ L Dus ox U1 $\ll, a \quad z<\sum \infty 0$

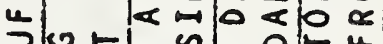

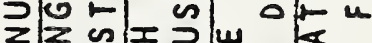
<纱之心

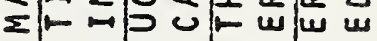
$5<0 \quad 5 z$ s) 3 ए u 10 a $z 0<0$ o 200

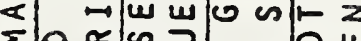
$\Sigma 00 \quad \propto ⿻ 上 丨$ < wins as $0 \geq \sum$

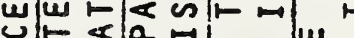

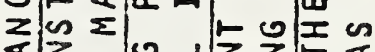

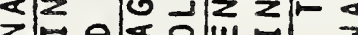
w w 0 जю. $\vdash$ - a c 1 d $z a$ a a Ju w

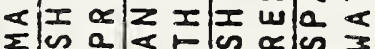
ر ल z

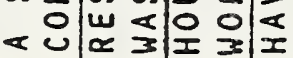

a

a

a 
\begin{tabular}{l|l|l|l}
$\infty$ & $\infty$ & $n$ & $\vdots$ \\
$\infty$ & $\infty$ & $n$ & 0
\end{tabular}

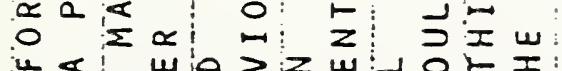

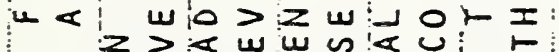

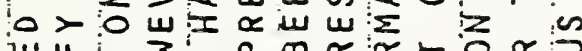
iw U口

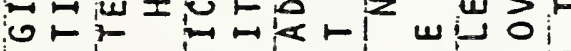

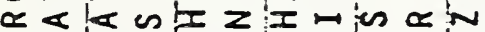

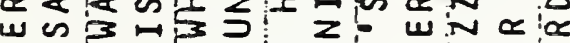
i 3 r w wol口

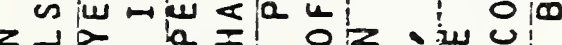

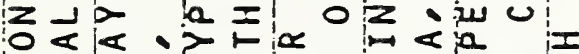

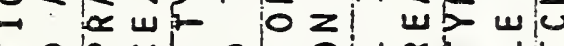
- ola ul o 0 a $<$ a w w w w w w w

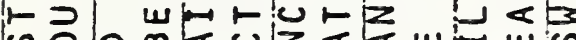

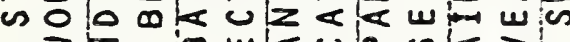

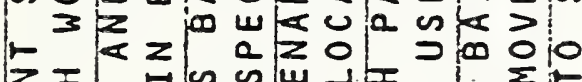

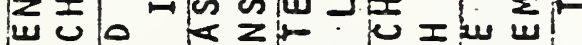

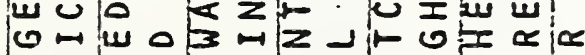

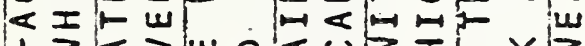
$13 k>$ w $0 \leqslant 0\}$

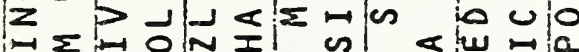

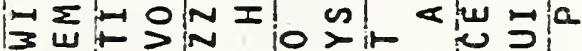
I ロ

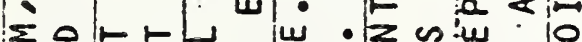

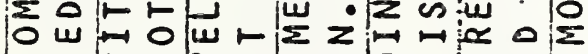
$0 u z z><\omega 0$ u w

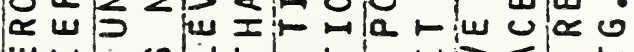

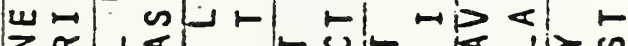

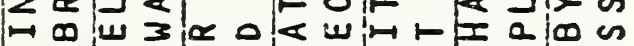
四 z w w a

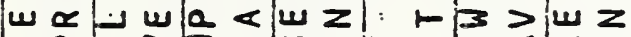
a $z$ 乎 $>0$ I n.I.

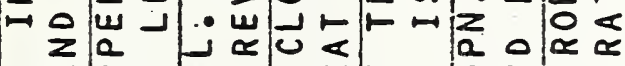

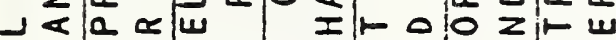

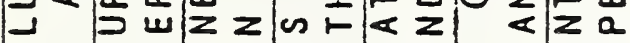
I0 a 0 o 0 I 0 o 0 o ฯ山س

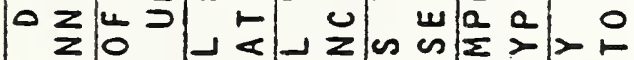

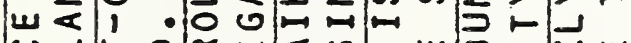

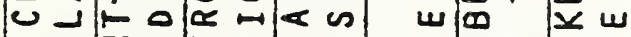

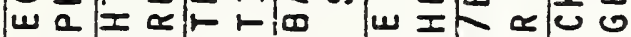

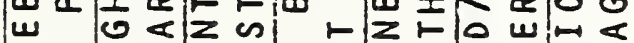

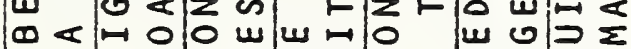

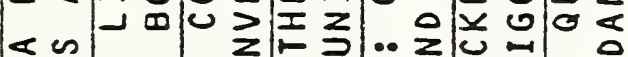

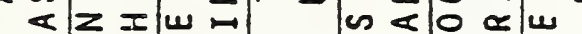
$0300 \Sigma$ o $z$ a

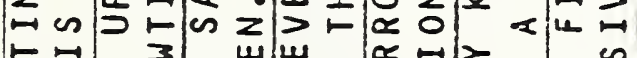
UI. us a 군

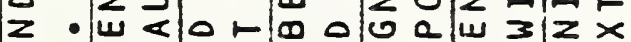
Z

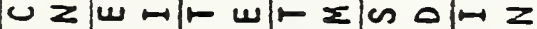

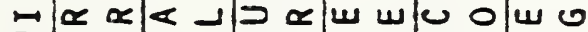

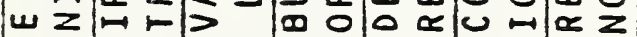
$山 \curvearrowleft \supset$ U

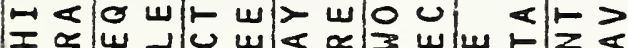

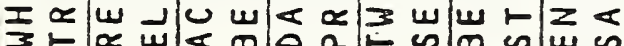

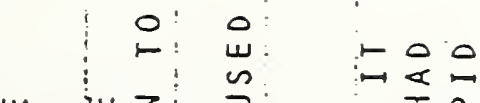

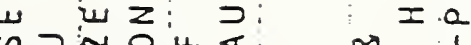
心 I I v ト

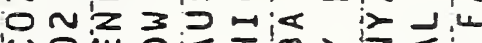
U i b J : w w 0 t $2 z z<! \Sigma=$

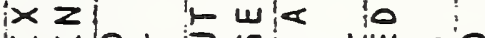

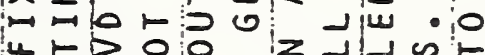
" - w w

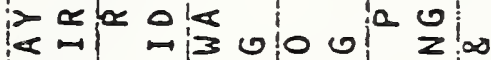
$\left\{\begin{array}{lllllll}3 & 0 & 0 & 0 & 0 & 0 & 2\end{array}\right.$

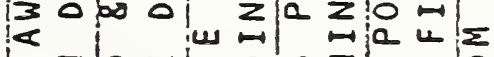
i zlo wla 0 lo zi

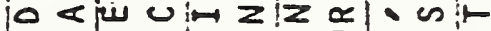
u It口iu $\omega \vdash k>\quad 3 a r w=0$

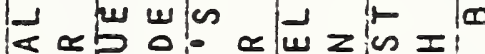

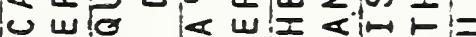
I

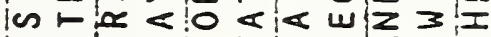

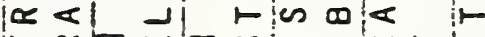
$r_{0} \propto z_{0}$ w N zon w

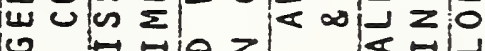
v I

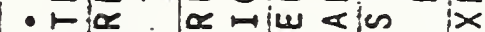

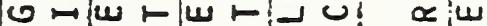
o 3 a כ is $\infty \mid z \omega<\rfloor$ s $\omega$ o U oll z $3<0$ will olo wia vin $\not$ a $N$ N $\propto$ U U

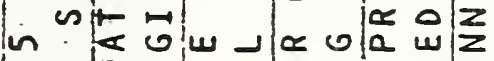
$\infty$ ol $\infty$ \& - سن $0<z 10$ a

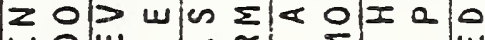

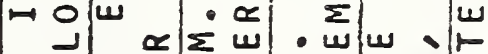
o - w 난 a $-<<001001$ w $\supset a \supset|z w| \vdash>$ I 0

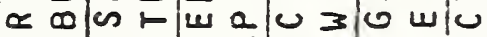

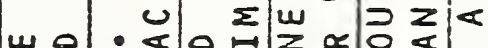
$\propto w \propto \quad z \propto \geq w$ wII $\mapsto N 0$ zu Ulo

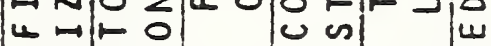

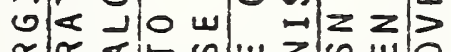
$\omega \propto \propto ⿻ 上 丨 \cos z$ en $w 0$ 川

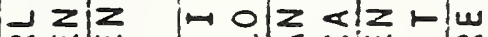
व

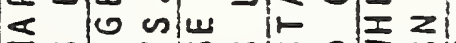

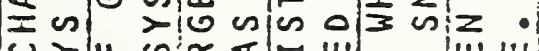
n n <w $w$ Jin $\alpha$ n w o!

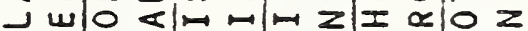




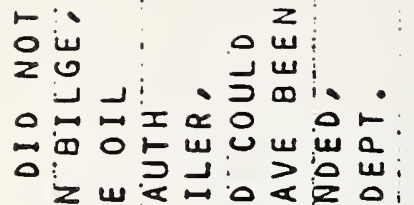

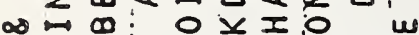

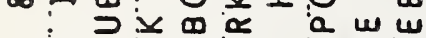

D 0 a

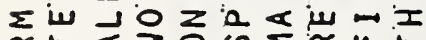

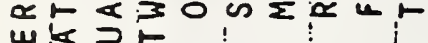

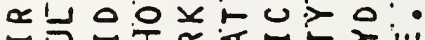

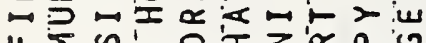
$z$ u w w - U ๙

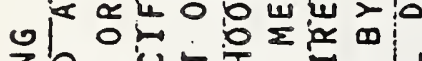

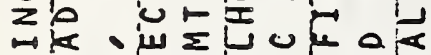
or wn<w

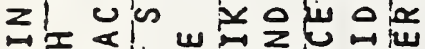

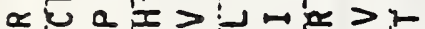
of us a $\Rightarrow$ क 0 la 红 5 w w w w $\propto$

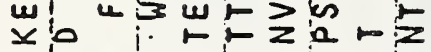

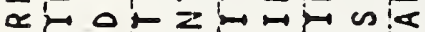
울 is ov un of w in

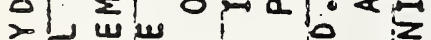
a 工 s: 0 s

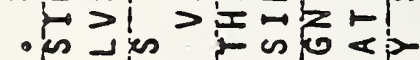

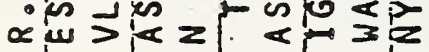

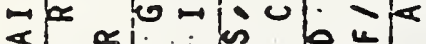
a w w a

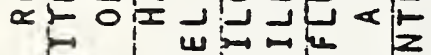

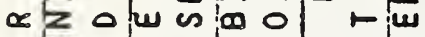

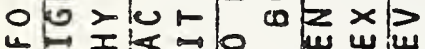
a $a z=2$ on $z$ 皮 o

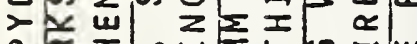
a $x$ I. I 3 os is 0 in

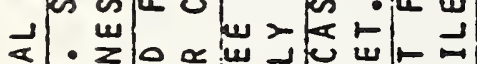
$>0 \leadsto \omega$ w $\sum z$ z

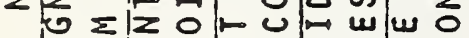

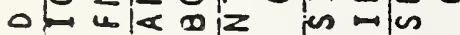
w他 造 光皮

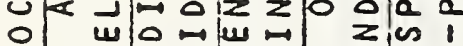

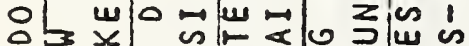

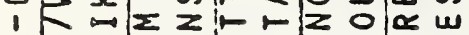

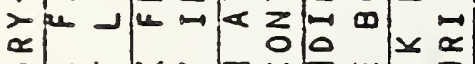
○以

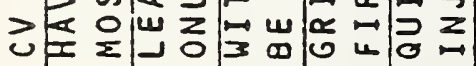

a

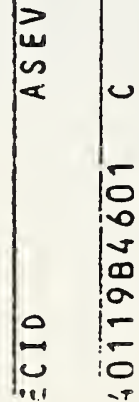

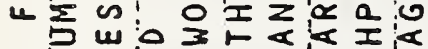

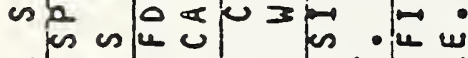

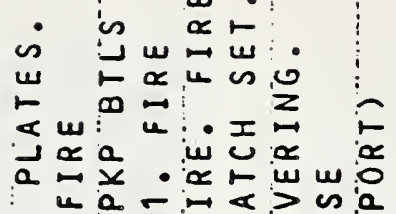
ㄴ. 150 ! 3 언오 는잉 w

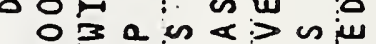
$>\propto$ i. $\rightarrow$ in $\rightarrow$ a $\alpha$

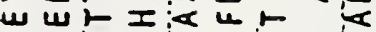
$\downarrow \alpha$ 的山 سأ سن 《Uम

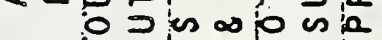

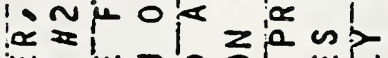
w

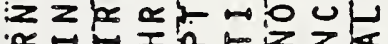
: จั

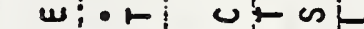
$\propto v<w$ o

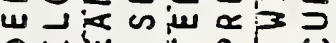
에 w t a

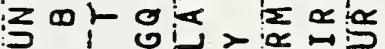

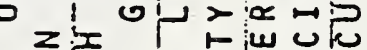
$\Sigma=x$ 눙 이인 $w n \geq \infty z 5$

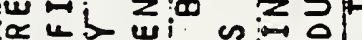
$\omega$ - $n<i \geq 0$ $u=1$ in in 0 m

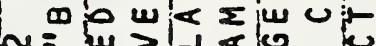
$N=\omega>\omega<0$ 心

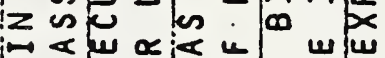
س 可的 us u

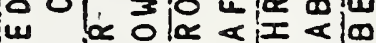

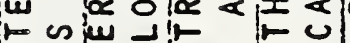

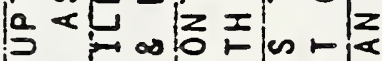

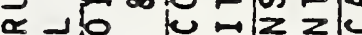
w

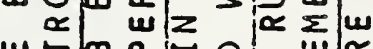

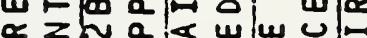

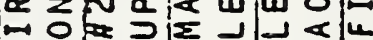
$\leadsto$ 이

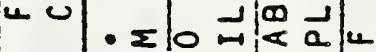
$<z$ u it uk ü

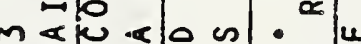

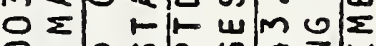
o b esla vorit

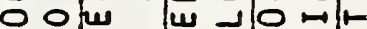

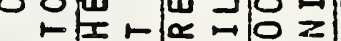

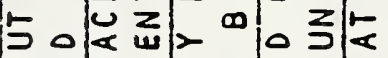
0 - 0 - 0 . a a $<$ 皮 < w $<$ \&

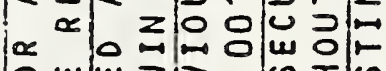
○ w w 30 as x w

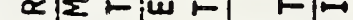
$z=\Sigma \quad \propto ⿻ 上 丨$ o $u=\infty$ la

$\frac{a}{2}$

0
$\tilde{0}$
0
$\sim$
$\simeq$
แ. n:

\& 0 w: w simoin

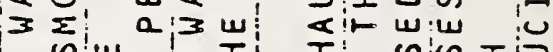
- ñum

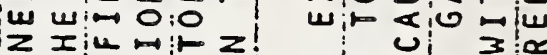

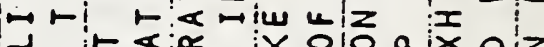

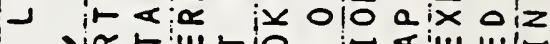

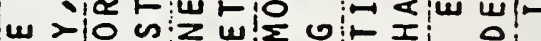

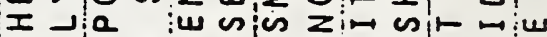
F n $z$ z $z$ oํ

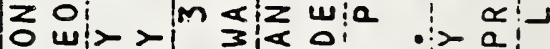

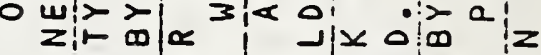
o ख ०ю0 z

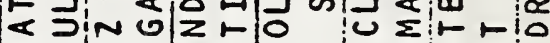

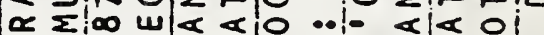
$w$ wio $m$ o ل

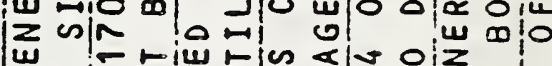
जमF 战

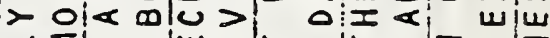
$\equiv \sum$ w

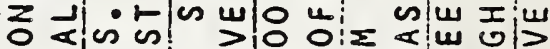
U ग)

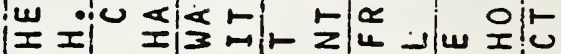

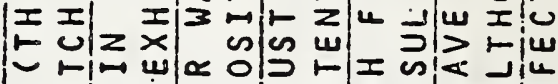

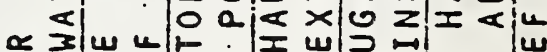

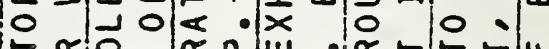
《

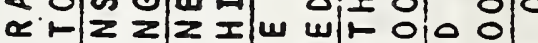
w

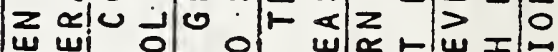

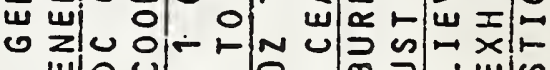
- ul 0 c

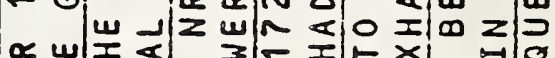
$\approx=10 \times 10$ $z$ I

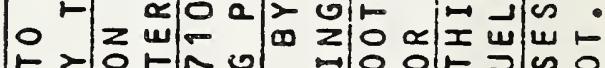

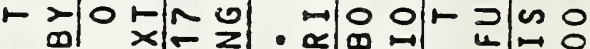

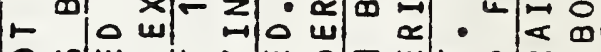

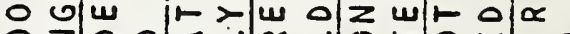

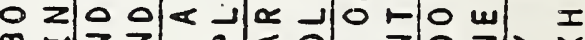

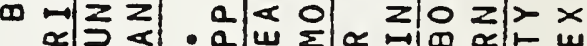

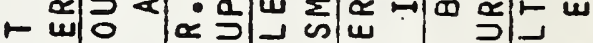

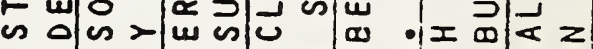

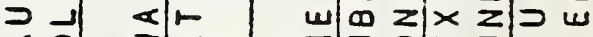

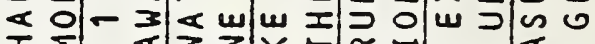

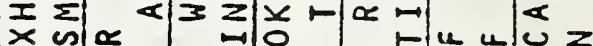

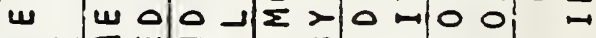
山

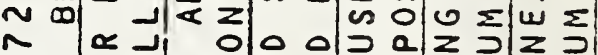

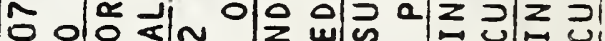
N $\vdash$ u v

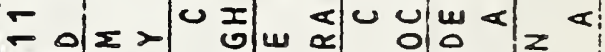
$\bar{m}$ 일 가 $\propto ⿻ 上 丨$ ᄂ

0
-
0
$m$
0
0
$m$
$n$
0 


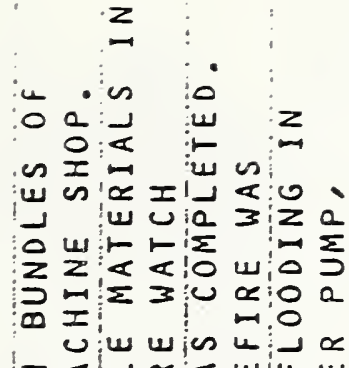

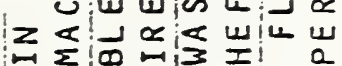
u

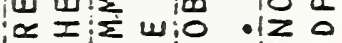
ニ

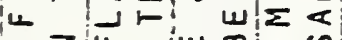

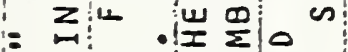

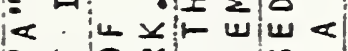
$=\vdash 10$ a en « w in du to mu

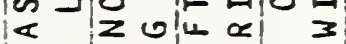
$\rightarrow>w z<w n$ U1m

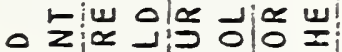

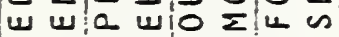
in < wा w $w<$ a U> z 0 ! 2 : o zio zia oit u In $=$ 任zio wil a w w ज

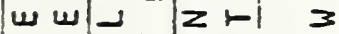

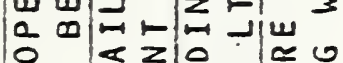
$0 \quad \leqslant z 00$ 0 0

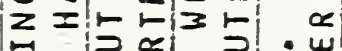
il J ज $a$ I

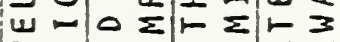
3 I 3ir Ulu 2130 $\sum$ in 0 w ○ ᄂ w

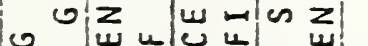
< w w

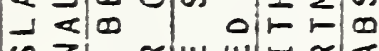

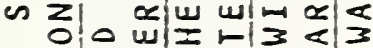

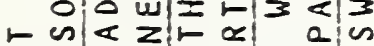
$0 \propto 1=\alpha$ < $<$ ह

$=w=0 \mid=$ w < 0 w wo wir

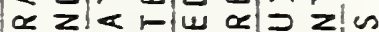

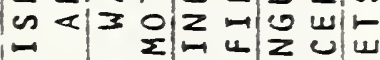
w s

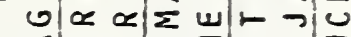

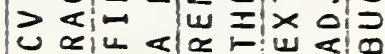

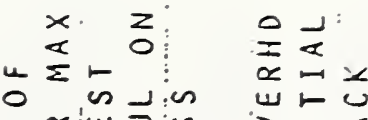
a!w

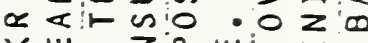
西之: - 0 क

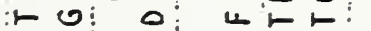
$\alpha x z$ w w : w:

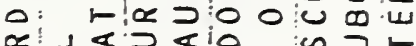
$\propto:-<$ :

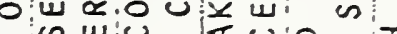
in w u vi: zim - $z \models \infty$ ○

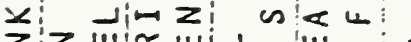

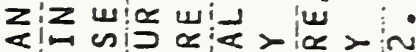

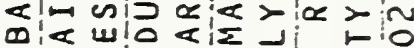
$\sum m$ a o o - a w $z$ u <!o $0^{\circ}<1$ w oir Ilu If a j Jio diz F片 <

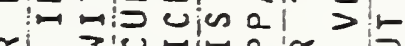

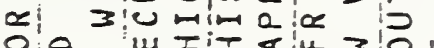

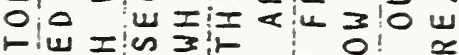
nit

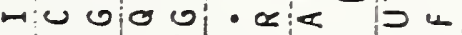

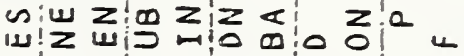

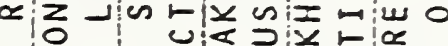

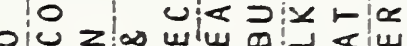

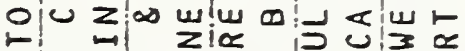
la 0 z

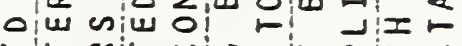
citait vios itajus

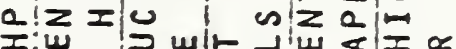

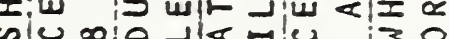

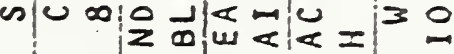
20.0 रा I

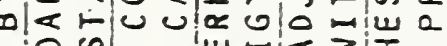
i० का

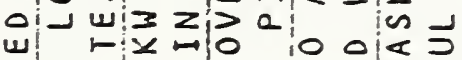
a 10 ujt was

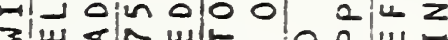
in in $^{2}$ w z出

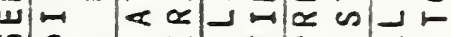

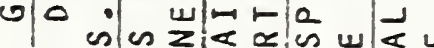
Uu va wito 0 ए जी० खा

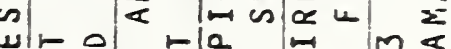
wien o ra

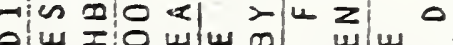

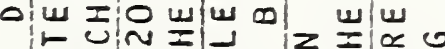
s) 2:0 $\Rightarrow$ uा win $3=$

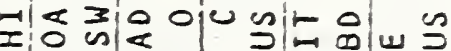

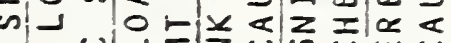
.1- $-\sum^{2} \sum_{\alpha} U$ $\Sigma 0-z \quad \infty$ vा mा

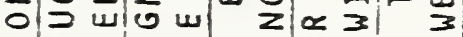

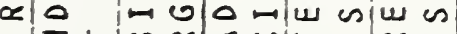
z $-\infty \propto ⿻ 上 丨$

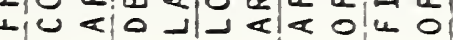

a

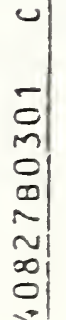

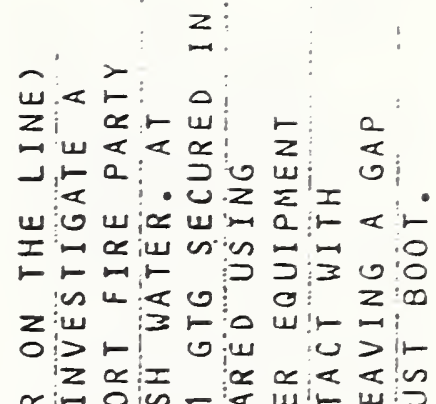
인

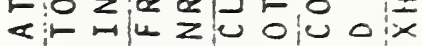
\& w $\begin{array}{llll} & > & 0 & 0\end{array}$

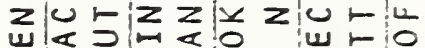

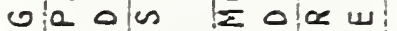
in $D$ o in zin un

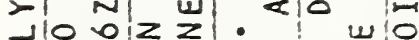

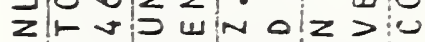
or m ज a : w w

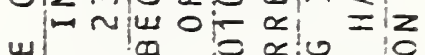
I戈 o: o

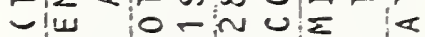
- I - lom ol on

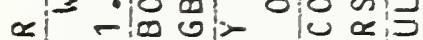
○!

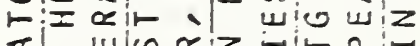
< wles $\propto ⿻ 上 丨$

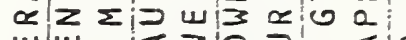
wiv $\alpha \leq 030$ w $30 \times 0$ 2 $20=0$ ง| 4 w 0 mic 0 - س w w w Ir $z \geq$ s 0 in

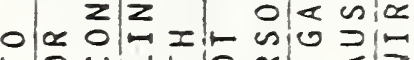
느의

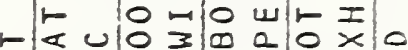
⺊造 이 o

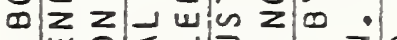
w 0 伐 w $z$ la $\geqslant>0$.

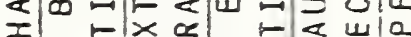

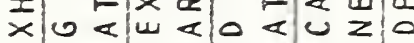
w $z u$ a $z$ j $z$

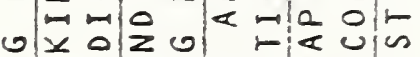

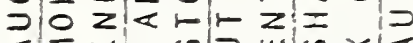
< $\sum_{0}$ 0.

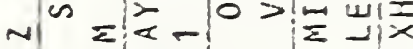
no 0 ?

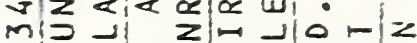

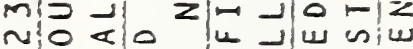
NU UN

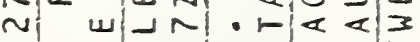

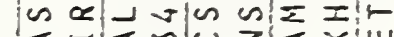

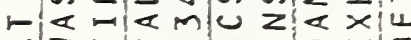

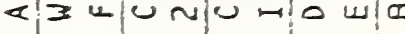


$\check{r}$

๘

0

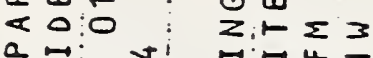

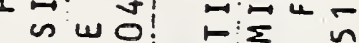

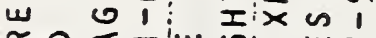

व $0: \ll-$ ज

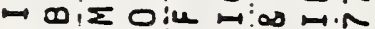

$4 \vdash \ll$ ए

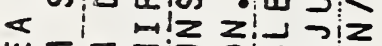

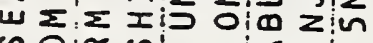

थ 0 ic n $n$

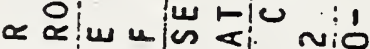

U OIY OID oiv-Im

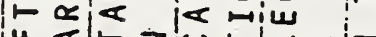

u $<1 \vdash z$ z

$<\geq \mid z-1$ का

or

u ⺊

I

i

in

$-x<x$. oiw ol

in J $0<10$ z 2 alu

$103: 0 \leqslant 0$ z

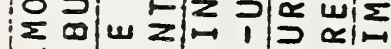

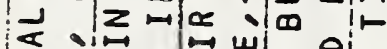

wiv u:3 vix wies

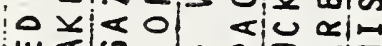

:

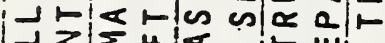

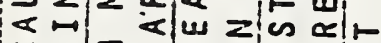

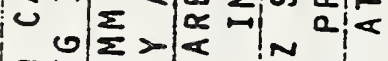

$0 r<x$,

o $0 / 0$ F,

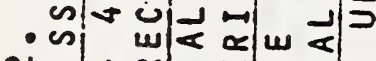

in $N a$ a

2 0 0|⿰

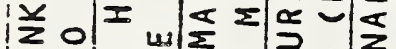

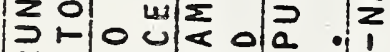

$\propto \quad J$ < 0 w win

- UU

$w<\frac{0}{\alpha}=m$

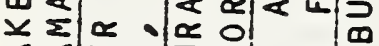

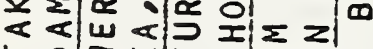

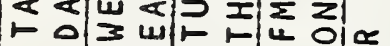

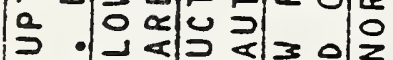

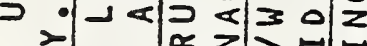

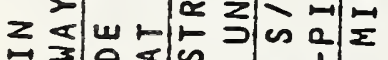

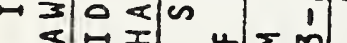

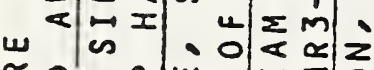

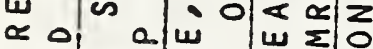

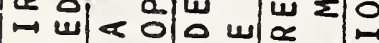

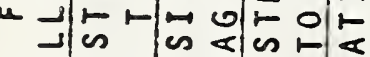

i v 0 w

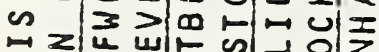

$\rightarrow z$ u w|F

उ w $0,5 \infty$

$>\quad x \quad 0 \quad u$ a

0 $>$ z $\propto w$

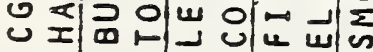

0
4
0
0
0
0

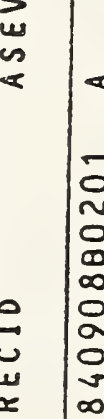

a

$\sum$

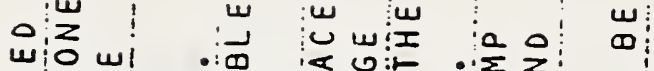
Ju Iio w:《 : a:

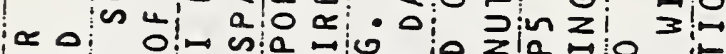
$0 \leq 1.0$ 细

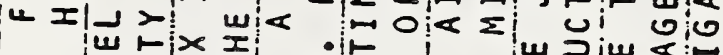

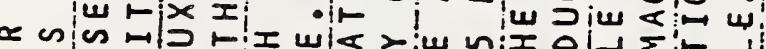

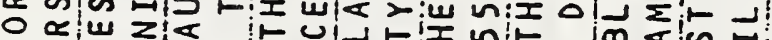

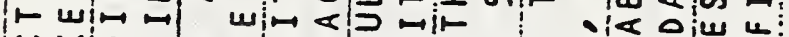

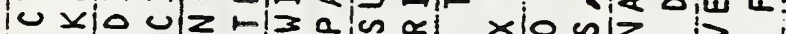

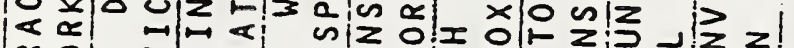

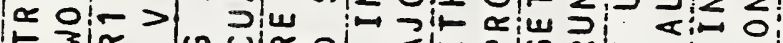

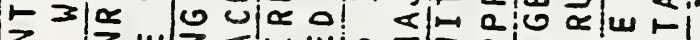

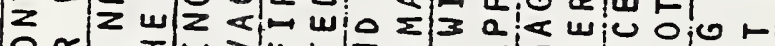

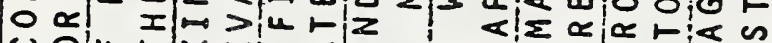

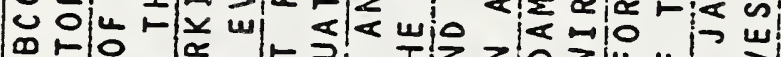

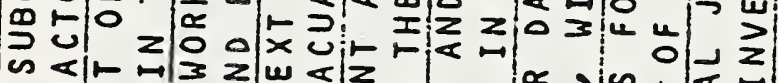

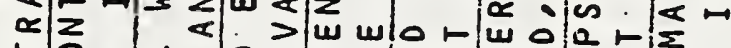

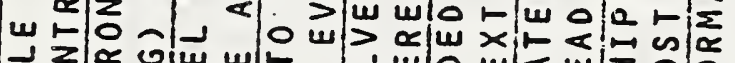

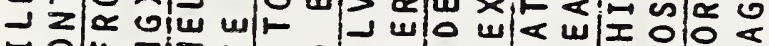

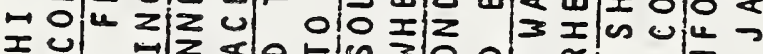
I

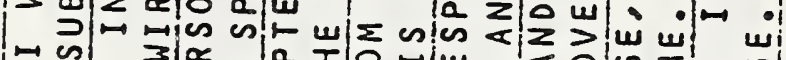

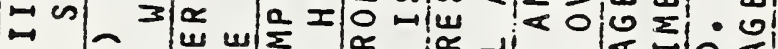

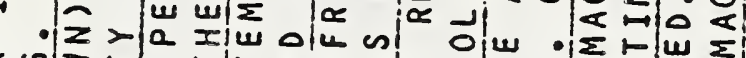

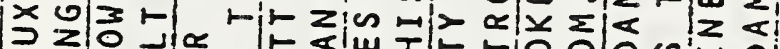

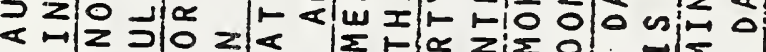

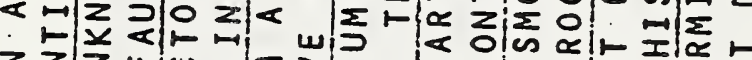

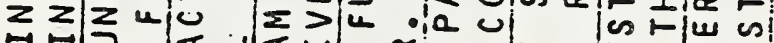

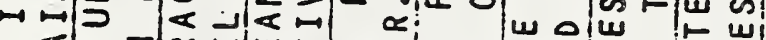
<

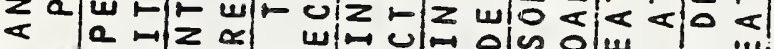

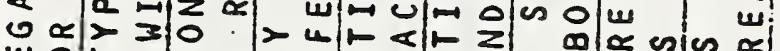

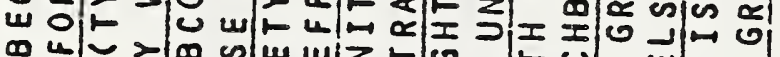

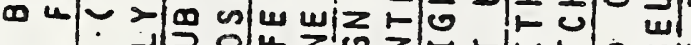

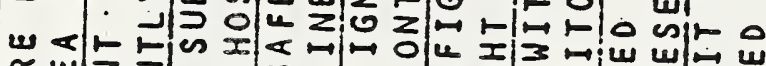

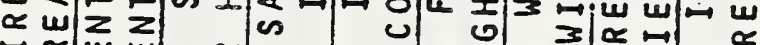

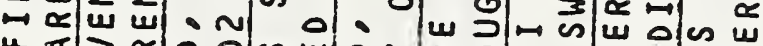

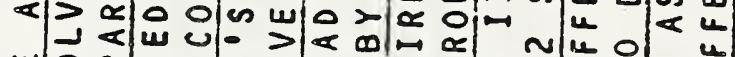

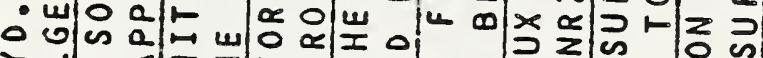

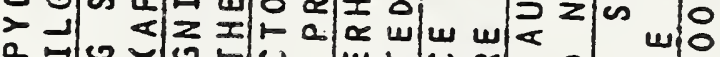
을

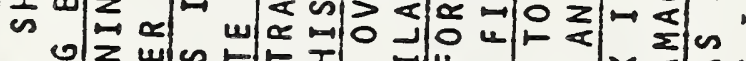

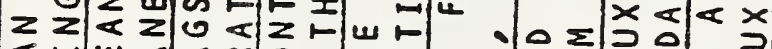

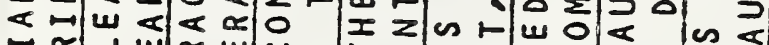

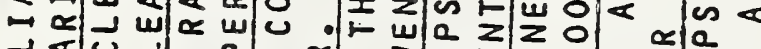

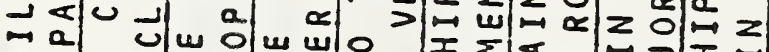

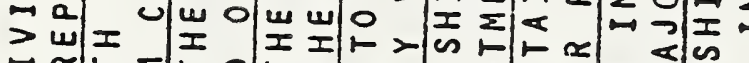

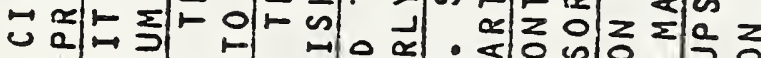

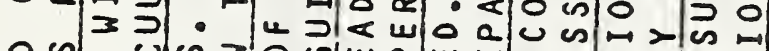

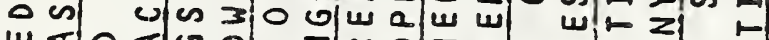

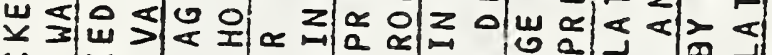

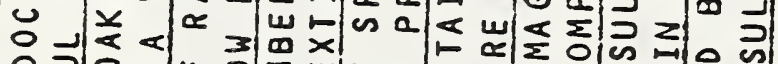

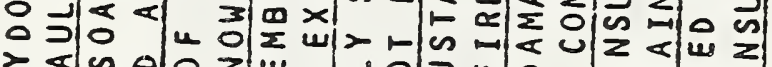

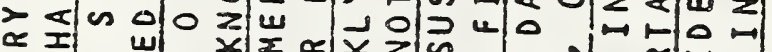
c I แ 0 a

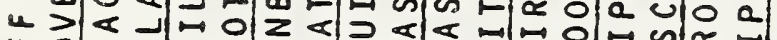

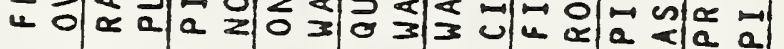

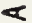

10

$\frac{a}{\Sigma}$ o 0 ir im 0 . rizo: a n <i $0: \propto<i 0 \ll$ $z: \omega \propto ⿻$ o mi $\sum_{i}= \pm i z-i u$ 少 $\infty$ in w 仰 wizvirai

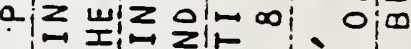

-

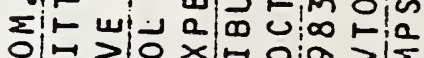
ol $\sum$ oाu का० . > w wo niz dis wir का w ๙

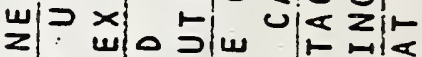
$z: z$ u In

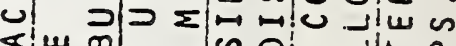

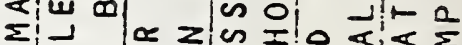

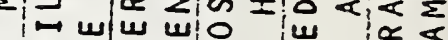

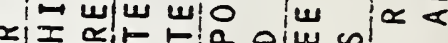
II cit

0 उ

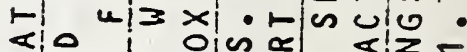

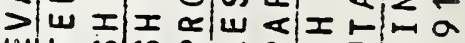
U. 1) w

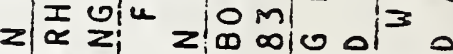

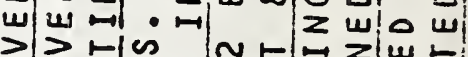

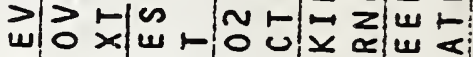

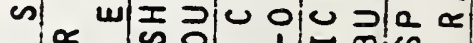

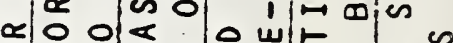

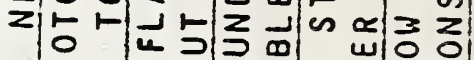

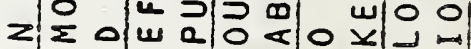

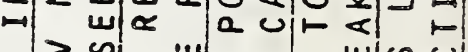

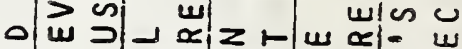

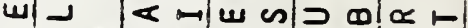
$\vdash$ U 당

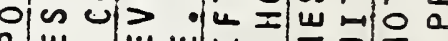
난 $u$ w 卌尗 Uト w $\overrightarrow{0}$ ol $m \sum a \mid \supset$ uir a $a$ o

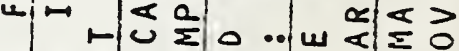

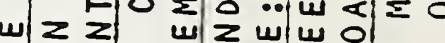

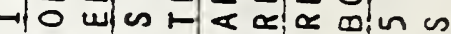

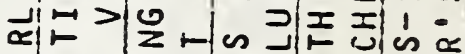
$\varangle \leq \sum \backsim z \propto ⿻ 上 丨$

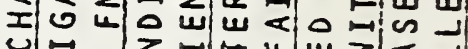

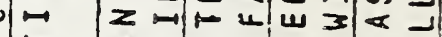

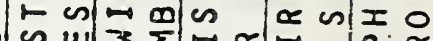

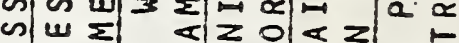

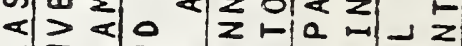

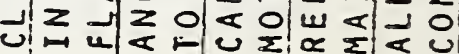


ind im

in $x$ o:

Jien $4 \alpha$ :

w $<x>$ w

w $0 !-1] 2$ s

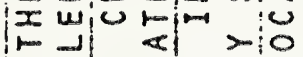

$>-\frac{1}{2}=0$

क ज ज $>$ का

$<1 . z 1$.

0 in $-001 z$

us slu U|w

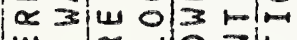

w $\propto$,

उ)

$\omega \alpha 10 z \alpha$

क स IIU mo

wa

w 0 I

es $\propto$

$3 x$

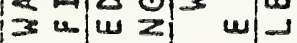

Jin as a

int esiles max

$\propto z \ll \supset \propto$ u $]$

u $<$ v 20 o

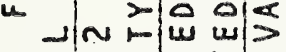

$=\alpha 0$ a 0 L

$<\propto \mid 0<10$ \&

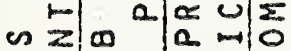

in w w

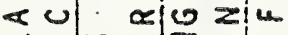

d $12 n=0$

- o $x z$ z

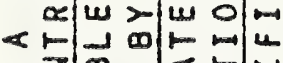

$z$ का

인요 인

- $\propto$ I 0 -

- m

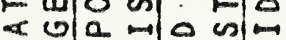

$u x: \Rightarrow \geq w$

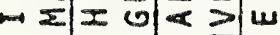

$0 \varangle r z \quad z$

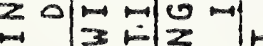

ㅇ $x=150$

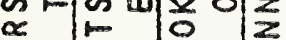

w wa ol

$\infty$ a 2015

$\Sigma<w$ \&

w $\Sigma\left[\begin{array}{lll}0 & 5 \\ 5 & 0\end{array}\right.$

0 心 $<$ w $z$ w.

$z \propto \quad z \quad w \alpha$

$\because 3-7 \sum 0$

3 a $x$ in $\alpha$

$0 \mapsto-\infty$ of $\alpha$ o

$\pm \propto-z<$ w $\alpha$ a

0 일 연

o win

$z a$ in a w o

* : x wा w

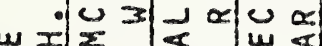

$x y=m \leqslant$ ma

or $\alpha$ o क क w

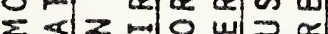

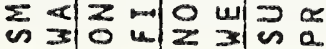

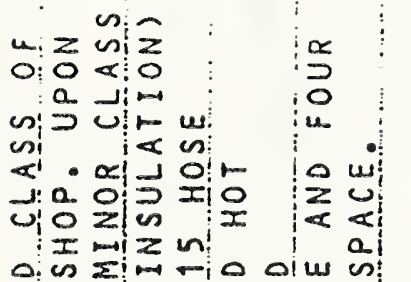

$\sum_{m}^{\infty} \quad z_{m i} z_{0}$

a) $0 .>-$

w: $z$

W

उ。

u -1 u

$\Delta \ln _{4} \hat{\sim} \bar{x}$

0 is $\sum$ -

出 $\propto<\alpha$ ol

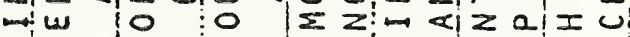

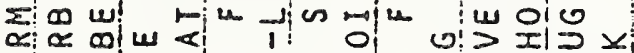

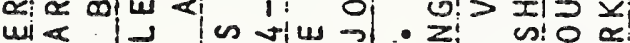

$\mapsto \infty$ o

w

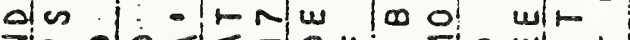

当之 에 w

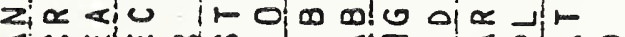

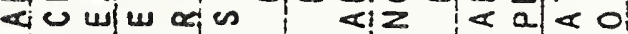
a a of to I

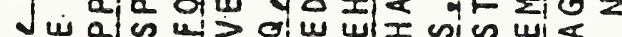
ni $0<0 \geq 1 \propto \alpha$ a $a$ 에 कn N N

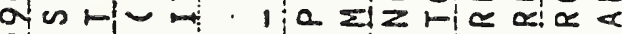

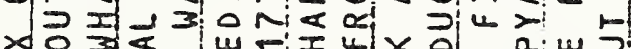

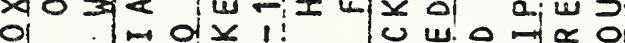

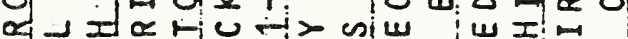
先 ân

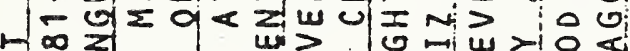

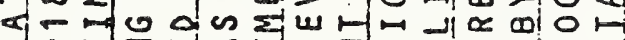

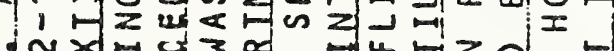

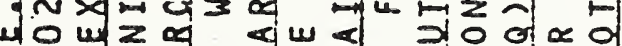

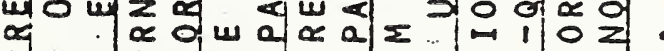

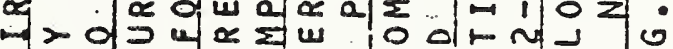
나 < 에 3 का u जu - w

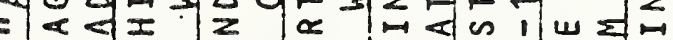
का

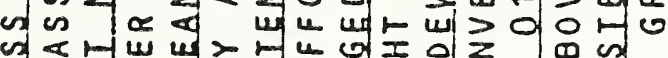

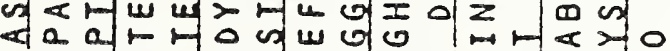

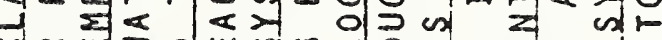

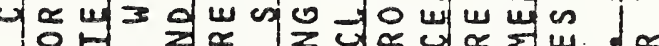

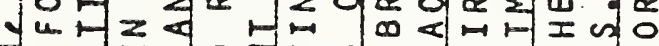
गै वu 0 z d> I

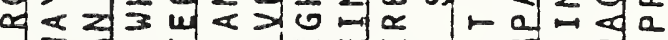

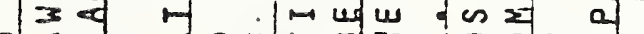

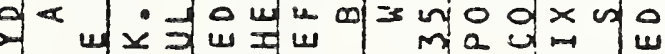

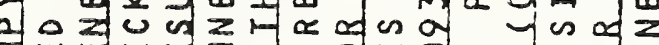
$x$ ш 내 w $z$ 의

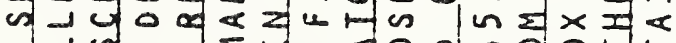

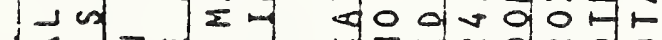
西出

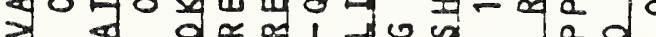
स n 4 w

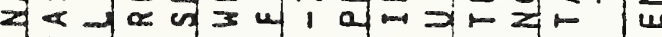

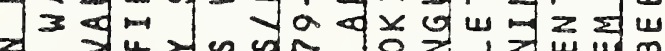

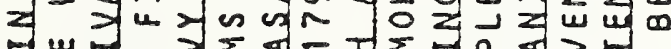

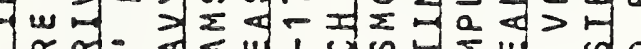

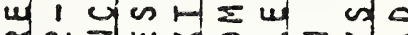

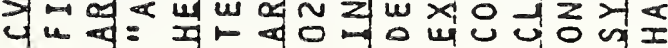

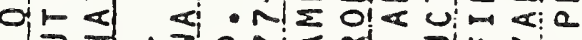

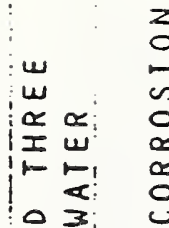

12

$z$

w! ब

os $z$

I $₫$ or

世综

$\sum_{z}^{\alpha} 0^{\alpha} 0^{2}$

u文

0 $<$

$\sum_{m \rightarrow 0} \omega_{1}$

m

$<$ a t

$\propto<<0$

a 긴

in o

- $\omega$

w w $>0$

w

迥了顸

1上 $<$ a

옥인누

$\propto<\dot{\alpha}$

-

1 s

zo

$\supset$ 의

0 a $u z^{2}$

의 $\omega$

$z z 3$

0 :

tisus us

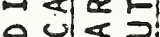

$z 0 z$

U

म0

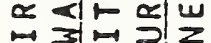

《3 3 ol

- 自的 4

에: 2

의 3

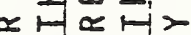

呦号山

$\propto 3 心 35$

o w us of

u of n 네응

w

떠. 거

- I 5

-

2 的俋。

क्षे के

a

0
5
0
0
0
$n$
0
0 


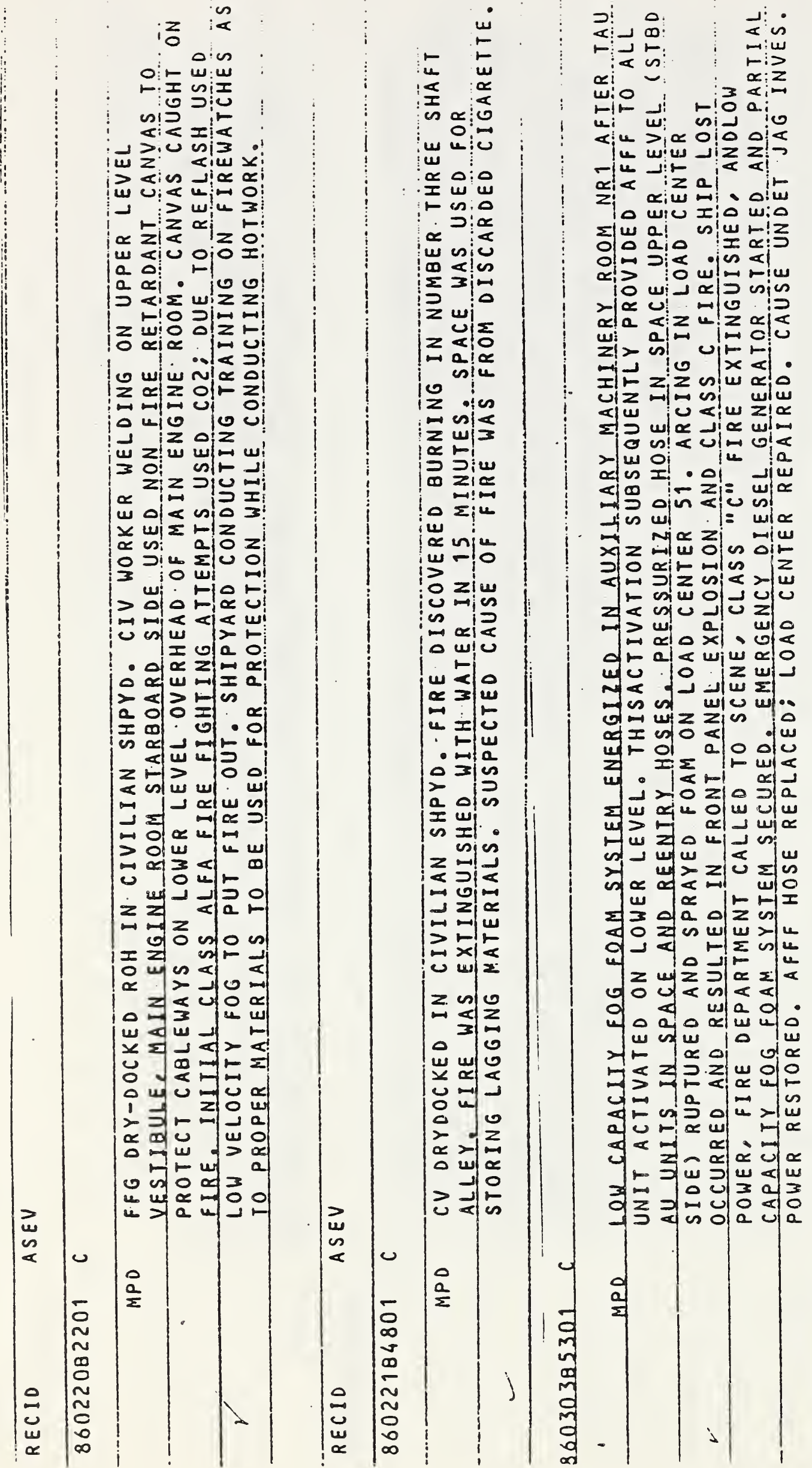

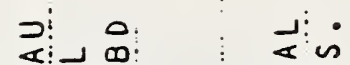

的: 1

wio

ui w जi

$\ll u>\propto 0$ o 0 :

- u w! ए ए

o⿺ izal oir

o wiu Iio wio

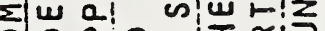

음

(

( 10 a

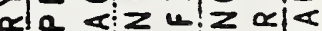

w: a.m

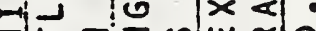

$z \geq \sin \propto 0$

×u $u<w z l a$

$\Sigma \geqslant$ wa d w 의

सा $-z=\omega \propto$ $\rightarrow$ की

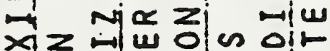

$\supset 0$ ar

ט.

《 क ज य

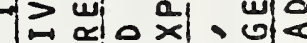

or $a<w_{i} w$ o 0

U

$n_{n}=\geq 0$

I

는 인 인

$4: 1<510$ a

피 4 ol uी

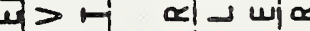

N $\omega w \ll$

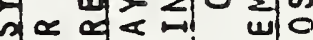

u $\propto$ | $⺊ 尸-1$

$\Rightarrow$ a $a$ ol $z$ in

in w 4

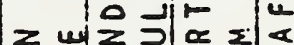

我

u 0 in 40

u

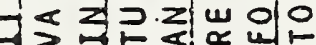

늠

$\propto \propto u$

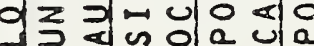

$\mid$

ᄋ

$\simeq$ nz $z<0: \checkmark z: 0 z$

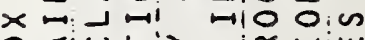

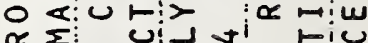

a v zu w

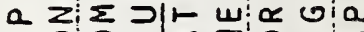

< 0, 3《 2iones

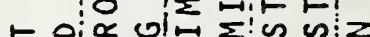

$<z ! w z \mid x \propto$ w $w$

J $\propto$ mo w $>\omega$

. o!

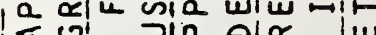

活 0 긴 일

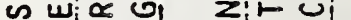

$\rightarrow$ s $<w 0$ ola z n

₹ 3 olw

되 $\propto$ 무 जा

잉 z w \& क

แ

1 에 2 w이

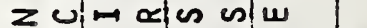

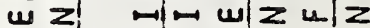
$\supset w_{1} \propto u_{1} 0>003$

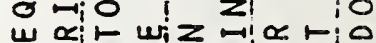

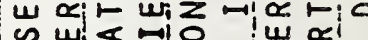

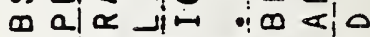

$\supset$ xo व

का w> बर 1 is

II 10 o 2 is

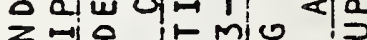

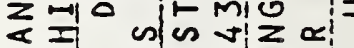

कीu क्या 1 - $<4>\ln 20$

I 서

0 为 1 -

जo o 0 o

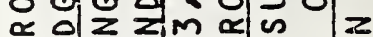

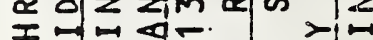

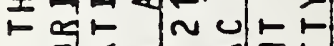

ه $\ll-7-<z \vdash u$

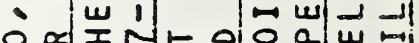

$1-\alpha \rightarrow \ll \alpha \rightarrow$ in

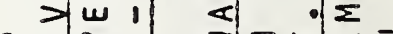

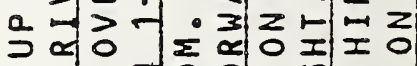

$0>>$ 경ㅇㅇㅇㅇㅢ

( $>$ r

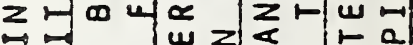

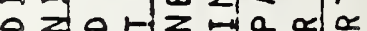

《山

世

\lrcorner - $0>2$ ज $<\omega$

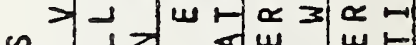

n 0 긍

는

w 간

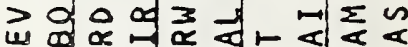

म장 जी

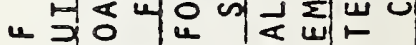

○ व

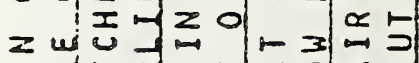
$\because f 50$ 눙 \& क o $\times$ w $\rightarrow$ a 0 도 3 고에 $z w \omega x$ U 코 क

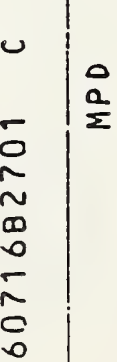


o:

Ziu

- $x$ w u w 0 > 000 w $\alpha$ 1 $0: 1$ -

o a a u⿺ 万!

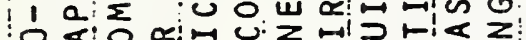

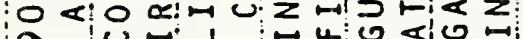
a

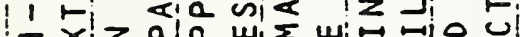
-

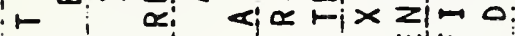
a w

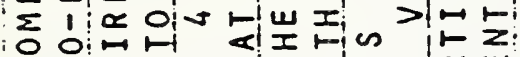
U I:L III I

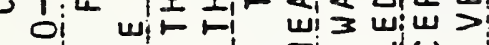

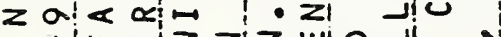
in 1 in $-3 z$ z $z$ w w व $\mapsto$ a: 0 Z u $\sum_{0}=1$.

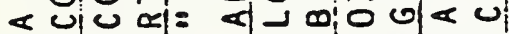
in oñ. za

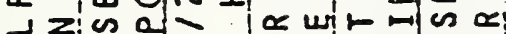

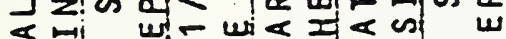
$<-10$ o u $w$ o $\varangle \mapsto \propto$ u. ₹u. in a u -

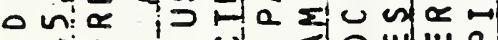
wi ज ज द $\propto \sim i m$. w

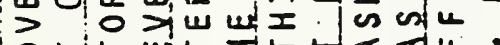
$0 \mapsto \vdash$ แ

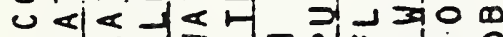

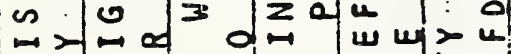

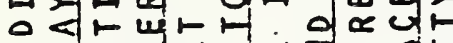

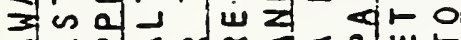

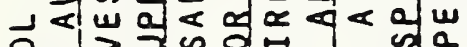
ol 29 I

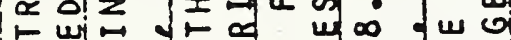

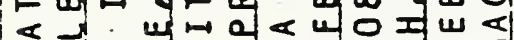

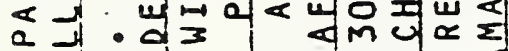

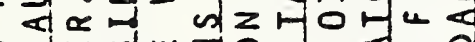

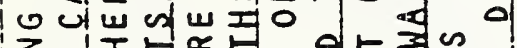

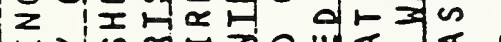

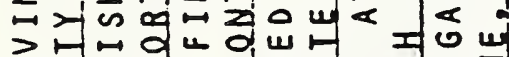
舟 0

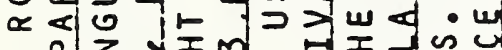

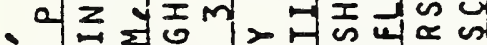
مu

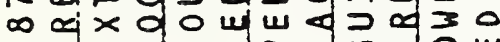
$>$ u o $⿻$ \%

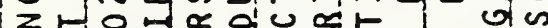
ฯ वे maI $I$ ए

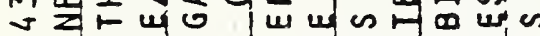
$\sim \mapsto \mapsto 1 \mapsto-$ a $<\&$ 의

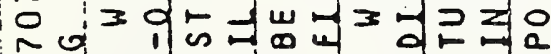

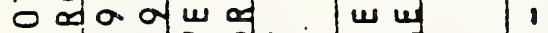

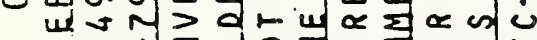

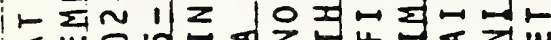
<u

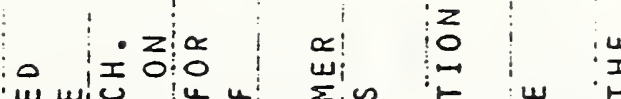

: w wiv

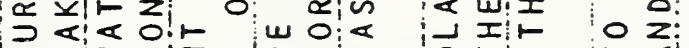

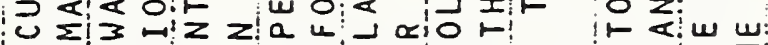

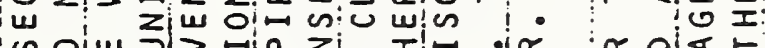

u

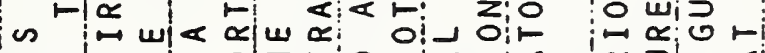

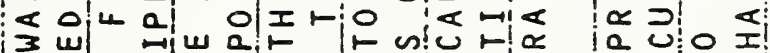

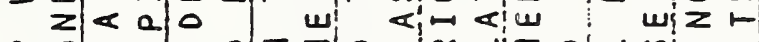

a 0 a

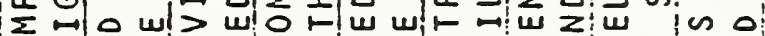

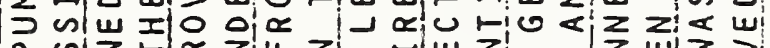

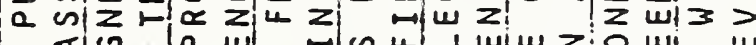

u

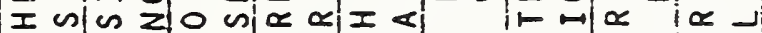

I 10 (ग)

उ.

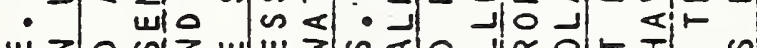

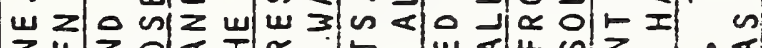

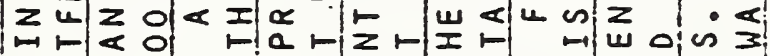

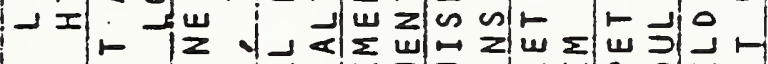

ш z $\backsim u$ of

$0<I 0, \omega D$,

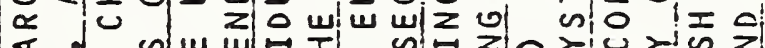

I

ज w a w

थ

月

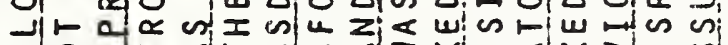

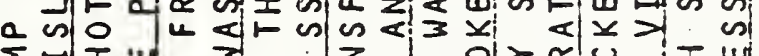

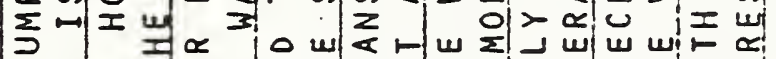

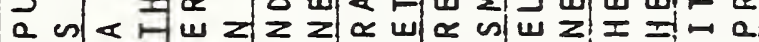

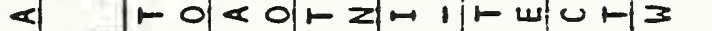

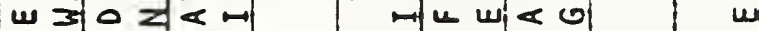

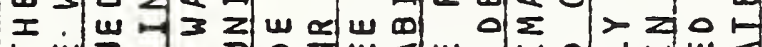

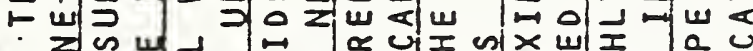

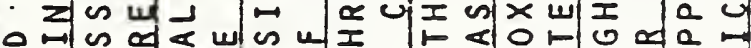

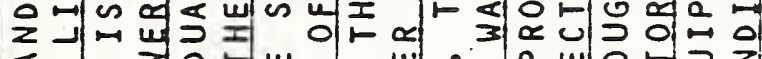

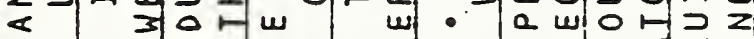

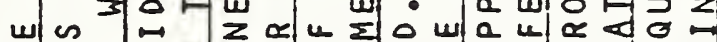

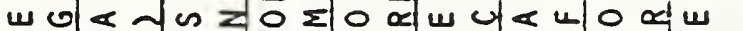

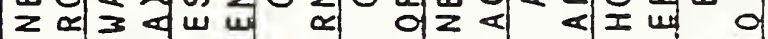

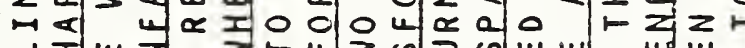

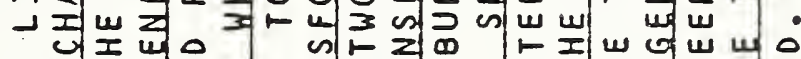

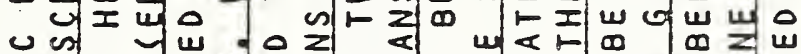

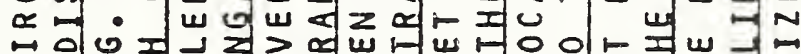

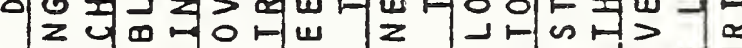

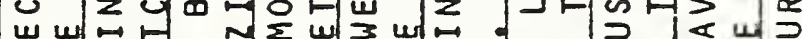

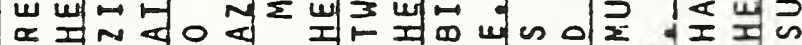
폄

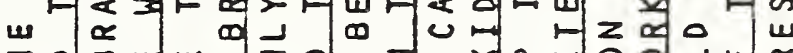

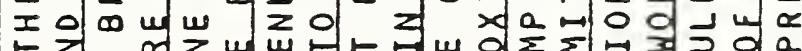
$F$ 인 u

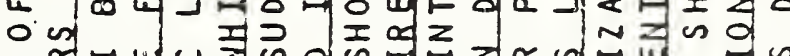

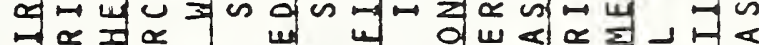
Z

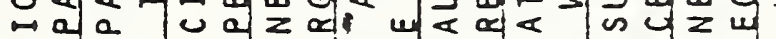

zuш 의

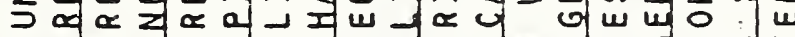

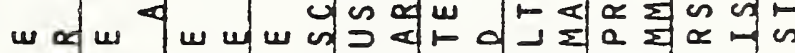
얻 


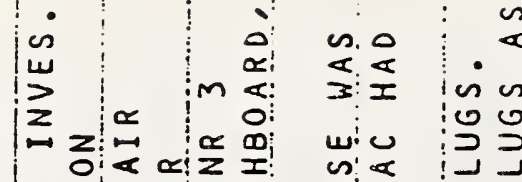

o a w

1의

in «0 0.1 tius

(

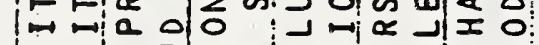

o zhma u w

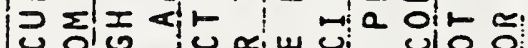

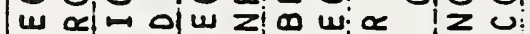
क ज I wi w o z z $u_{n=1}^{\infty}$ en

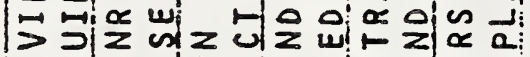
이: $\alpha$ n. en nono oft

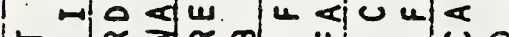

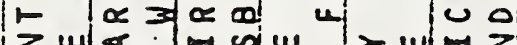

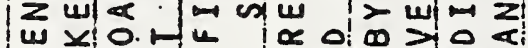

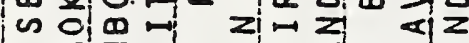

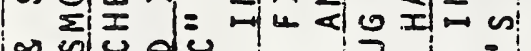
-

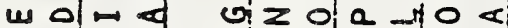

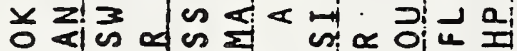

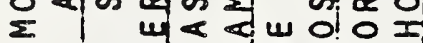

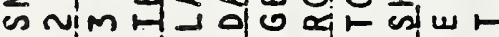

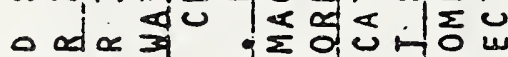

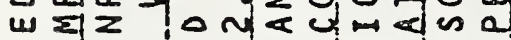
a $4 \geq 00$ : 0 I n $\Rightarrow$ o वरु wi Hoz

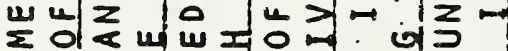

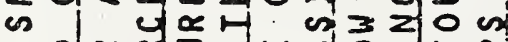
olv 역 w जो

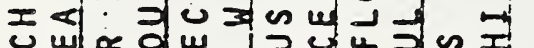

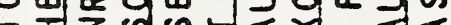
政

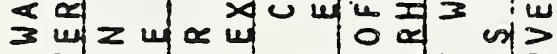
깅 $w$; d w - O $3-1, \alpha \geq n$

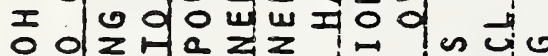

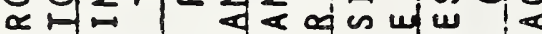
$z=0 w$ o a $20 \geqslant$ w

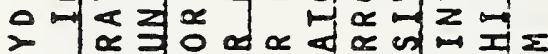

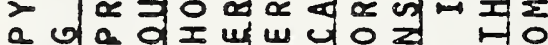
ㅊㄱㄱ क ㄸ

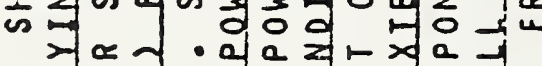

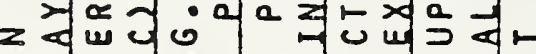
$<0 \leftarrow z$ o 0 w

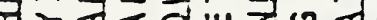

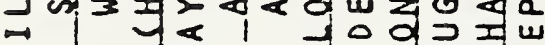
$\rightarrow \infty 0$ a 0 w $\rightarrow \mu z$ or a $w$ o of $a$

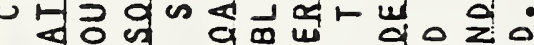

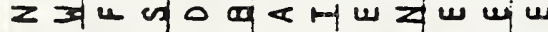
- 니 a zw a

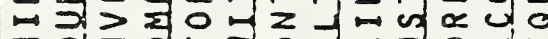

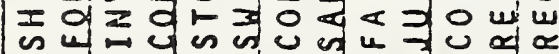

io

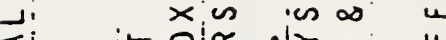

sir

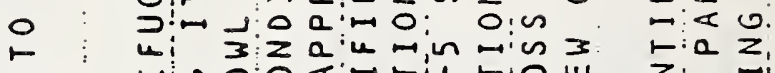

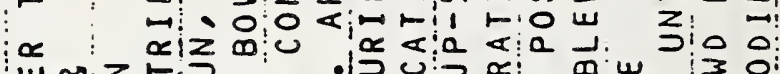

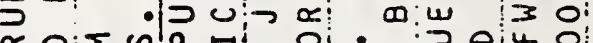

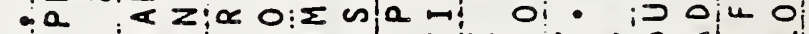

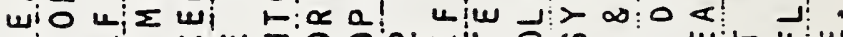

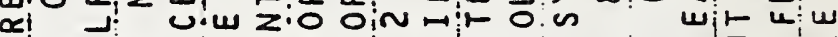
we \& I I

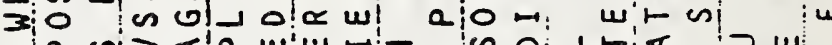

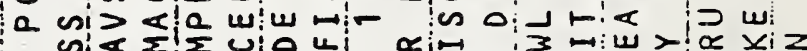

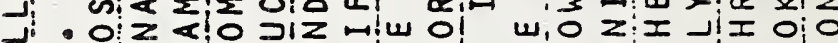

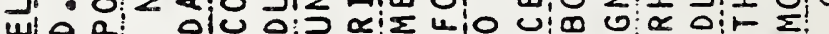
Wu

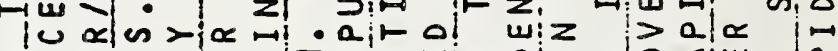
म

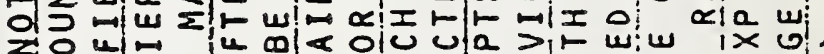

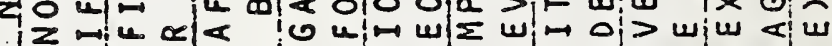

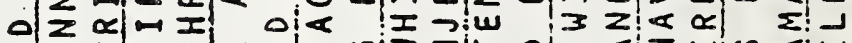

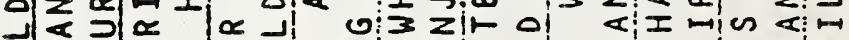

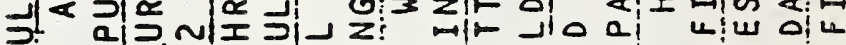

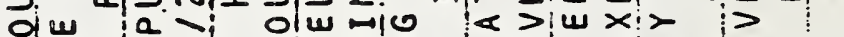
ज次 $\mapsto<n 10$ sil Hu जo

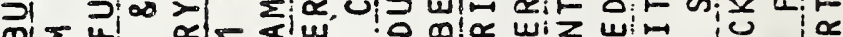
a

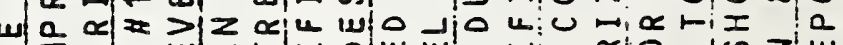

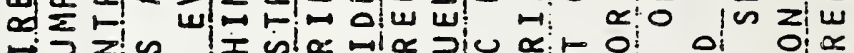

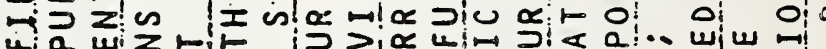

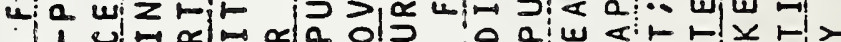

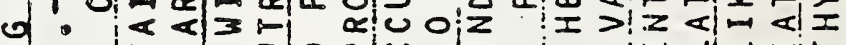

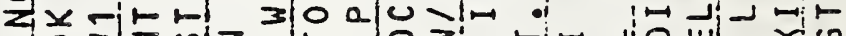

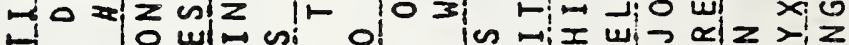

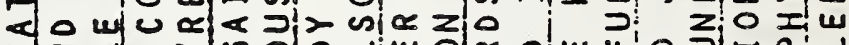

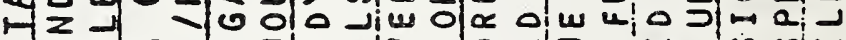
की जु

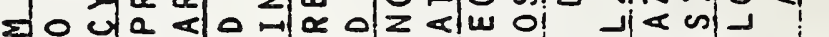
लㄴ

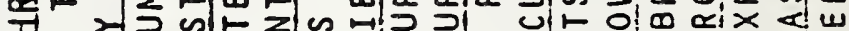

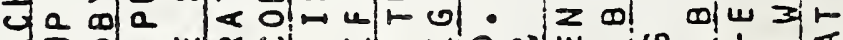

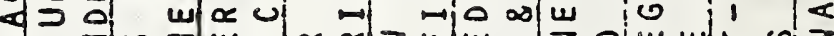

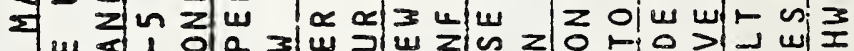
w ख 1 잉 द

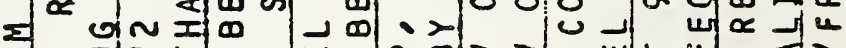

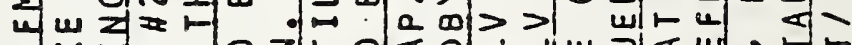

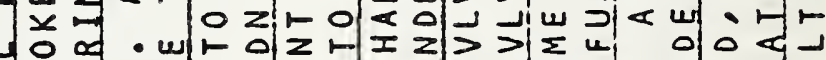
-10 의 $\propto$ 네 बं के

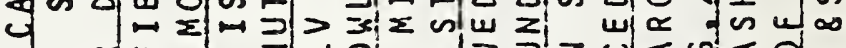

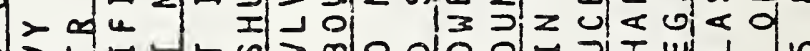

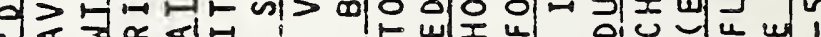
خा

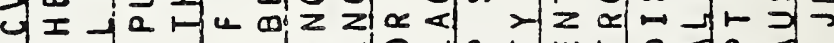

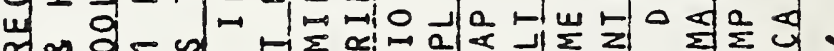

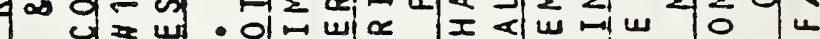

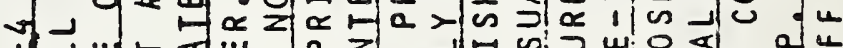
y. 由

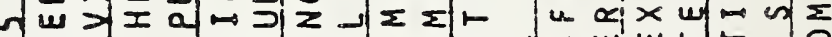

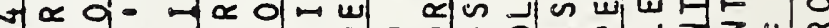

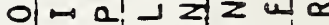




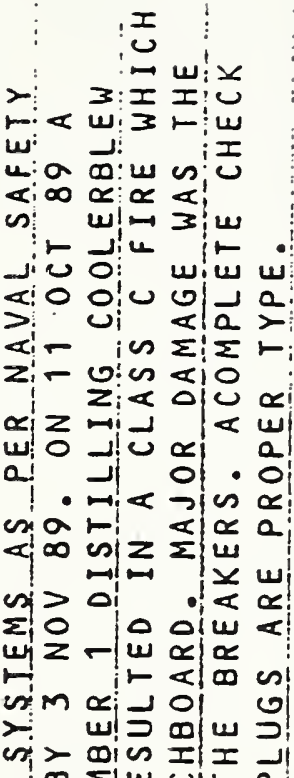
๙ แ ब

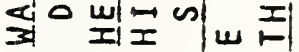
« w 30 क L $\vdash \propto \frac{1}{<} w$ 이인

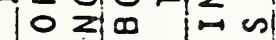
w 겅 강

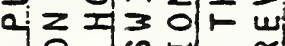
ज・山レ 3r

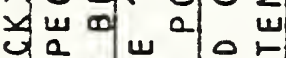

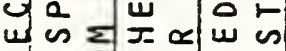
II 2 마네

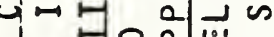

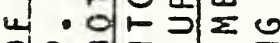
व० $0 \geq z$ «1 แ

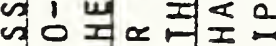
щ $\backsim$ w - 10 d) 주에

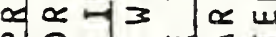
is ज 0 जा का

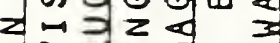
넉코레 वे n\& 4 of

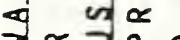

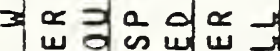
a $r$ का ज्ञ $-205 \geq 0$

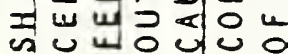

inz I I v

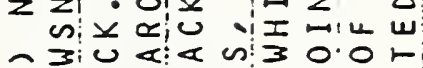

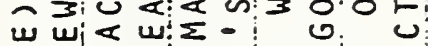

上此越皮的

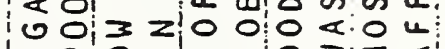

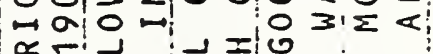

$\simeq-\vec{i}, \overrightarrow{1}=$

u d o

м

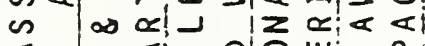

\& सा की का a

miz 닝 a

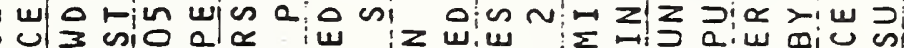

<ا

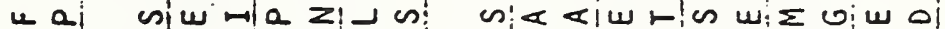

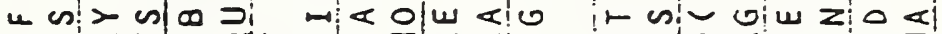

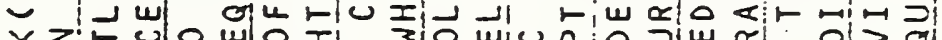

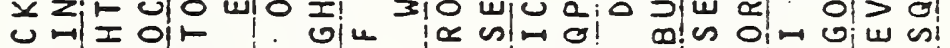
$\leq 0$ of 0 은

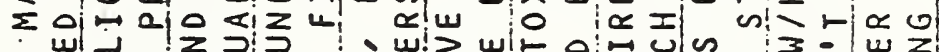

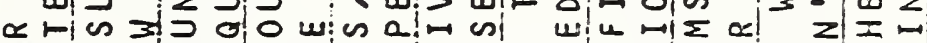

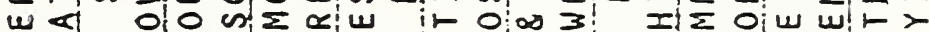
I I

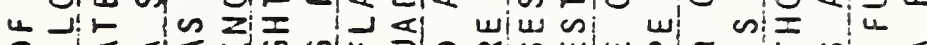
○ «《

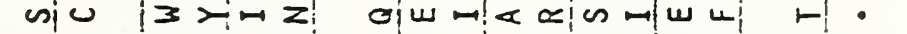
山.

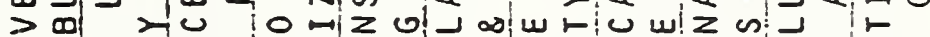

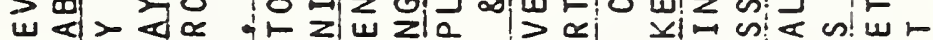
$\downarrow$ 실

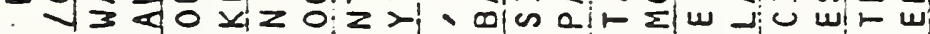
थ 읭 잉 w

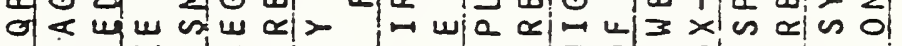

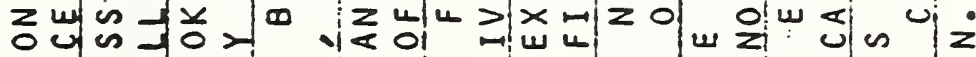

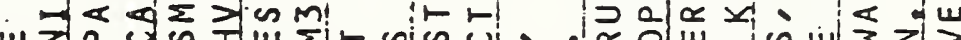

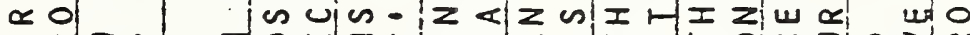

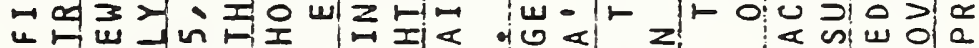

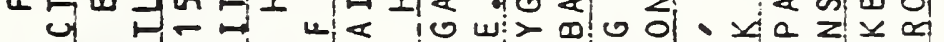

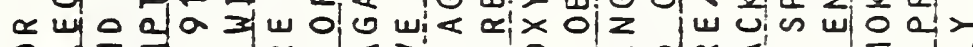

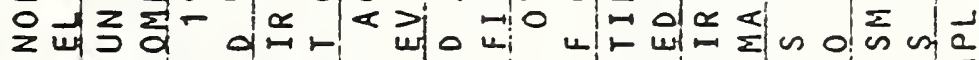
$\rightarrow \quad 0$ वो

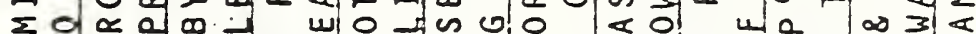

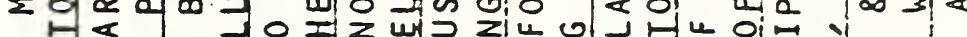

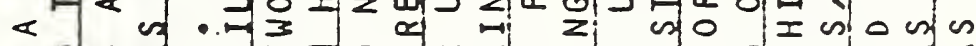

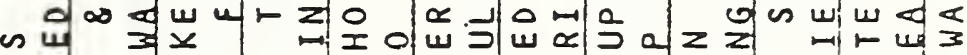

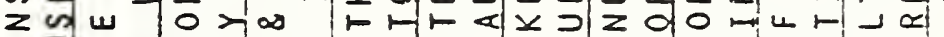

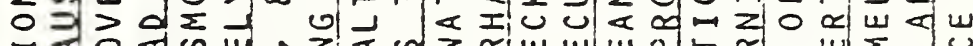

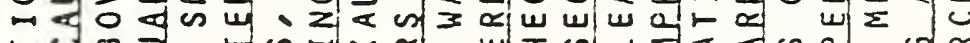

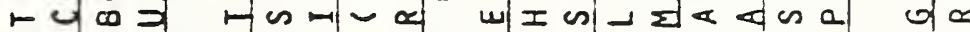

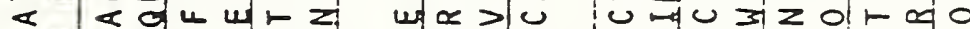

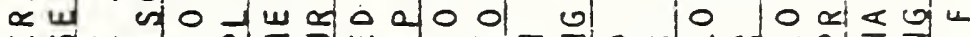

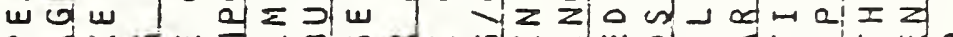
a d ㅂ

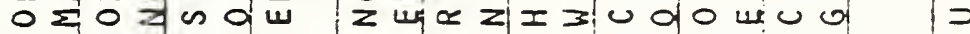

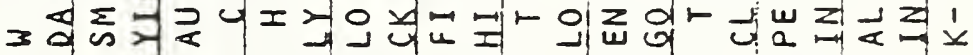

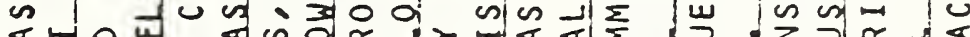

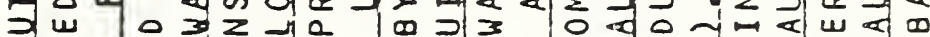
$\sum$ व

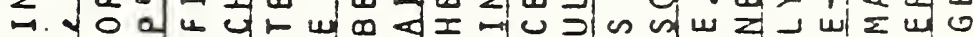

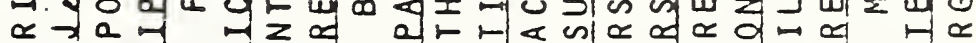

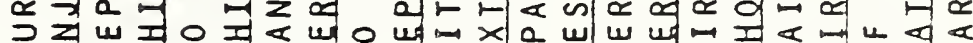

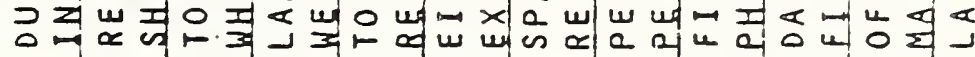




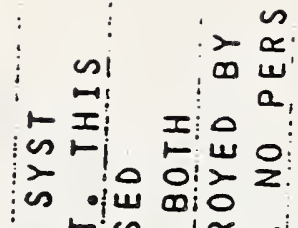

๙

il: 0 il

th 0 al

(

Z

a 200

$\rightarrow \infty \alpha=\alpha$

a \begin{tabular}{l|lll}
2 & 0 & 0
\end{tabular}

- Jiu ul 2

10 in $<0$

↔ वाष

>

( $a$ !

$z m=40 \mathrm{~m}$

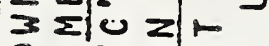

0 cil mis 0 0! 0 z

I UI

es

$\varangle \geq \mapsto \mapsto 0 \propto$

$3 \times 1-1 \geq w$

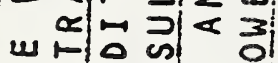

c o w n a :

$\Rightarrow \omega<\propto<$

$\begin{array}{ll}5 & 0\end{array}$

$\propto<1 \geq 0$ : $\Sigma^{\omega}$

w I $m$ को a

₹ a.

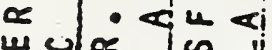

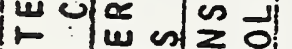

$z>\mid \begin{array}{llll}E & \cdots & < & 0\end{array}$

$\supset$ 드 $I \propto$

O

○ीu

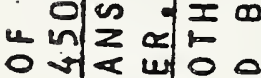

0 - $\leqslant 40$

$0 N \leftarrow$ \&

$z$ I U

$\therefore$ ol $2>5$

$-0,32$

I $\rightarrow$ of ज

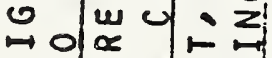

$\rightarrow-2 u$ 긍

- 의 의

$\mu$ of $=0$

$m$ us $=$ a u

$D \mapsto-a$

0 a $>$

$\omega 3$ क o d 1

$\propto<$ व

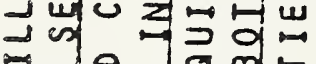

$\alpha$ of $w$ of

○ा

v $\neg w$ u

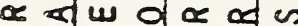

$\infty$ 누 의 $=<$

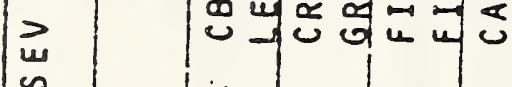

皮

$\propto \frac{\pi}{3}$

$\leftarrow$

य $\infty$

<n!

$1 \times 0$

10

3 o

o t

wa

< $\frac{w}{\alpha}$

$\vec{w} \alpha$

$\propto w$

$\propto \omega$

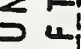

פ

Z $\backsim$

$\sum \sum$

心

D N

足

깅라

¿ wi

$\Sigma$ of $w$

$1-40$

$\sum_{n=1}$

$-90$

$-$

의-

के 에

- 전

w

as of

$\rightarrow \infty$

0 나

0 of

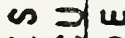

$\rightarrow$ U 0

- un $\overrightarrow{0}$

$4 \sqrt{2}$

$\rightarrow u$

4 다 $I$

$4 \sum_{0}$

$\leq 75$

a g

ad $x$

क ज

$<2$

$\rightarrow$ u 0

is

암 


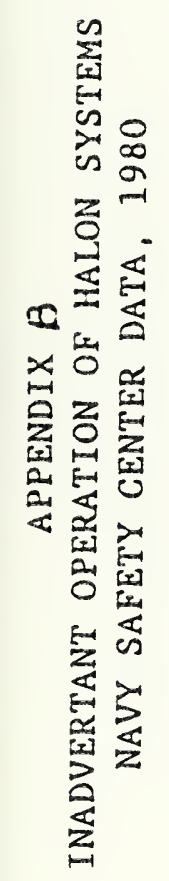

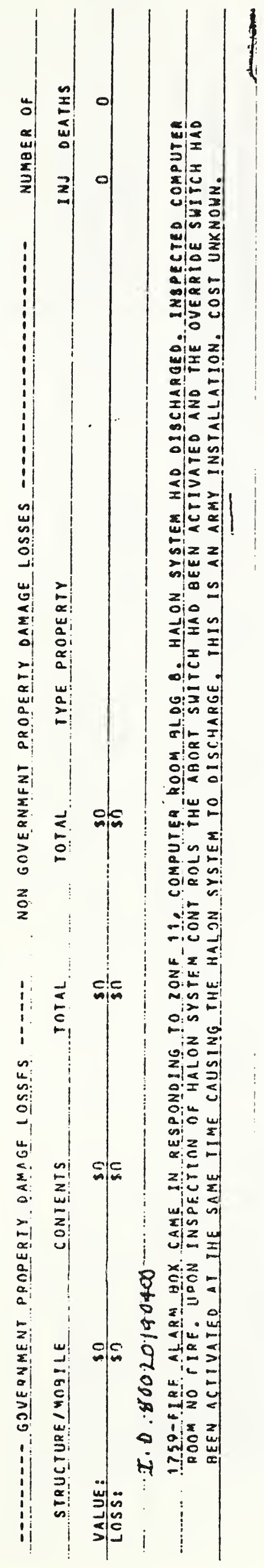




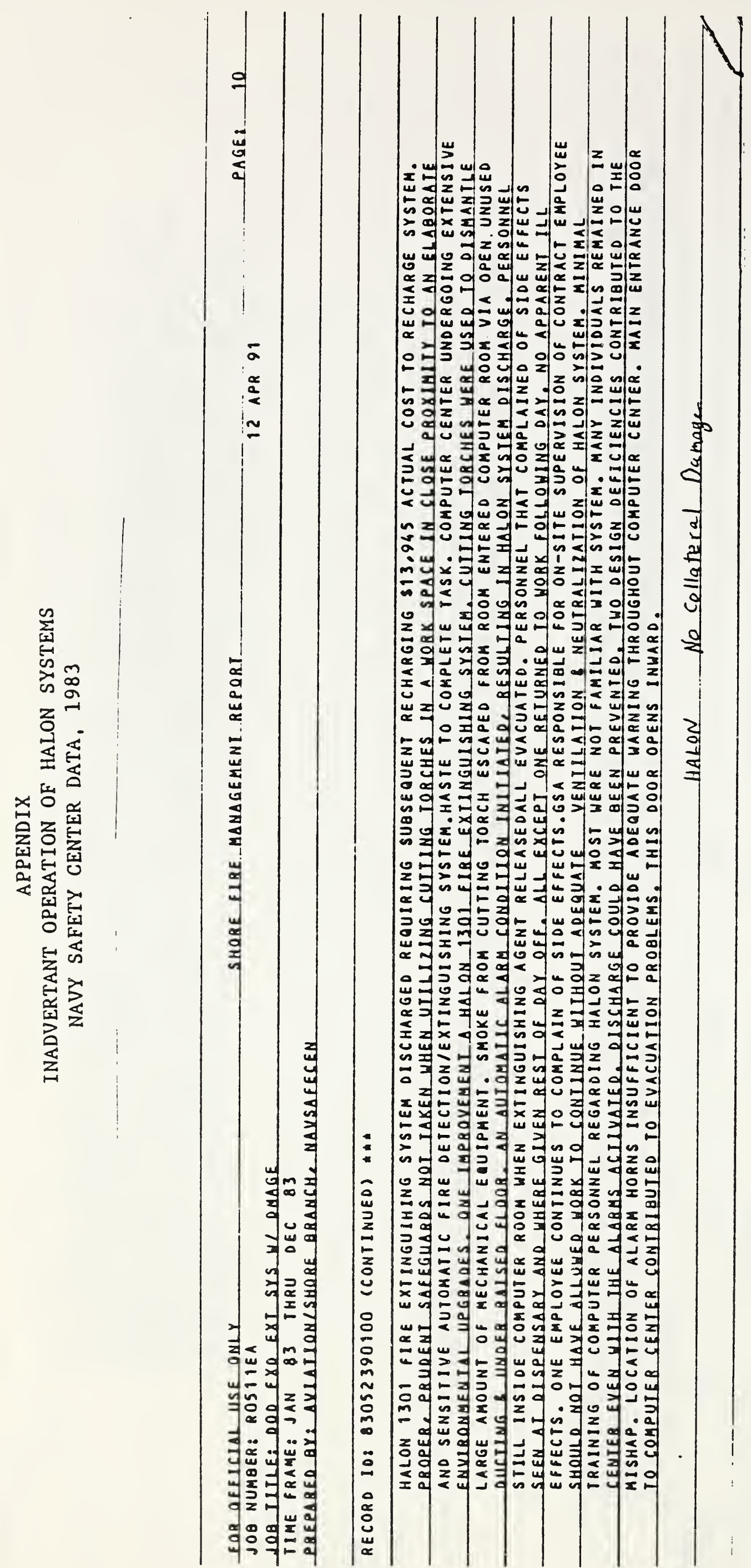




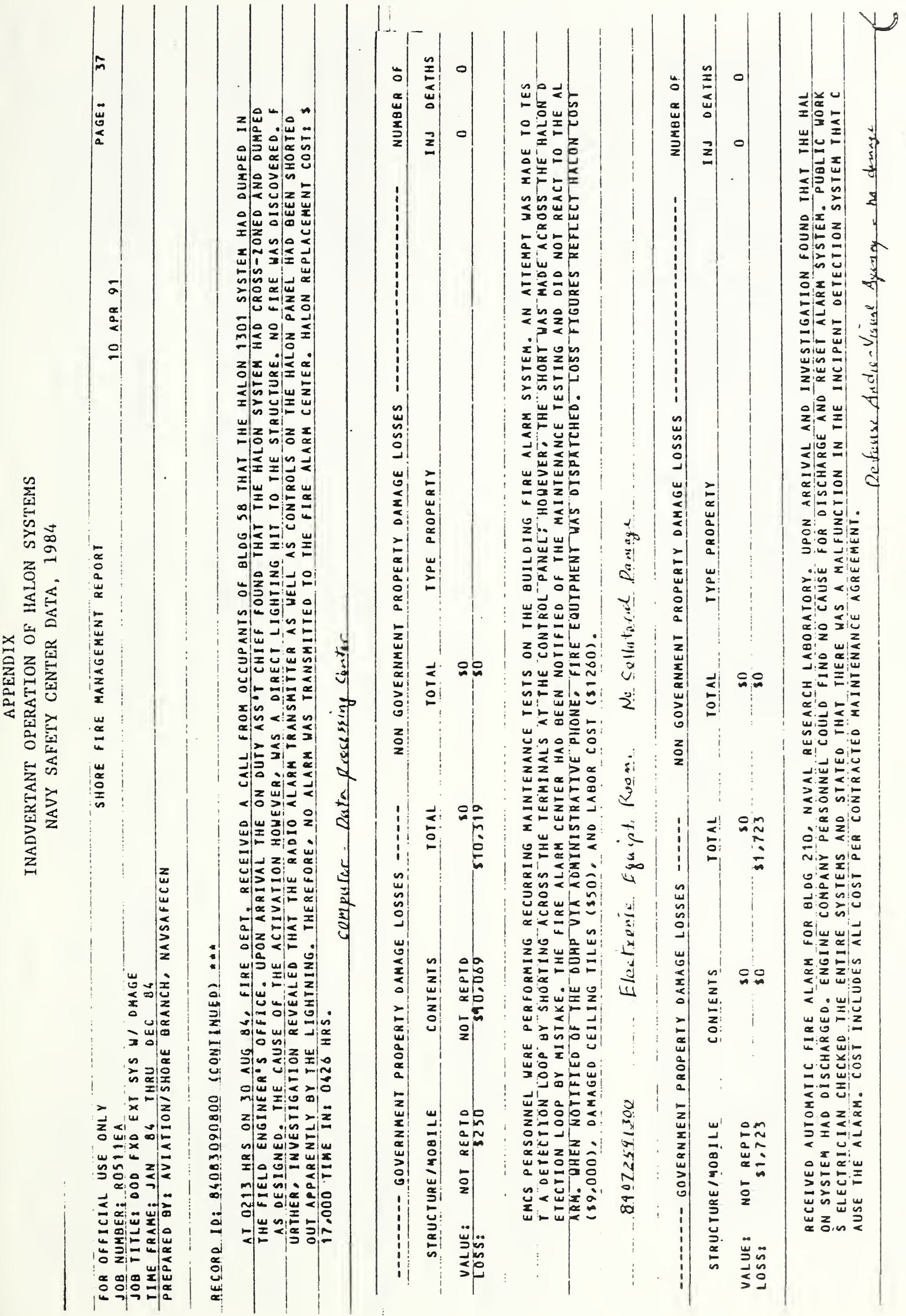




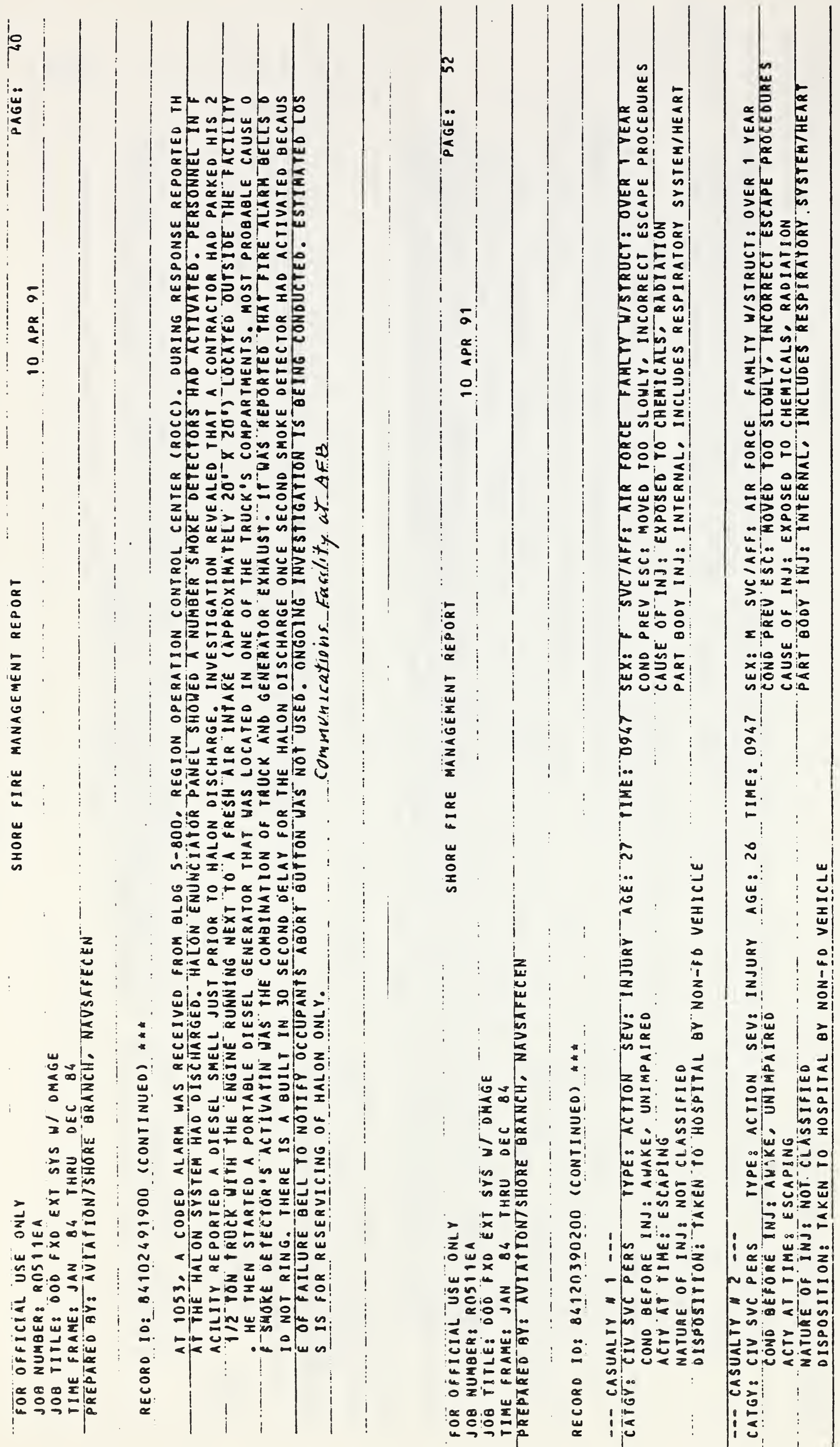

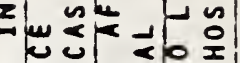

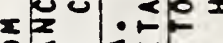
ozzuan 는앙 比空

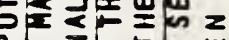

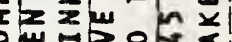
任

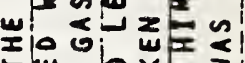
w zÉ의 <这 no

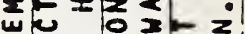

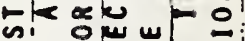
$\rightarrow$ - un I 叫, n n

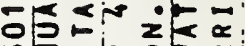

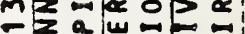
的任长

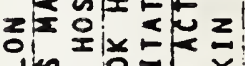
ses

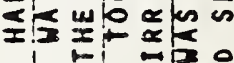

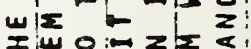

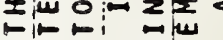

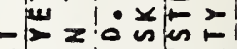

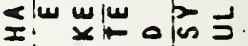

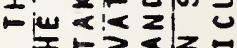

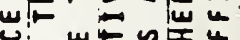

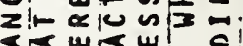

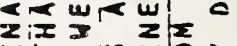
w n n

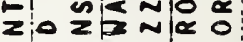
年

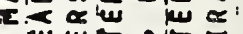

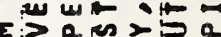

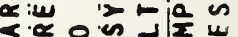
: x. ذ10 <:

I:

둘

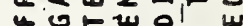

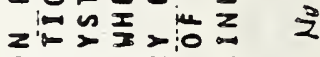

음의

니의

$\leq z w_{0} \propto ⿻ 上 丨$

"

둔ㄷㄴ

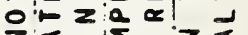

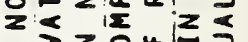

แ정워의

zた́

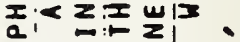

山上た

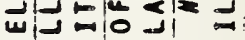

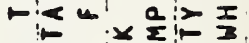

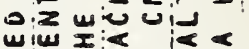

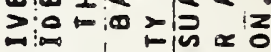
wim

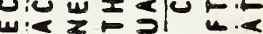

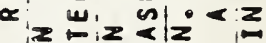
iu s. क्ष ग. 이이 $=12<$

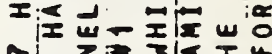
"w2 。ू๊ 눈 


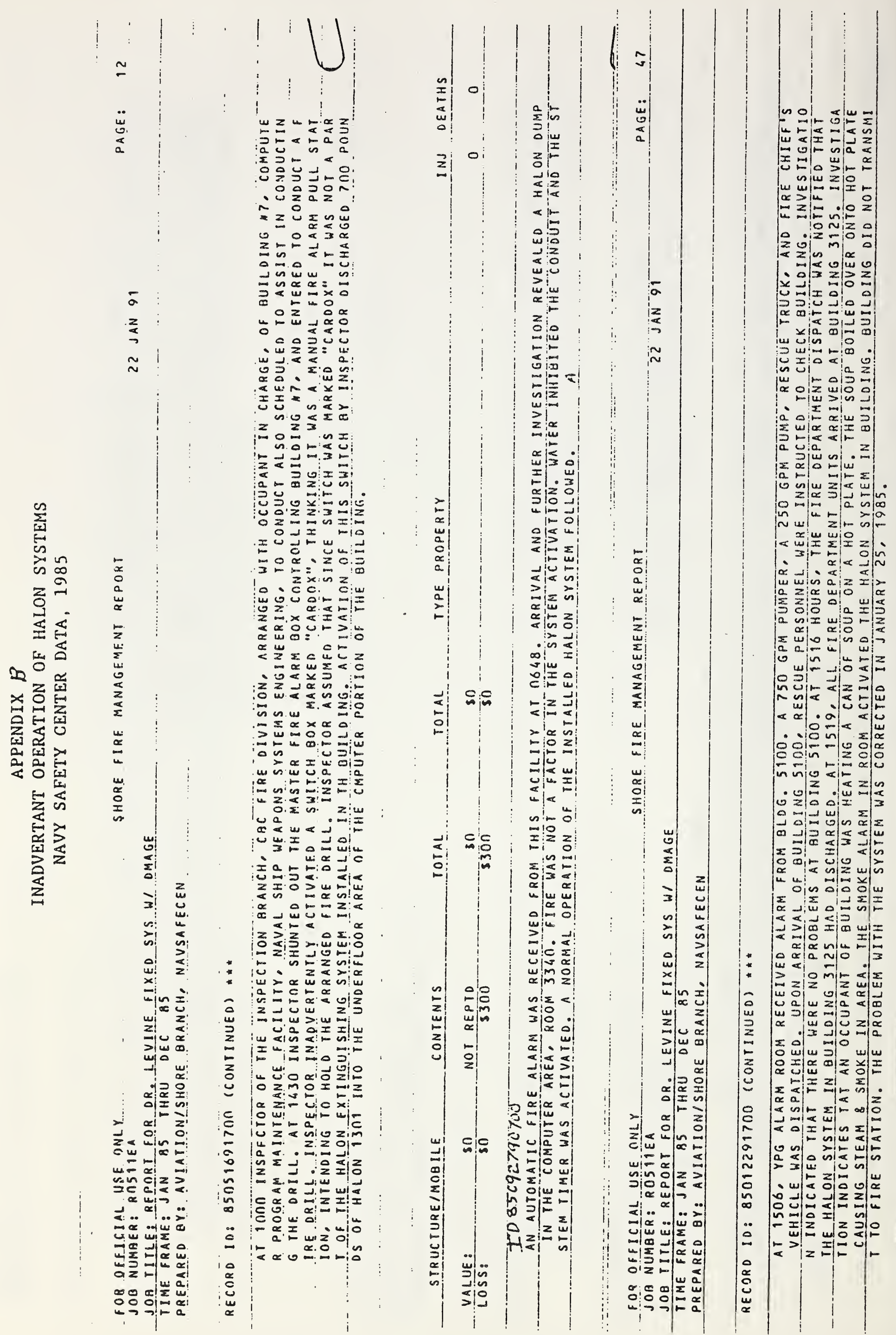




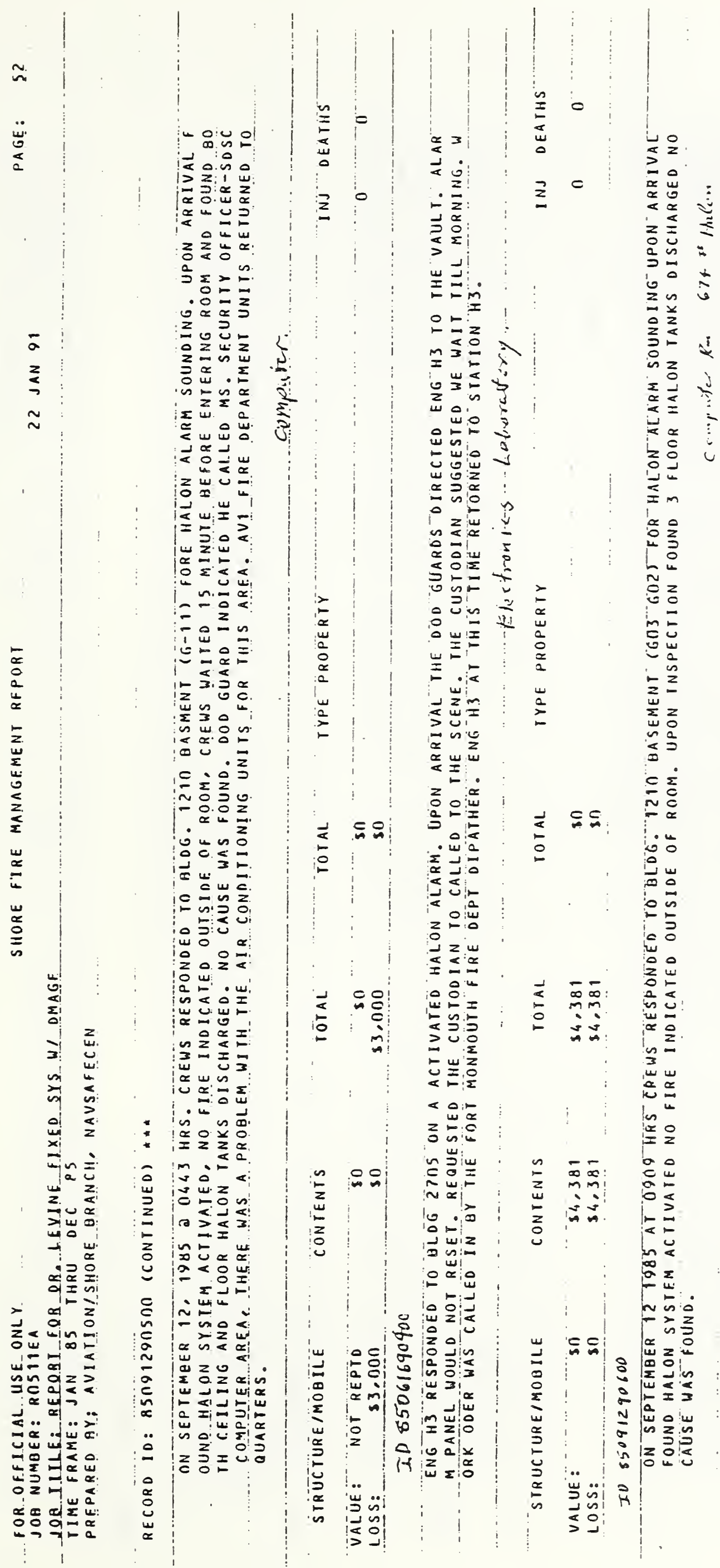

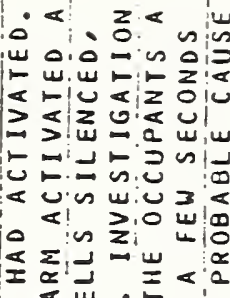

I必定

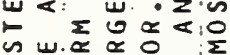

$>\propto ⿻<1$

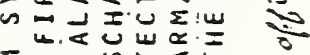

$\Sigma$

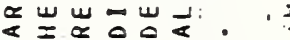

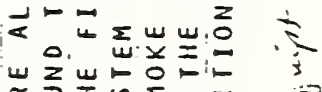

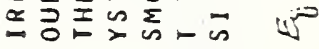

un u $=w_{0} 0$

w.

-

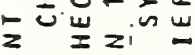

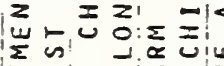

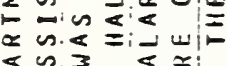

난

○世山心

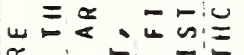

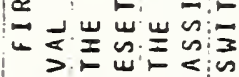

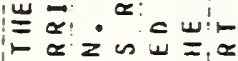

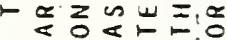

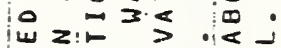

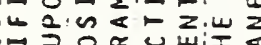

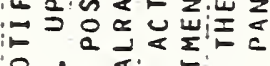

之ं.

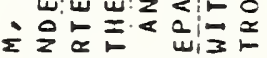

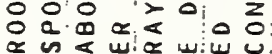

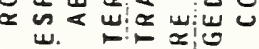

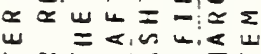

5 w

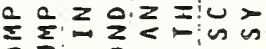

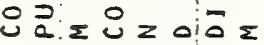

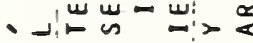

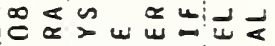

$\infty \geq$ us $>$ a

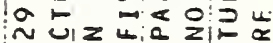

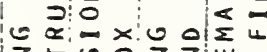

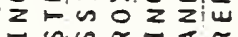

$0<$

lá,

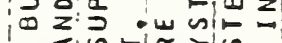

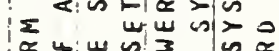

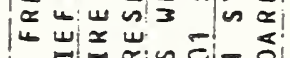

江

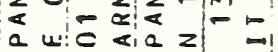

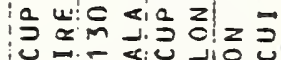

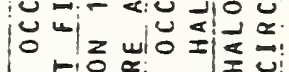

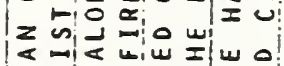

ic ni wis

a

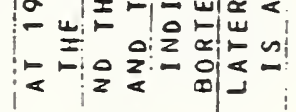




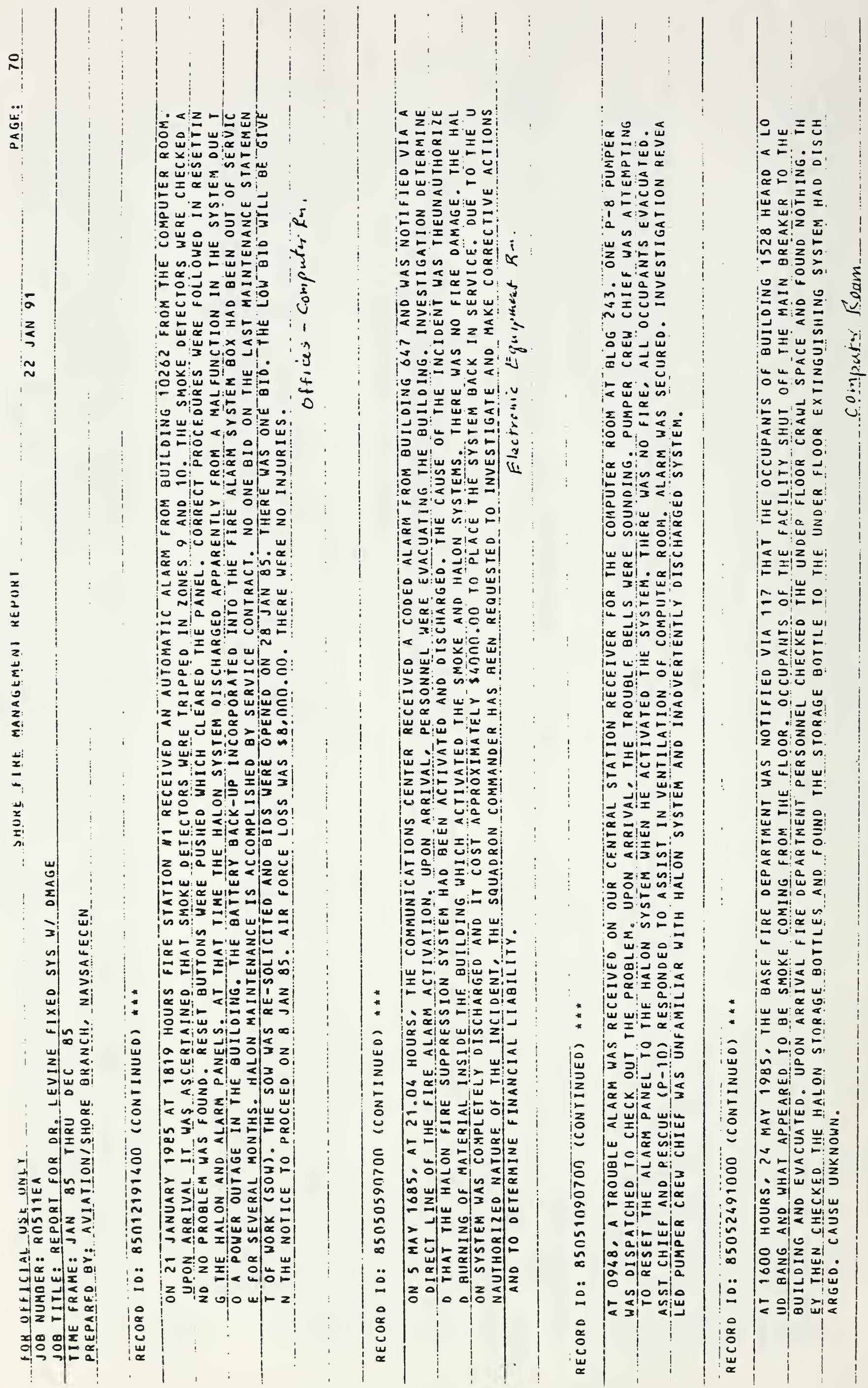


a

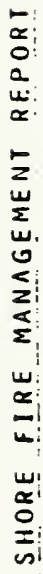

|ٕ।

3

3

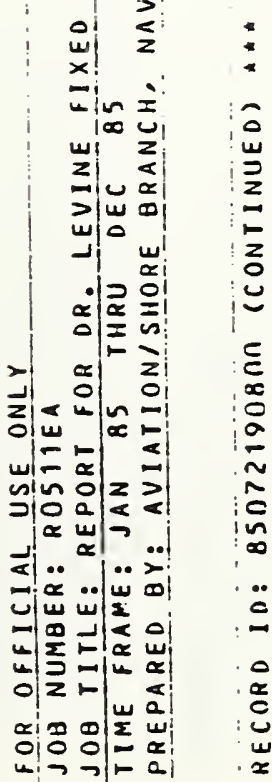

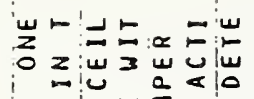

ํำ

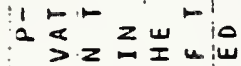

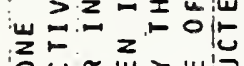

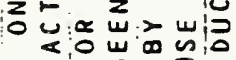

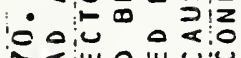

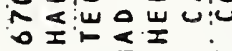

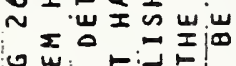

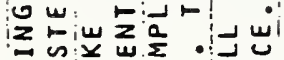

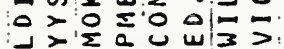

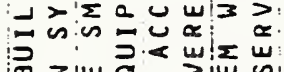

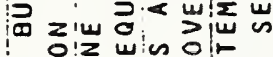

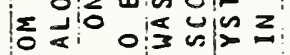

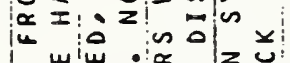

嵌嵌

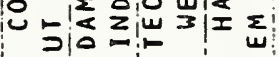

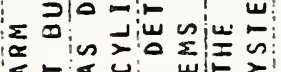

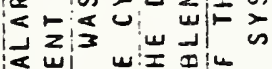

< w z

级出

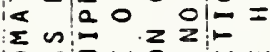

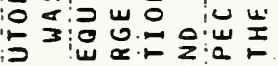

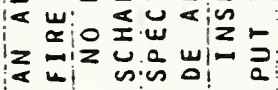

운은

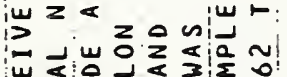

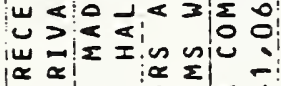

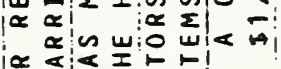

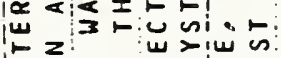

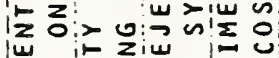

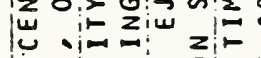

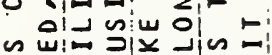

zo ou

인

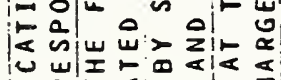

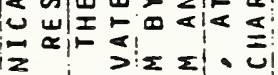

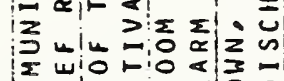

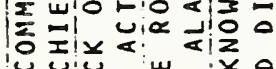

u

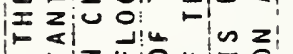

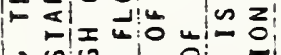

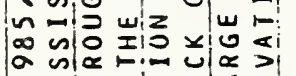

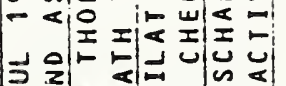

$\rightarrow<<$ 닌

-

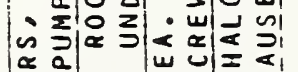

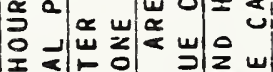

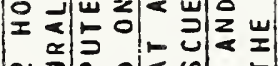

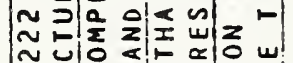

N 깅

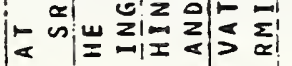

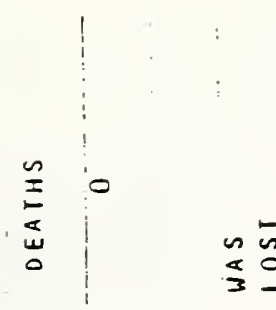

这

ज立

的资:

-

is $0: 1$ 's

is

造衣

的次

世

연

$\leq \geq$

क ज

w

$=5$

\begin{tabular}{l|}
1 \\
2 \\
$\alpha$ \\
$a$ \\
0 \\
0 \\
$a$ \\
$a$ \\
2 \\
2 \\
2
\end{tabular}
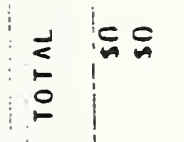

$\underset{\square}{\square}$
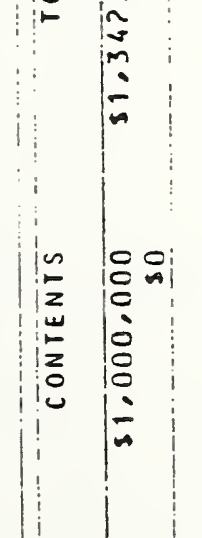

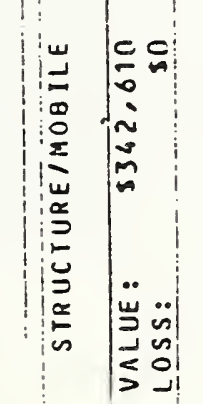

. 210

定这

an

कि

<!

$\therefore$ 嵌

잉중

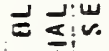

₹

sis $\alpha$

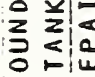

is

(

要 战

a

ज̃u

o

殸

w

岸 a

我

: जी:

u ㄱㅇㅇㅇㅇ

新文

<桨业

玨

过

한의응

구돈인

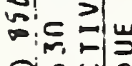

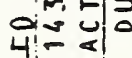

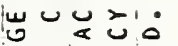

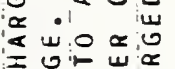

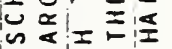

I00'

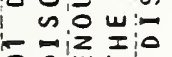

z 2 I U w

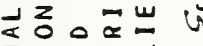

I n⿴囗十丁

<我兰。:

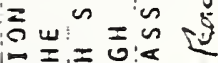

四它司。

巡凌记

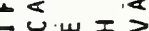

- 00

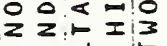

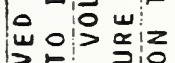

-

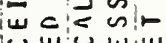

山一⿻上丨

z记选

记爻出

出已:

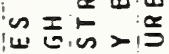

늠의

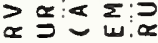

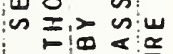

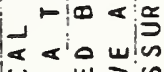

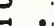

z

势此

政

a $=\sin ^{\circ} a^{\circ}$

o.

wa

4:

的文|山

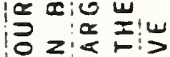

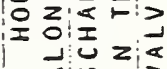

$*$

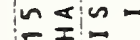

in $=0$

ô

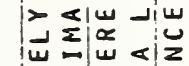

$\left.\right|_{x \mid 3}<$

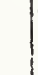

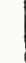

제의.

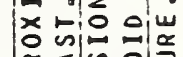

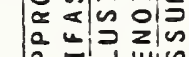

经

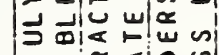

일

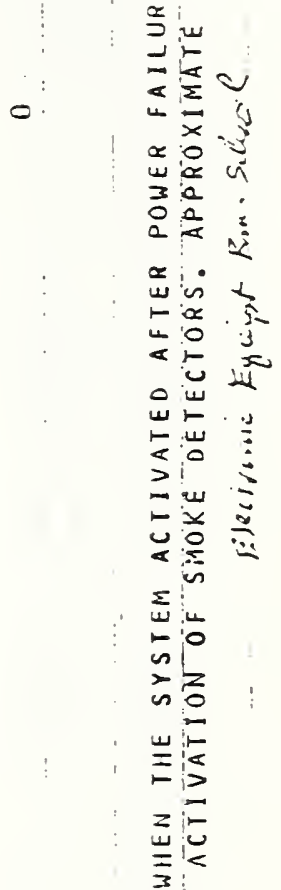

踏

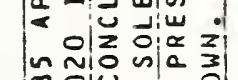

a

万o:

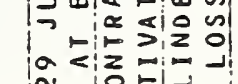


: 2. 3 u 은

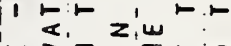

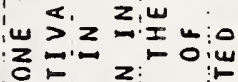
. ico.

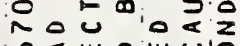
NI ט $=0$ = 结

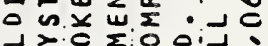
च क्ष 可: $\alpha x_{1}$. os nis $x$ wo $0^{2}$ ois wIiu io

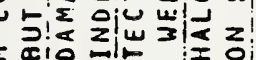
$x$ ci:

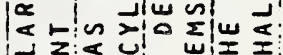

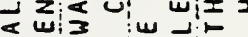
< wi will $\vec{\sigma}_{i}$

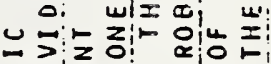

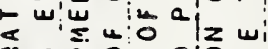
nac c:0

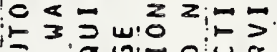

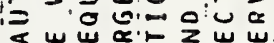
a : a.u<'a n

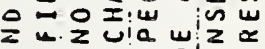

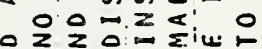
u㧒

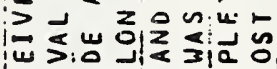

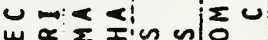

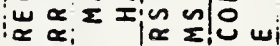
ia $<$ wí wi

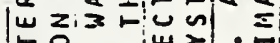

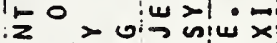
U :

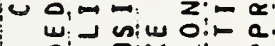

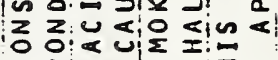
站

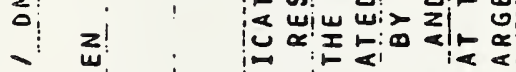
I: u! ! $\quad$ u

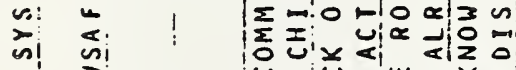
n!

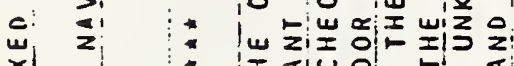
" : $\infty$

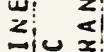
$>$ का \lrcorner$_{i} \stackrel{\text { व }}{\circ}$

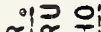
a: >

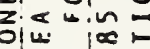
$=\infty$ unie di $|\alpha \omega| \rightarrow$ ¿ा . थ . . a 빙 닐

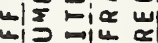
O: 2 -

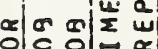

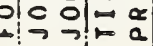

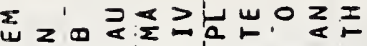
in is win w \&

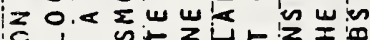

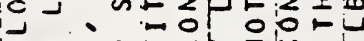

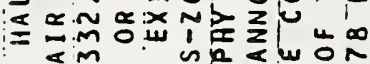

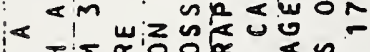
i

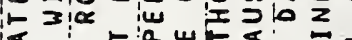

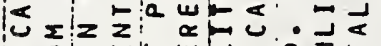

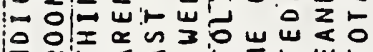

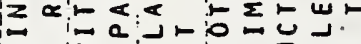

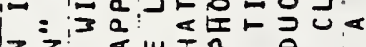

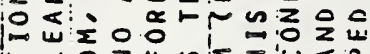
t ड:

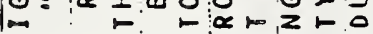

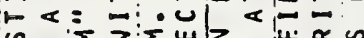
س س س س 及一⿻上丨 - N -mix $x$.

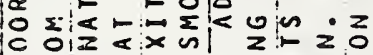
a $0: 0=w \quad z=2$ :

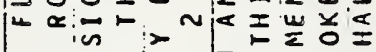

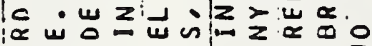

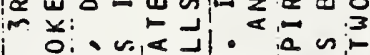
识完活

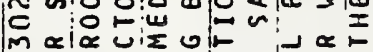

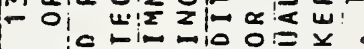
0 w它

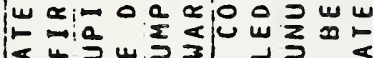

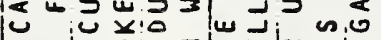

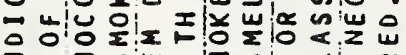

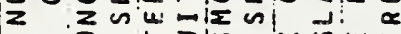

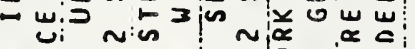

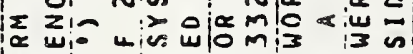

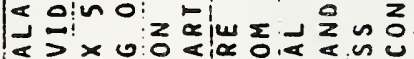

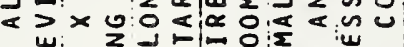
coinz:I n

- I」 1 i

< 在 w

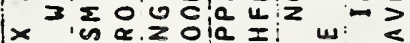

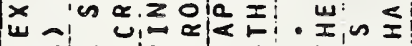

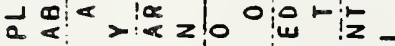

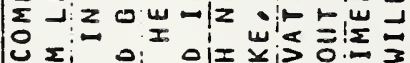

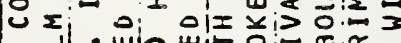

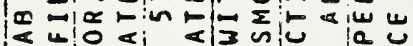

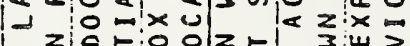
$z=10$

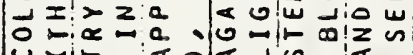
z $\left.\mid \begin{array}{l}2 \\ 2\end{array}\right)$

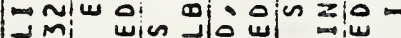

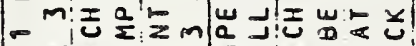

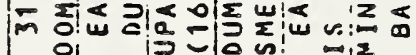
$x$ 作过

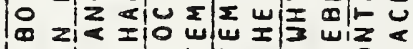

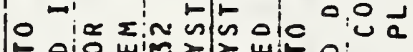

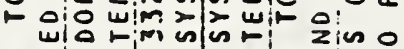

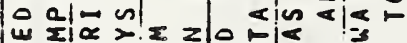

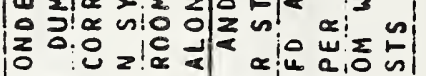

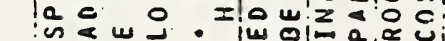

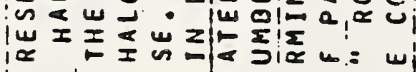

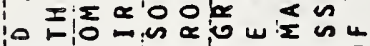

1

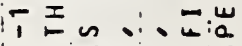
a

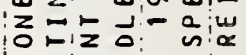

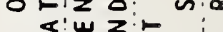

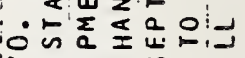

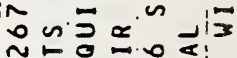
$\sim$ ט $z=\sim z ! 0$ z온이릉 넌

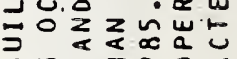

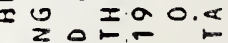

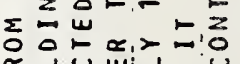
๙ , 岩是

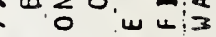
远 $0 \alpha$ os $z \alpha$

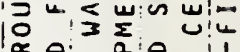
س

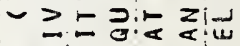
w ○山し०口w。 $u x<z=0$ $\sum$ w $2 \sum$ sit

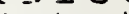

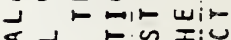

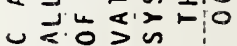

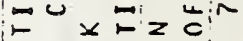

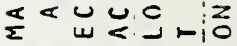
on工 $\leq$ n!

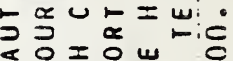

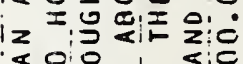
i《o o o

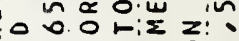

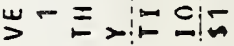

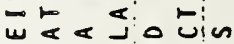
u waw

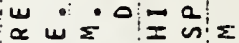
일이 wo

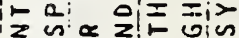

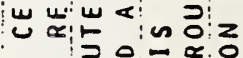

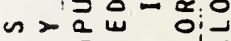

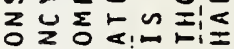
E U U

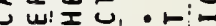

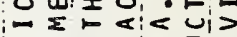
z

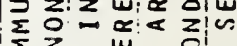

(1) $0<\omega$ is

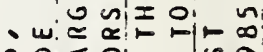

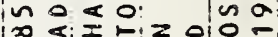
o 존 는 인 บ는

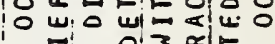
0 I 0 z 1. ज位

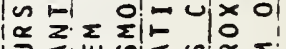
ग w I nim ol a sia

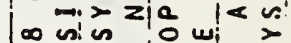

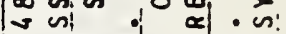
- 4 증 에른 

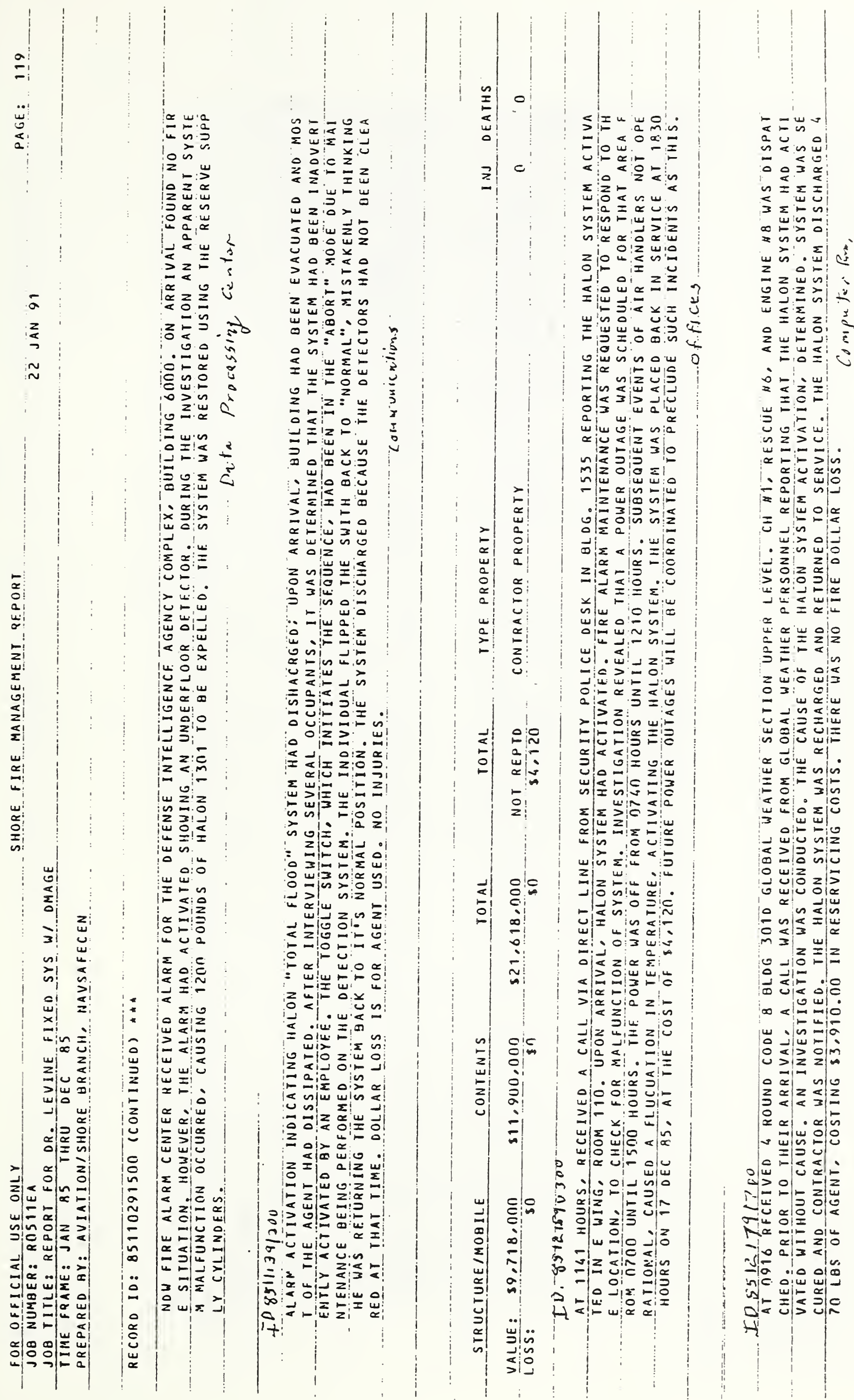


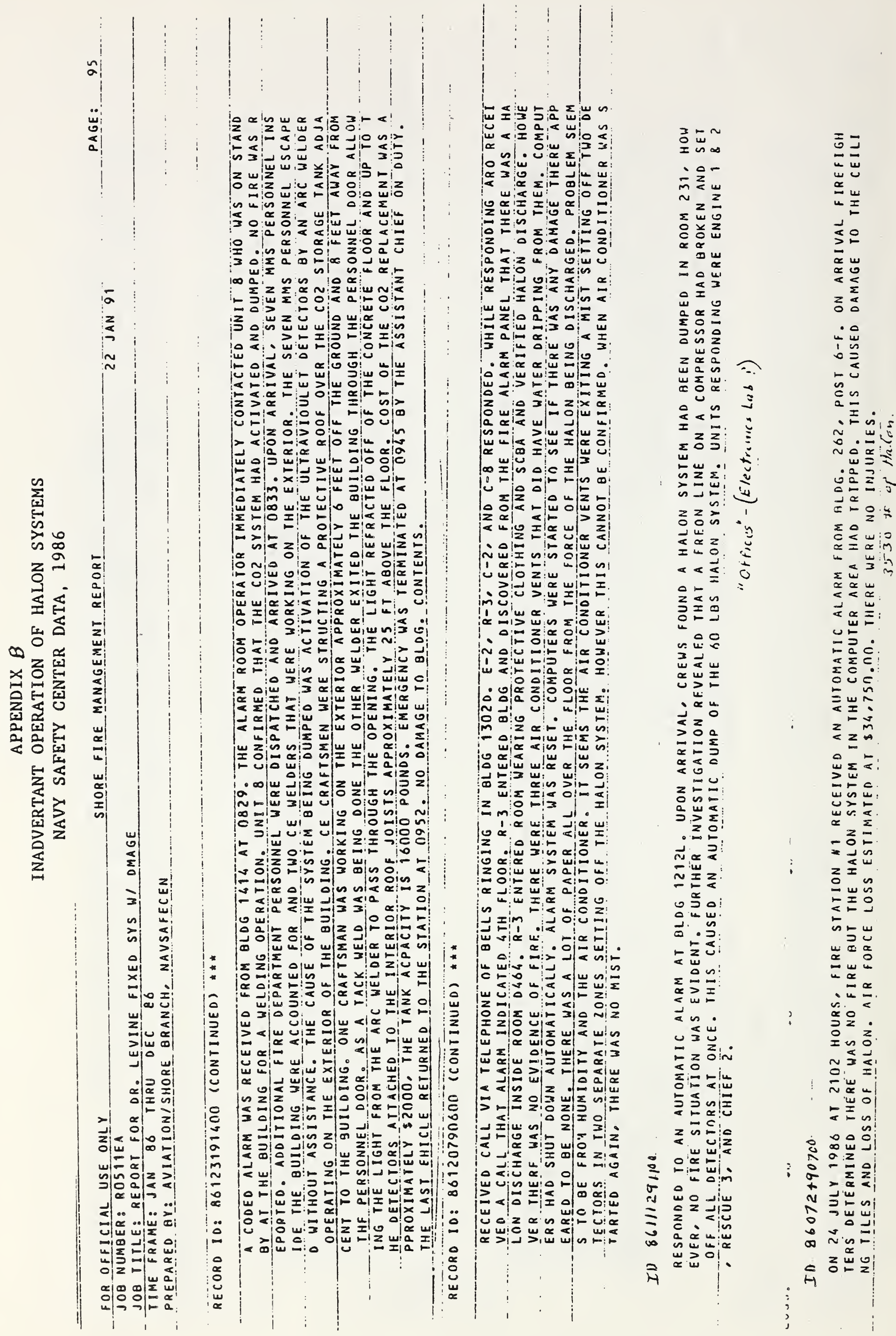


a

3

$\tilde{\sim}$

운

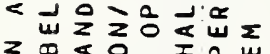
$20<0$ 진

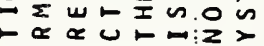
こ电出口国方

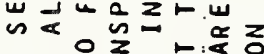

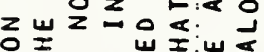

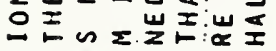
に、艾㟔コ。岂

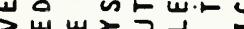
山必的长灰.

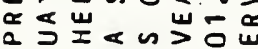

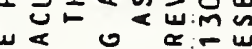
w) 㟧

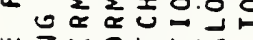

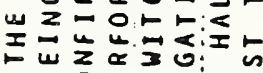

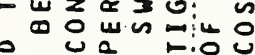
in w a n

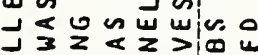

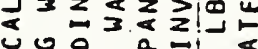

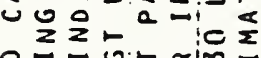
응 $\overrightarrow{4}$ is:

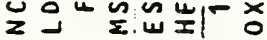

w

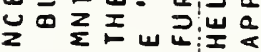

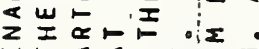

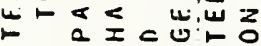

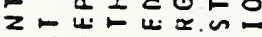

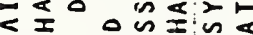

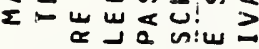

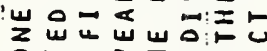
이는

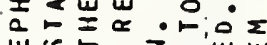
س ーシのにコ的文

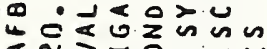

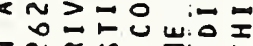

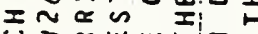

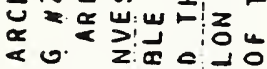
×

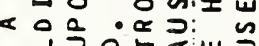

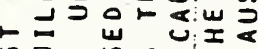

宁

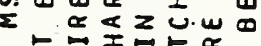

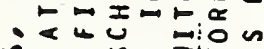

我

a

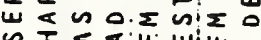

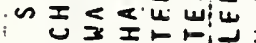

$\checkmark$ U

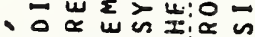

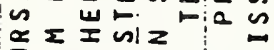

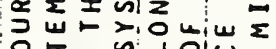

을 的的일

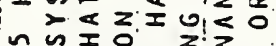

行的壬山经的

a

$\therefore$ 궁음

o, 的

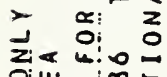

幽

win 이리

ग0 a

व

-

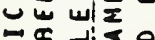

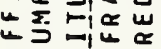

○े च

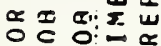

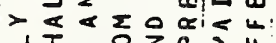

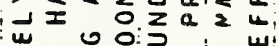

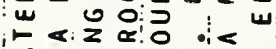

造

: 0.2 w 0 गो $\alpha$

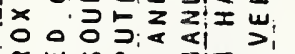

呚出 n

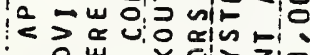

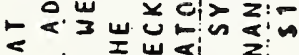

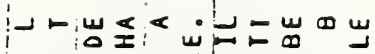

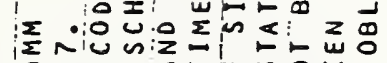

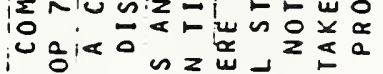

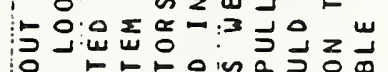

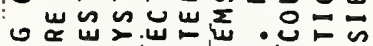

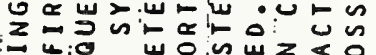

ㄴ:0

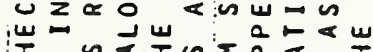

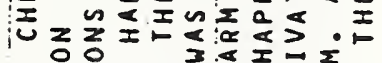

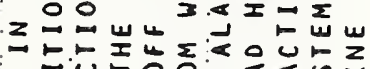

-

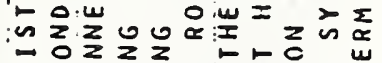

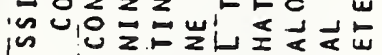

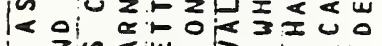

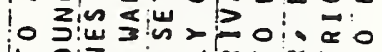

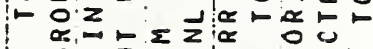

๔ 心

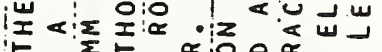

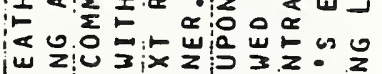

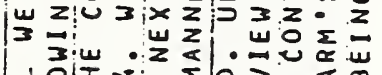

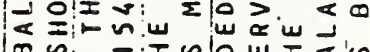

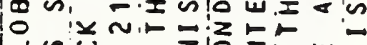

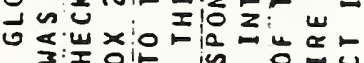

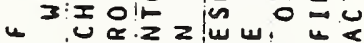

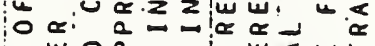

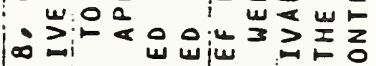

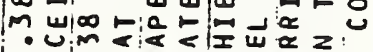

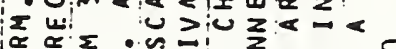

1 0 인

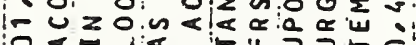

M

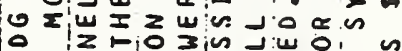

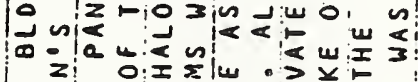

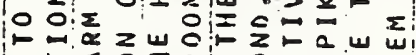

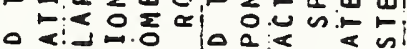

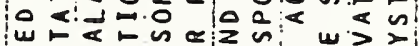

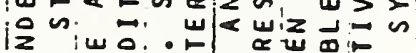

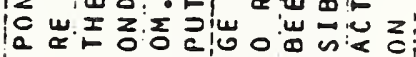
出 ๙

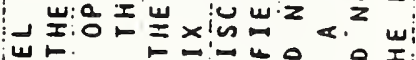

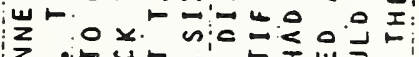

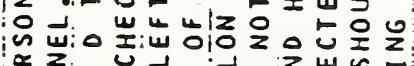

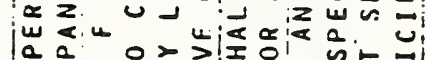

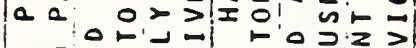

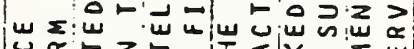

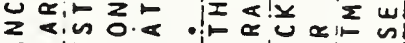

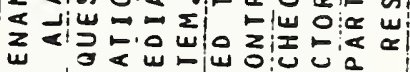

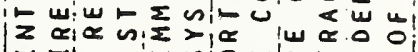

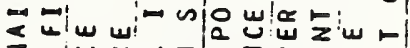

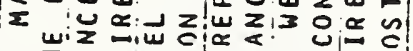
w

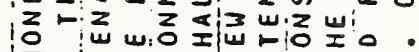

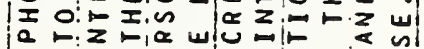

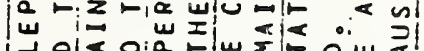

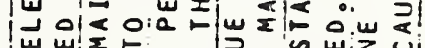

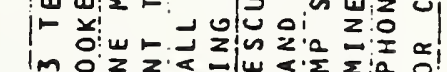

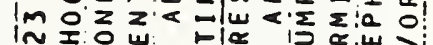

I

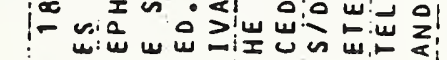

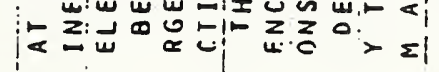

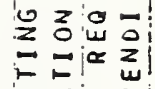

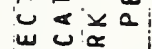

w

우욜

a o

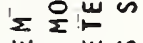

崖出

汶的

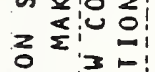

本我全它

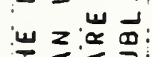

岂飞得思

ज伿

造品嵒

行它分出

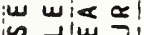

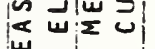

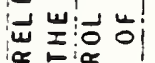

z w:

四家

$=\overline{3}$

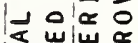

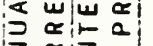

这赑

年的立

₹z心잉

은

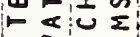

约约

D50 w

एᄂ $<12$ 的

|

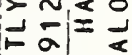

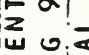

o zí:

ज安远

네의

z舟出

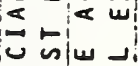

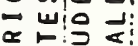

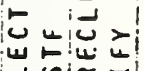

山ै:

? z

< 다

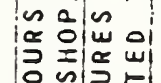

온

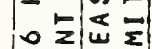

का 닐

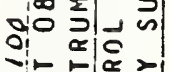

चए जाए

는 2 ज

vo $=5$

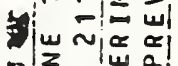

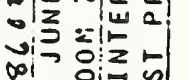

व

4

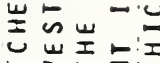

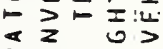

礼元.

무는

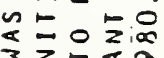

z

z.ํㅝㄴ

世幽出

$\sum w 0 . n$

的上就

品的卡立

य气

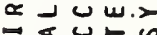

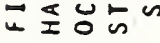

这岂的览

$\propto$

బ

ט

근

に三た㟧

인

틴

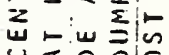

ఏ。

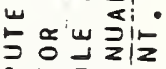

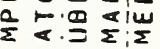

원의

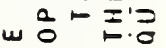

吉以㟧山心

工正上次

ㅇํㄴㅁㅣ

แ 山上に。

w

으의

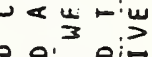

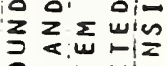

o on wa

네

< 远娄坚

임

$>$ zú

ш खi山

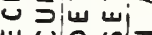

山 ज政

$\propto 0.4 \approx 50$

닐

z $0: 4$ old

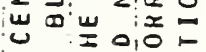

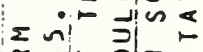

选证我的

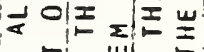

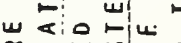

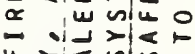

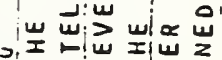

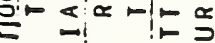

Fiv oiz sin

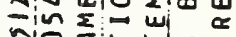

ấ

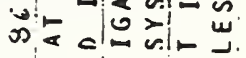

से 

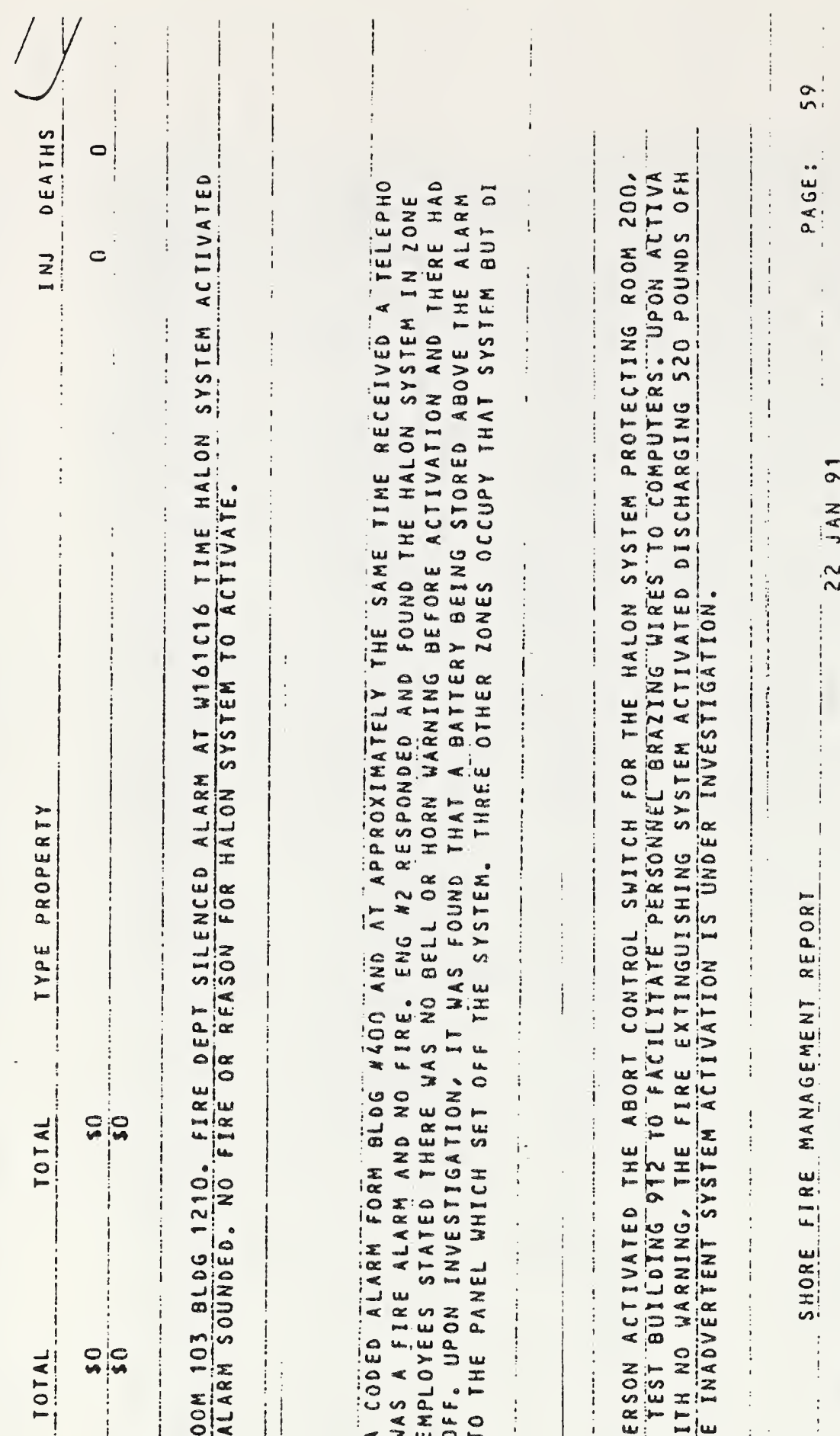

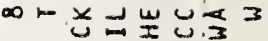

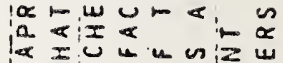

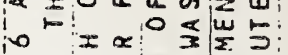

彳 200 :

z

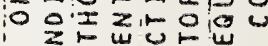

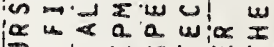

年

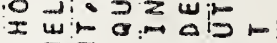

o z $z w$ w

21 i i

C

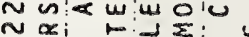

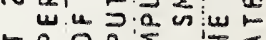

化a.0 a

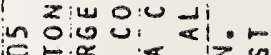

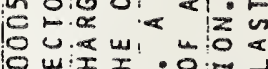

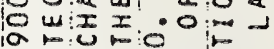

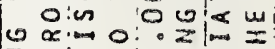

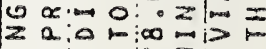

on wim $N z$

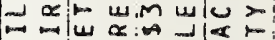

स्य

$\infty>\frac{a}{2}=0$

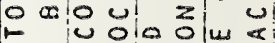

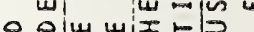

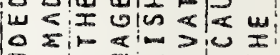

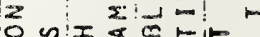

a 10 a

क 3 in on

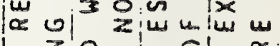

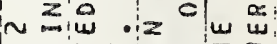

u dir

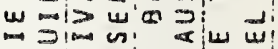

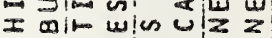

u iv $\alpha$ in

낭

$z$ m 0 w. w

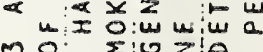

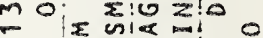

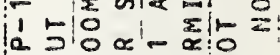

1. 000 w:

o va wiा wlo

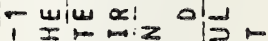

\& : w

is wa

$z-2: 0$

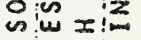

a.

a in 3 沙

$\alpha$ is

$\pi<2<0$

$<$ I I

:

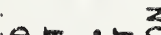

w $u=w$

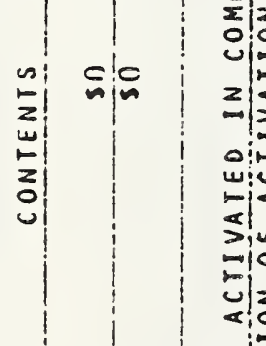

$\alpha<0 \alpha$ แ

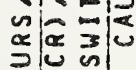

天

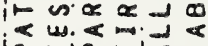

잉 dim

$\omega>a, 4$

i० a 0 出

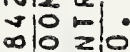

in $\sum_{w}^{2}$ u.

$0, \infty$ o!c

w

<这-

齐!

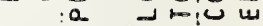

D.

1 ш:

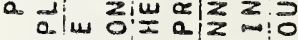

$<\Sigma=-1+<0=$

उi

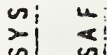

u.

of

$=$



$0<$ :

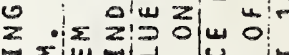

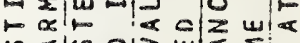

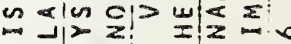

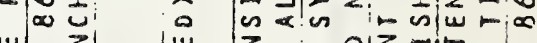

z.

$>$ i $\infty$

(

. 20

일

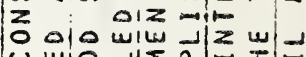

w wो wाए

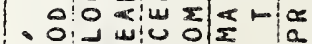

m $u$ u $>\ll<$

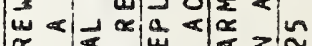

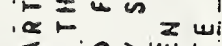

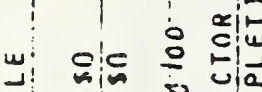

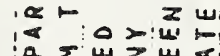

of $0 \mid z$ 济

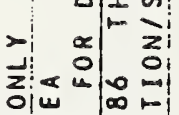

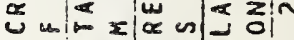

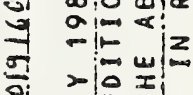

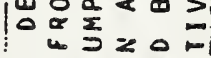

<1: I:

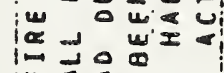

i) इ्र

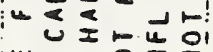

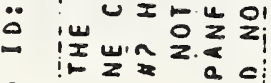

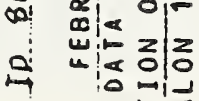

15

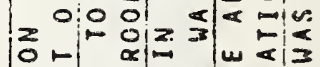

ㄷ․에

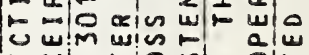

wा -

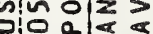

$\propto$ แ⿻

苟

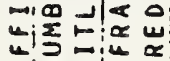

o:z

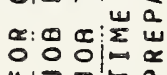

w w

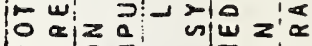

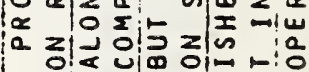

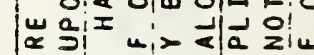

« 


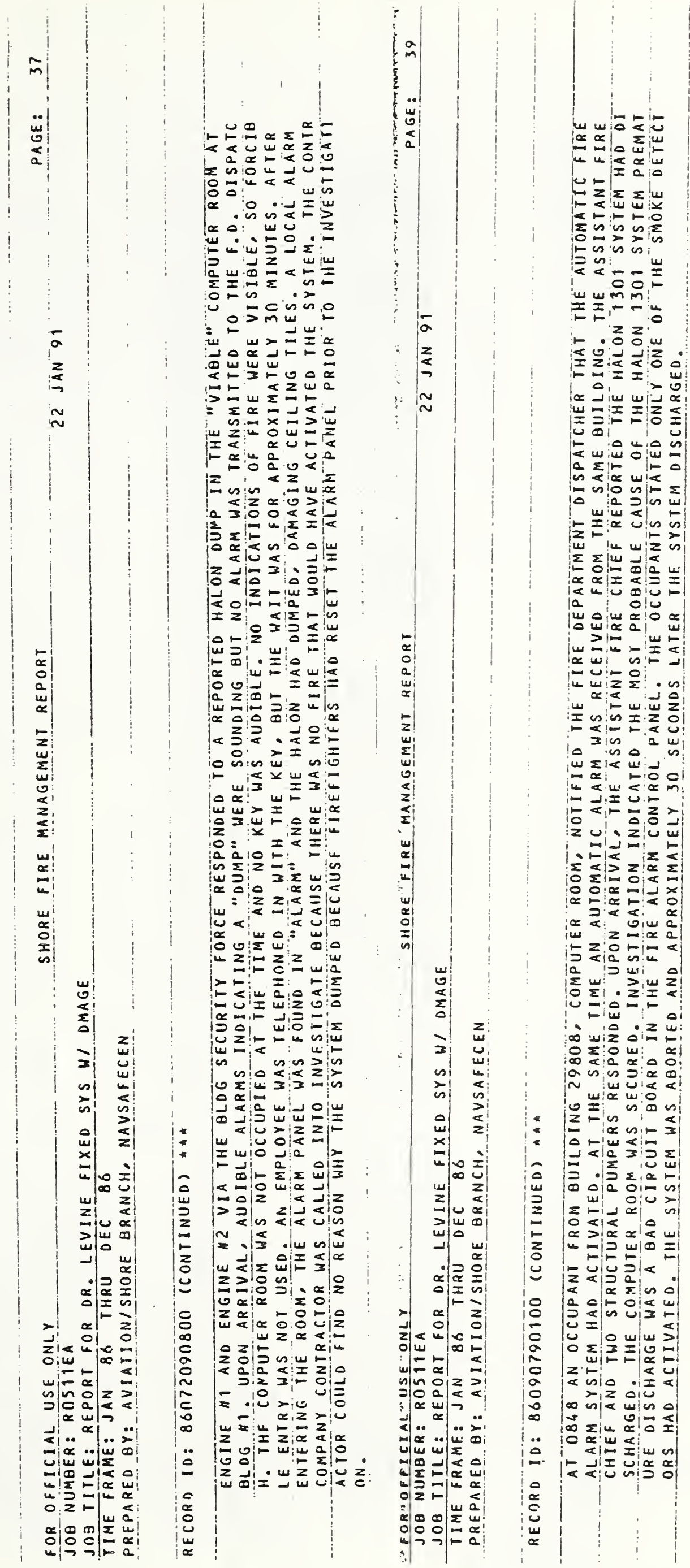




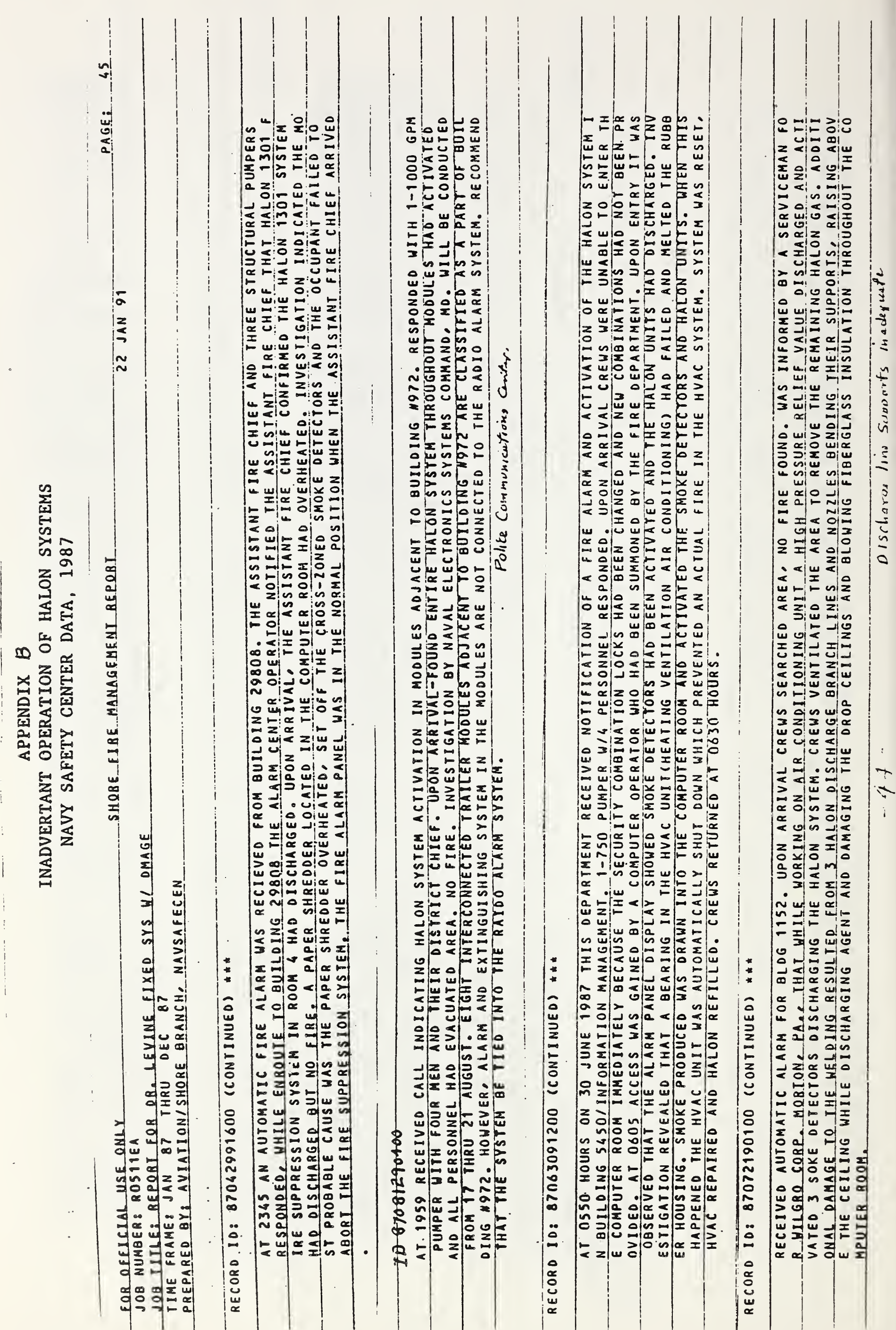


ज.

$\left|\begin{array}{l}\sigma \\ \alpha \\ \alpha \\ \sim \\ \sim\end{array}\right| \quad \mid$

ב⿱⺈

w

x

- 3

3 w

के की

이 를

$\stackrel{x}{4}$

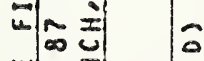

씰 zz

$\rightarrow$ un

$-4$

어룰윛

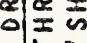

7 잉

이난

$=-\infty$

네의 0

$\Rightarrow 0$ a $<$

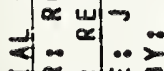

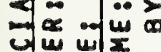

넞

일

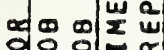

영요의 (a)

웅ㅇㅇㅇ

象造

- 0 a

is $\alpha$ is

吸

乙a

|

<这

ax $x$

एँ.

is

$>\approx$ 皮

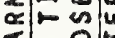

을

$\because 2=x$

$\ddot{0} \ddot{0}$

ज山्य

की $>$

a

$\ddot{x}:$ 出

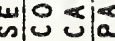

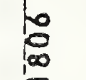

i.

ए人

it

的管

. 이릴

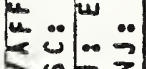

눈

s $\underset{\sim}{w_{0}}$

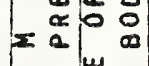

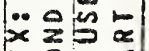

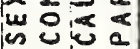

E

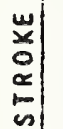

a

in $\quad k \quad m$

is

$<=$

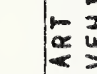

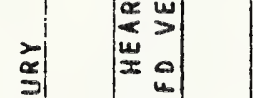

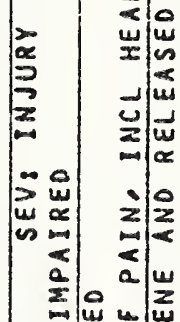

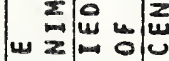

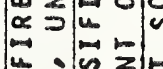

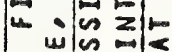

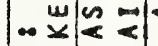

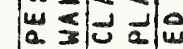

- $x<<0$

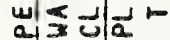

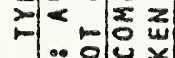

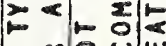

$\vec{z} z$ 닐

$\ddot{z} z^{2}$

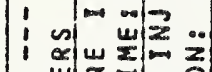

络

- as a

*

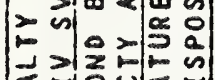

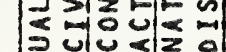

:

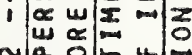

Na.

-

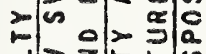

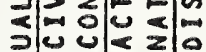

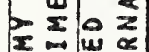

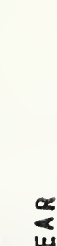

出

$-\infty$

$\propto$

30

.. 중

돌융르

$\propto 0$

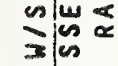

w

$\begin{array}{lll}2 & 1 & 0 \\ 0 & 0 & 0 \\ 0 & 0\end{array}$

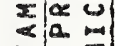

$\propto$

in

2 崖。

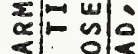

.

.

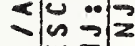

山

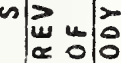

$-200$

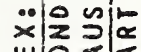

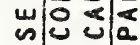

융

:- w

崩

点

皮

n

告

瓷

zे

넌

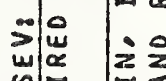

幽

a

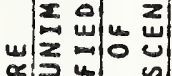

可武比

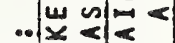

니종

하는

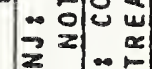

जิ:

:

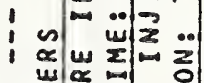

m

- v|ur

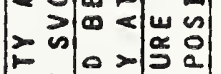

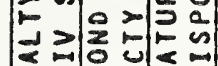

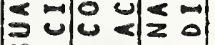

这
10.

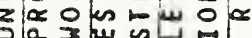

안

.

2:

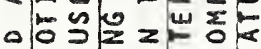

шо저의

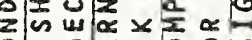

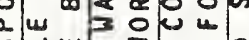

山⿸丆口

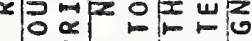

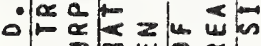

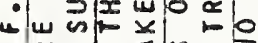

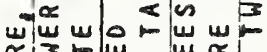

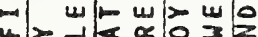

出a

< E

50 w

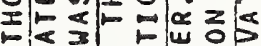

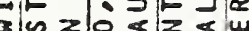

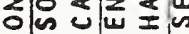

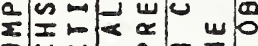

blu

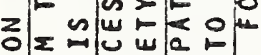

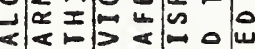

I

$<0^{0}>$ w 0

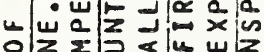

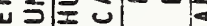

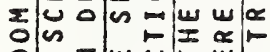

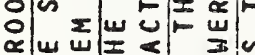

$\therefore$ 王

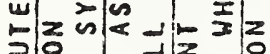

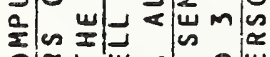

vio a w 20

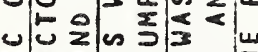

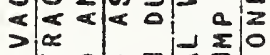

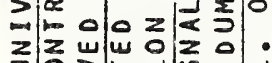

บoर

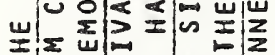

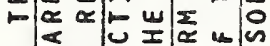

I.

$\propto<\sum \leqslant 0<0$

4

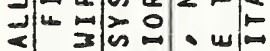

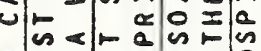

: 0

유 주일

岁位

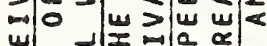

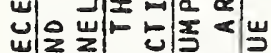

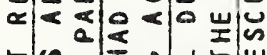

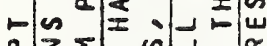

a.

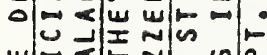

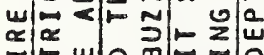

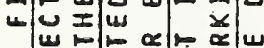

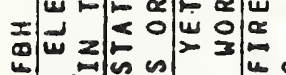

ans as a w

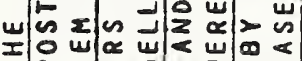

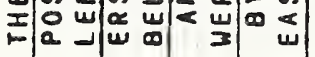

-

ш

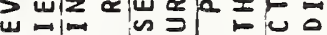

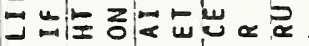

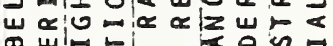

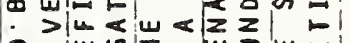

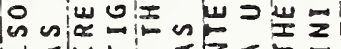

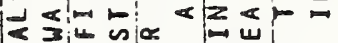

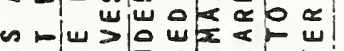

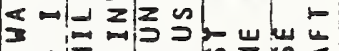

- - vilo il

-

- ㅃ山心

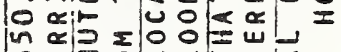

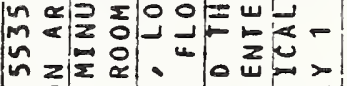

z

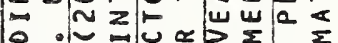

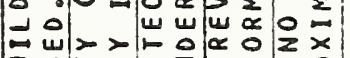

J

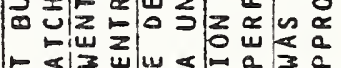

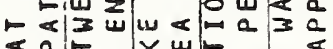

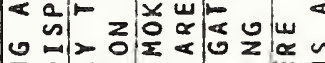

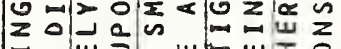

닐 z

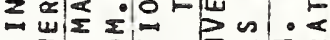

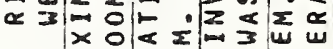

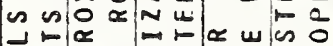

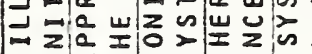

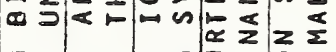

I

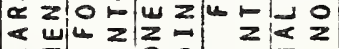

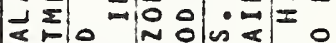

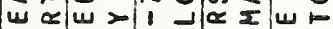

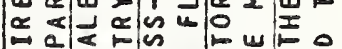

4

u

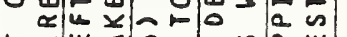

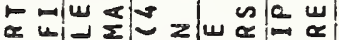

年

w

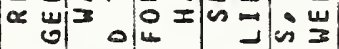

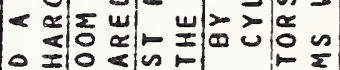

山 U.

س س

u

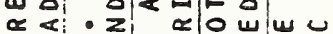

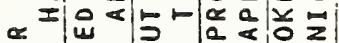

世

年

$\leq \Sigma=0$

a

$\Leftrightarrow \propto 0<0$

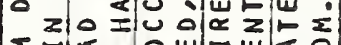

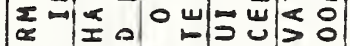

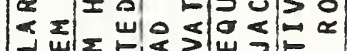

<

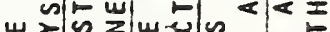

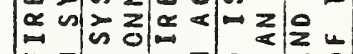

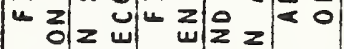

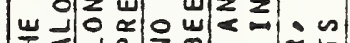

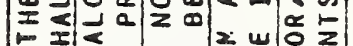

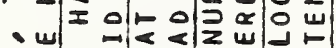

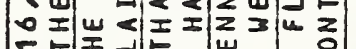

$\therefore$. 


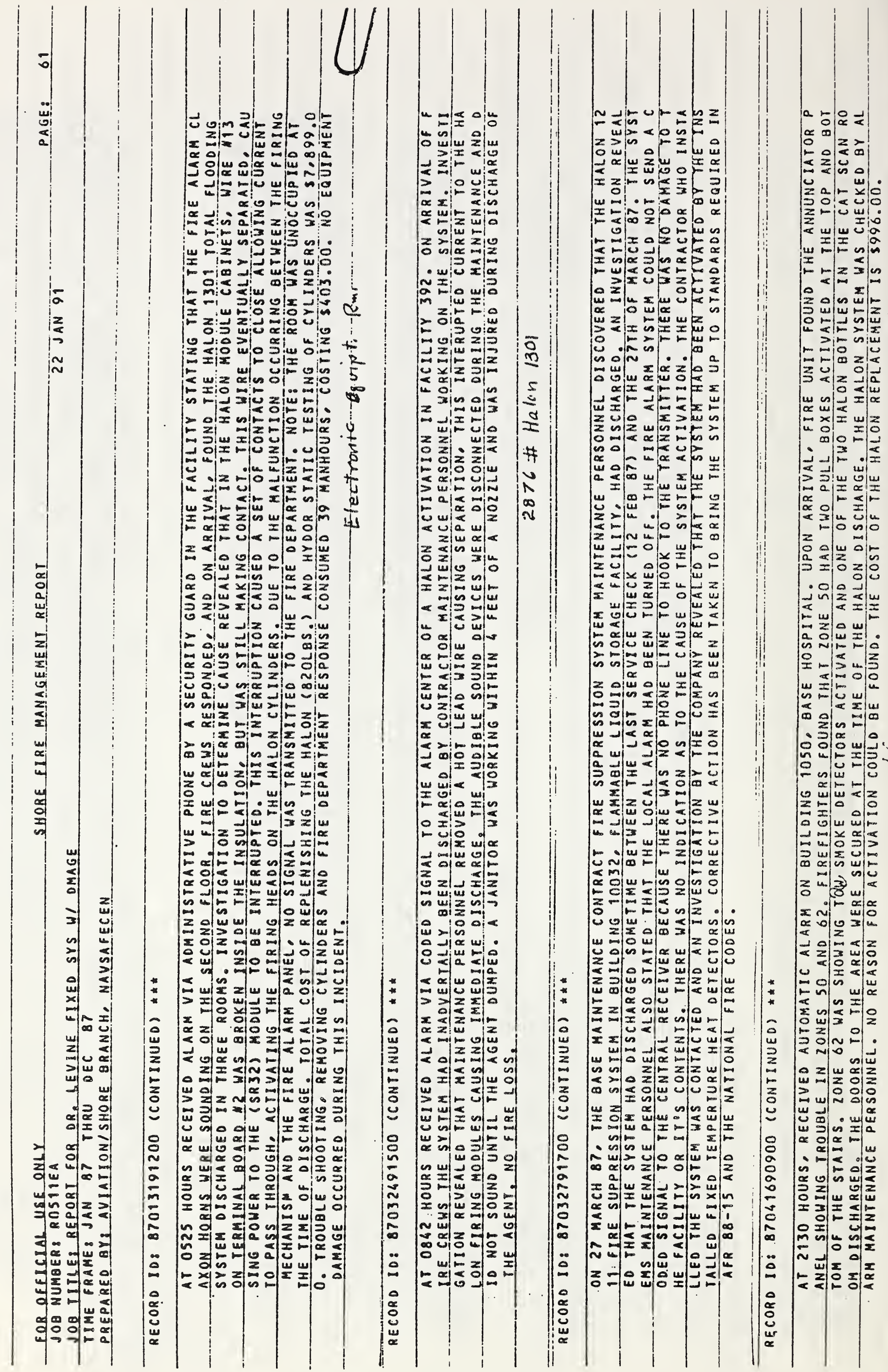




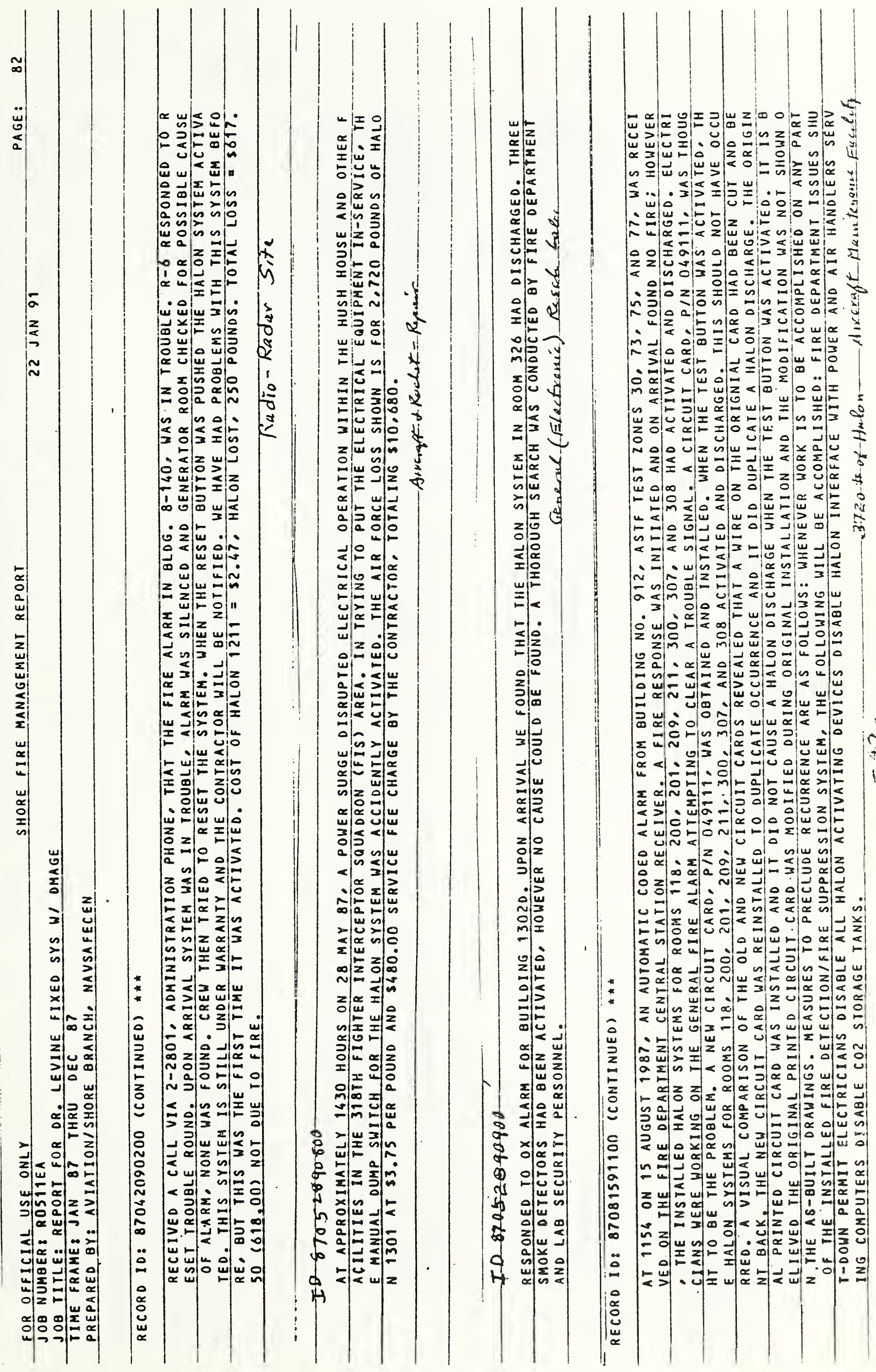




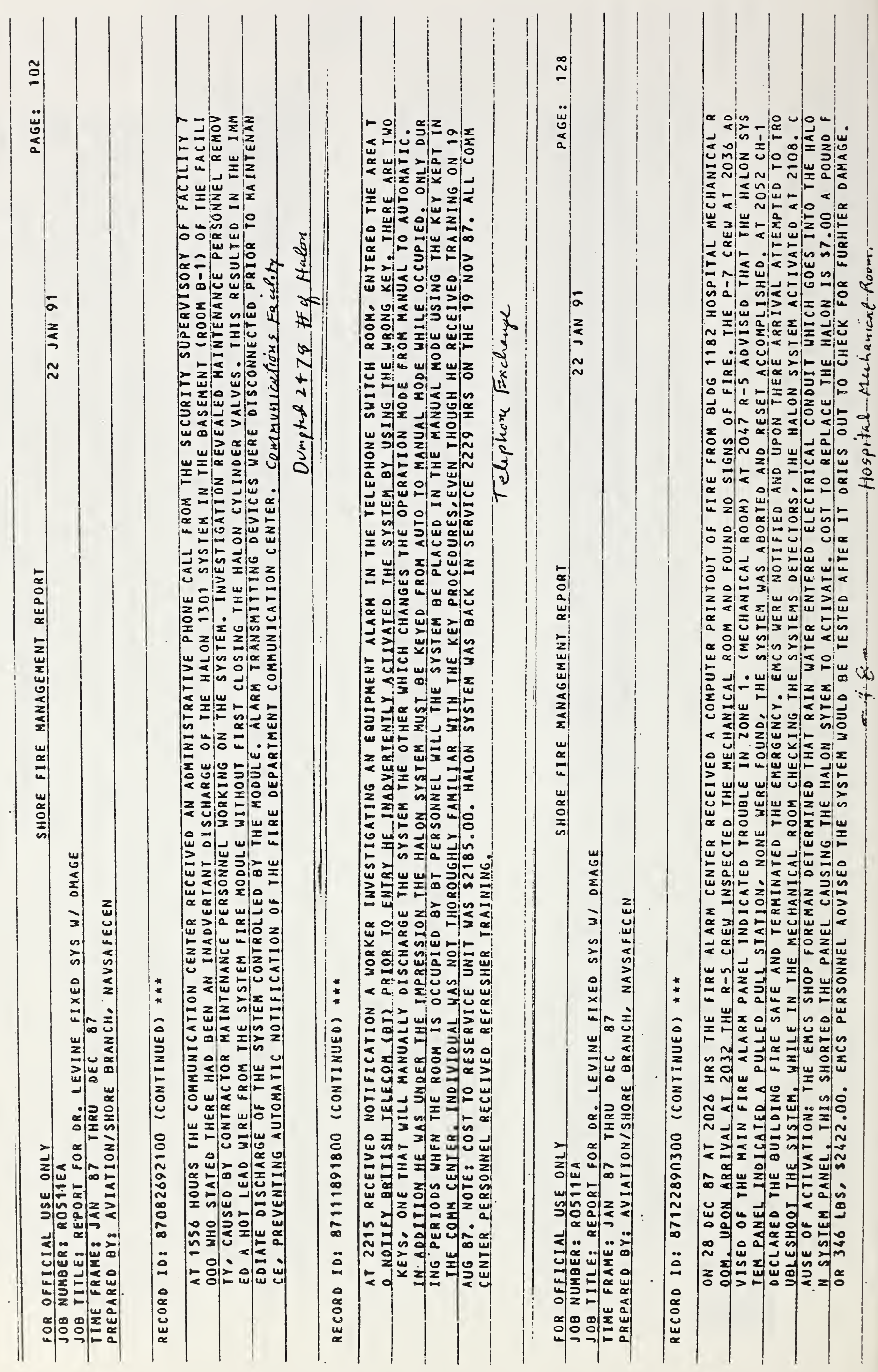




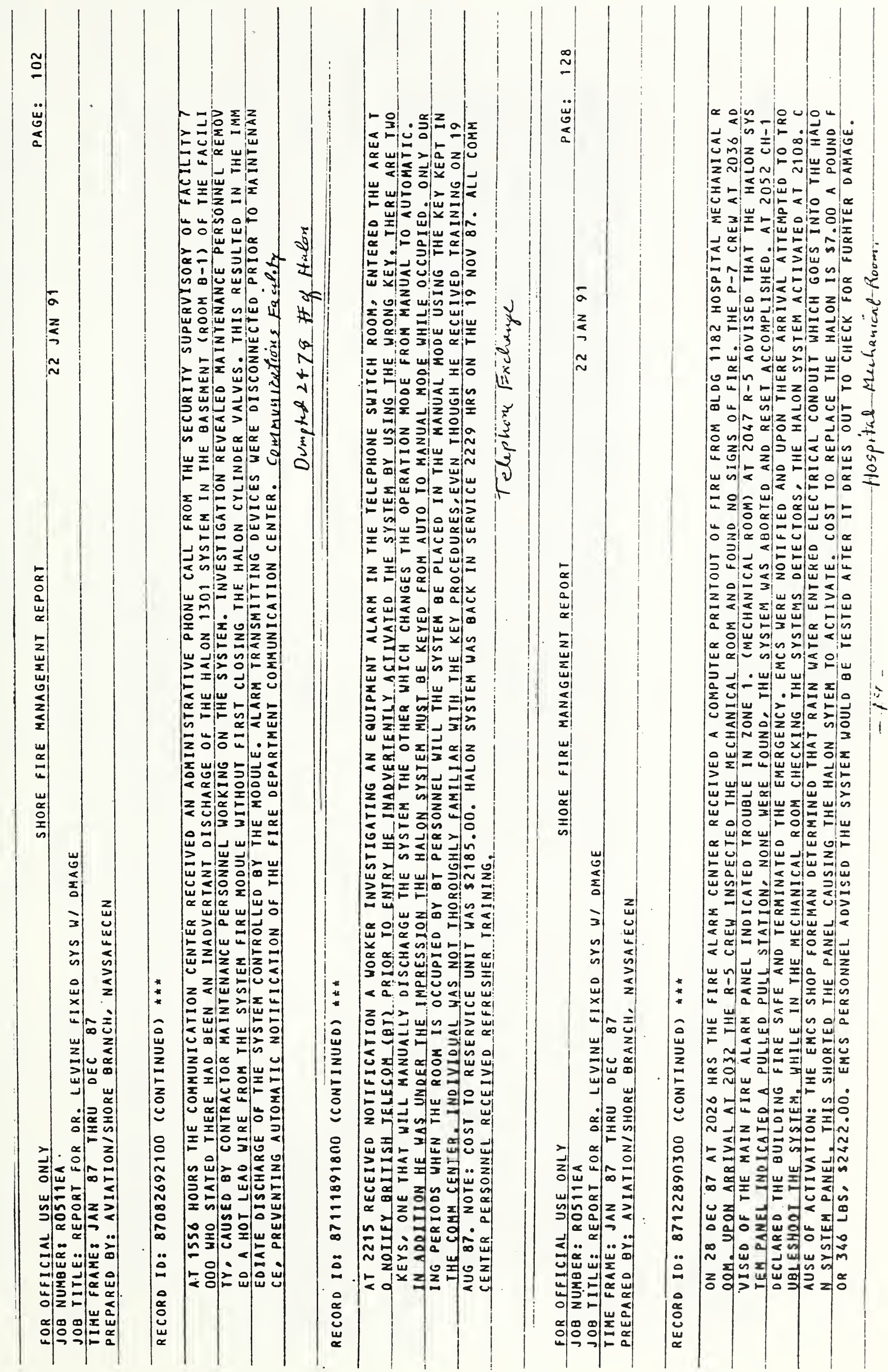




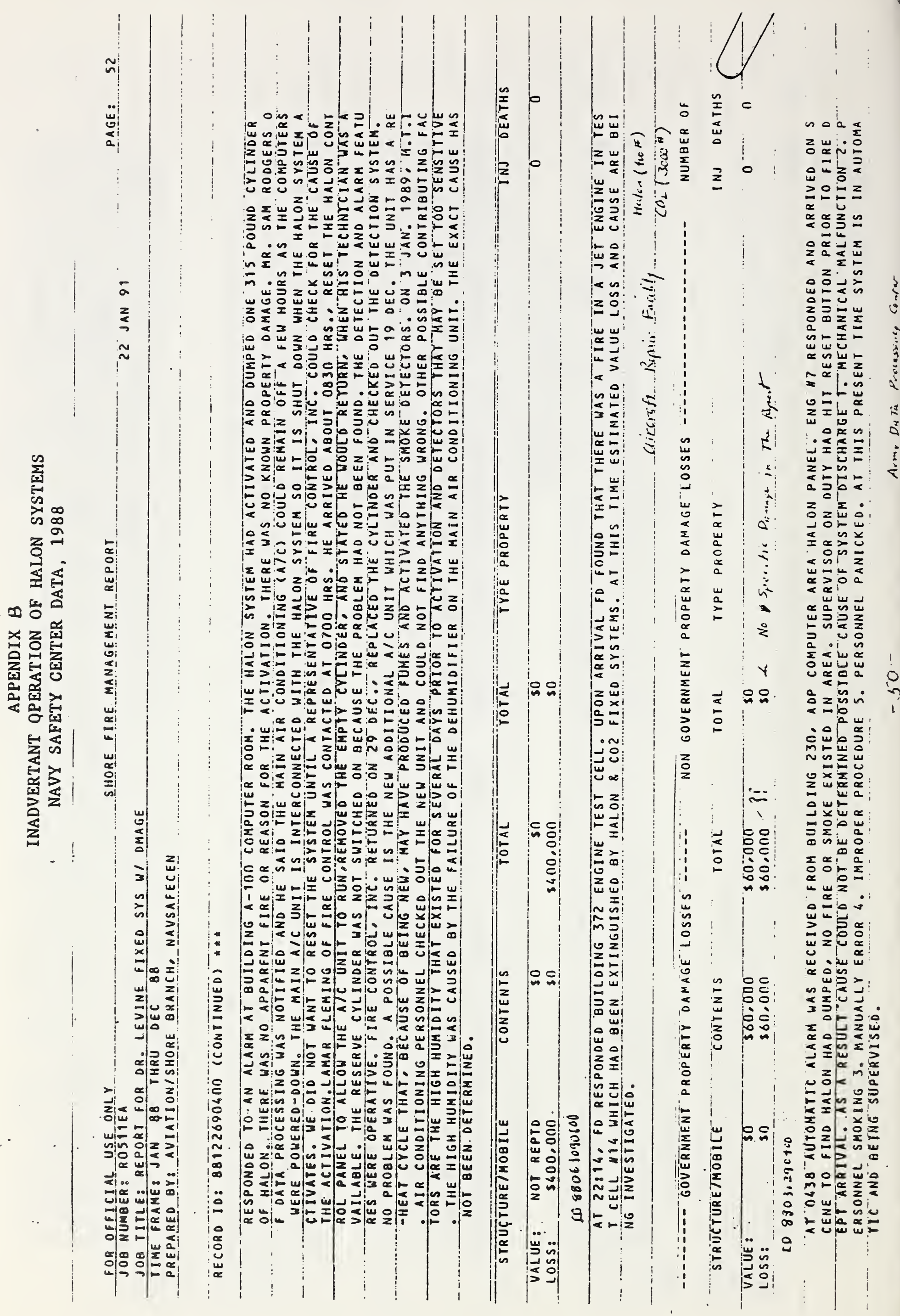




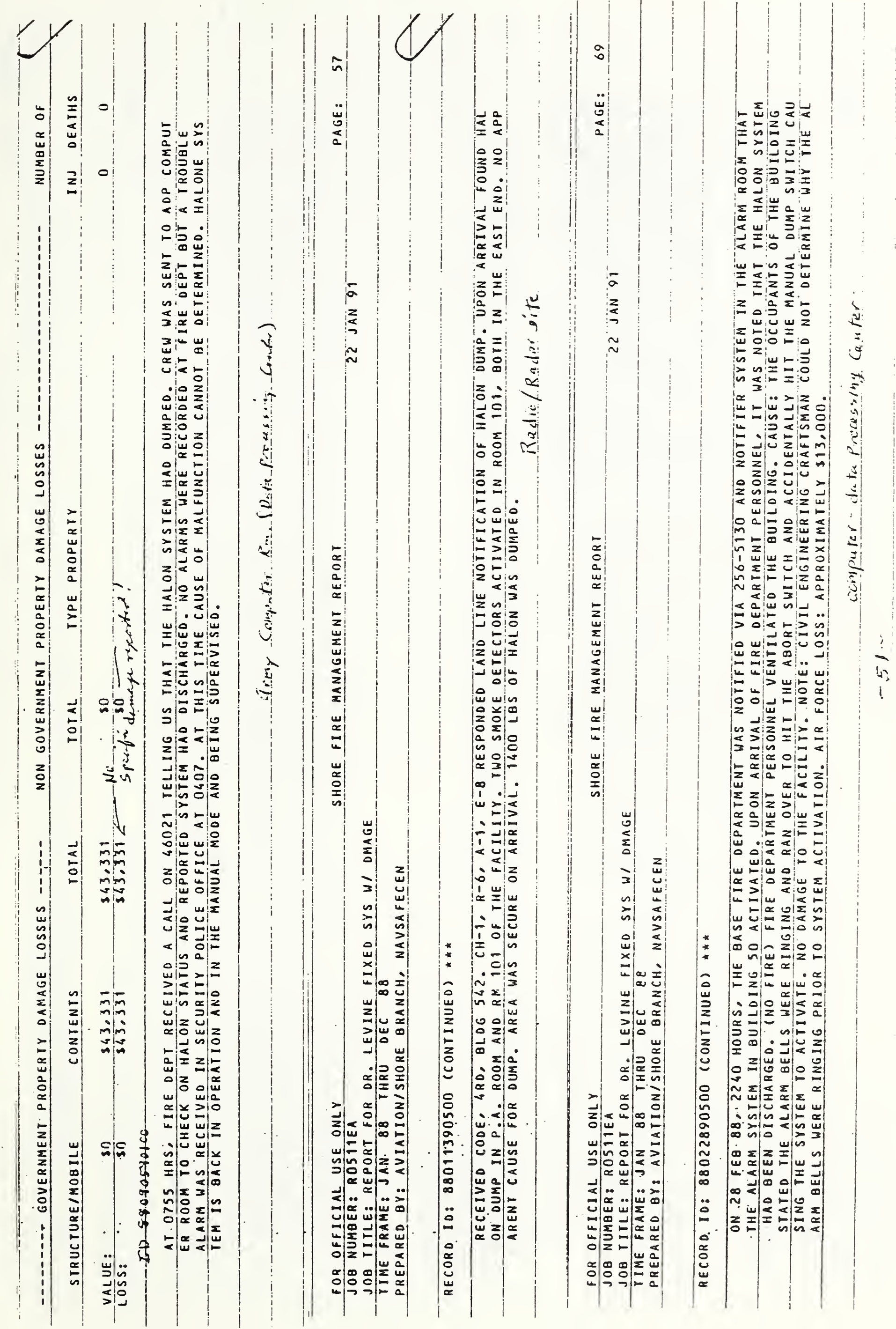




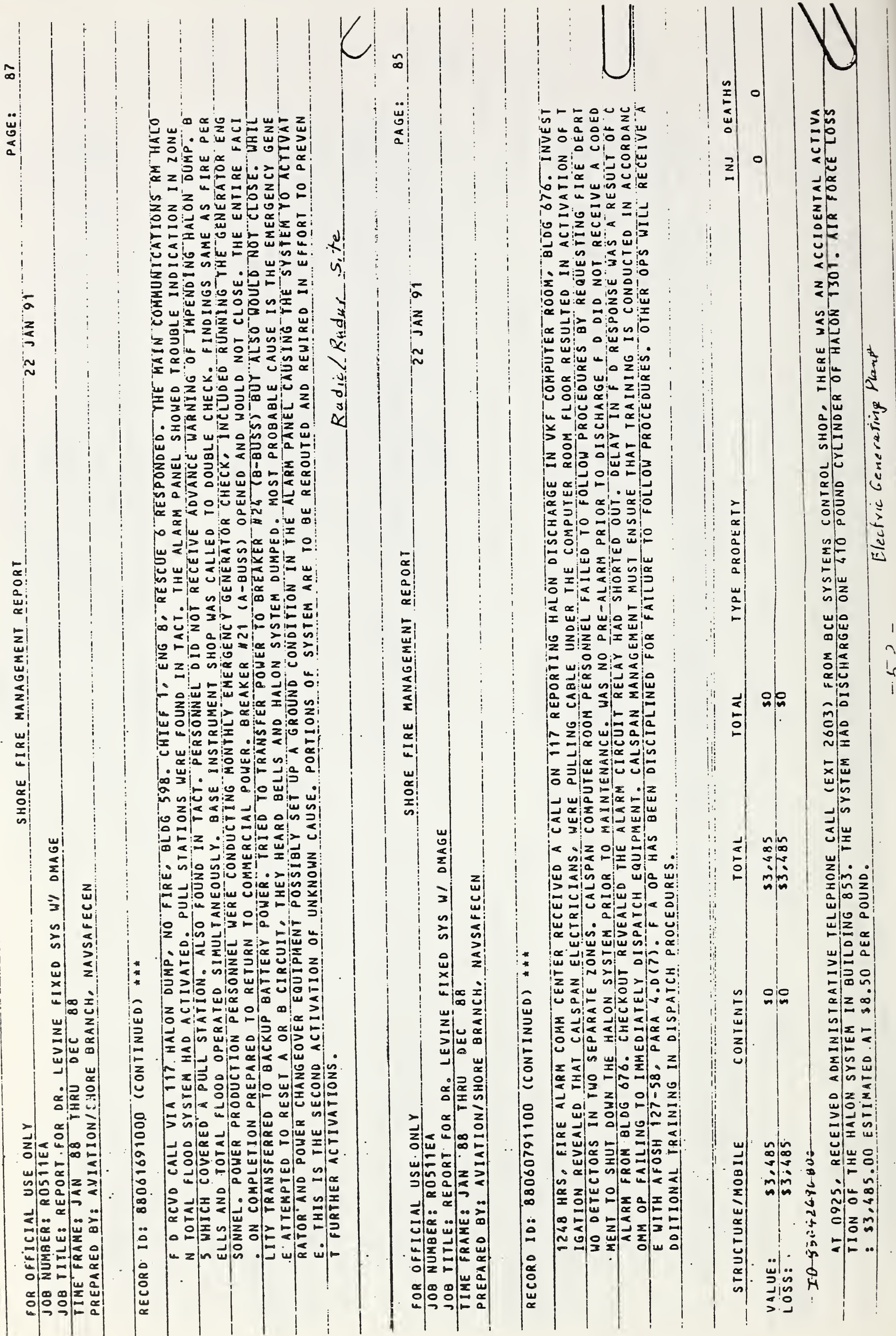




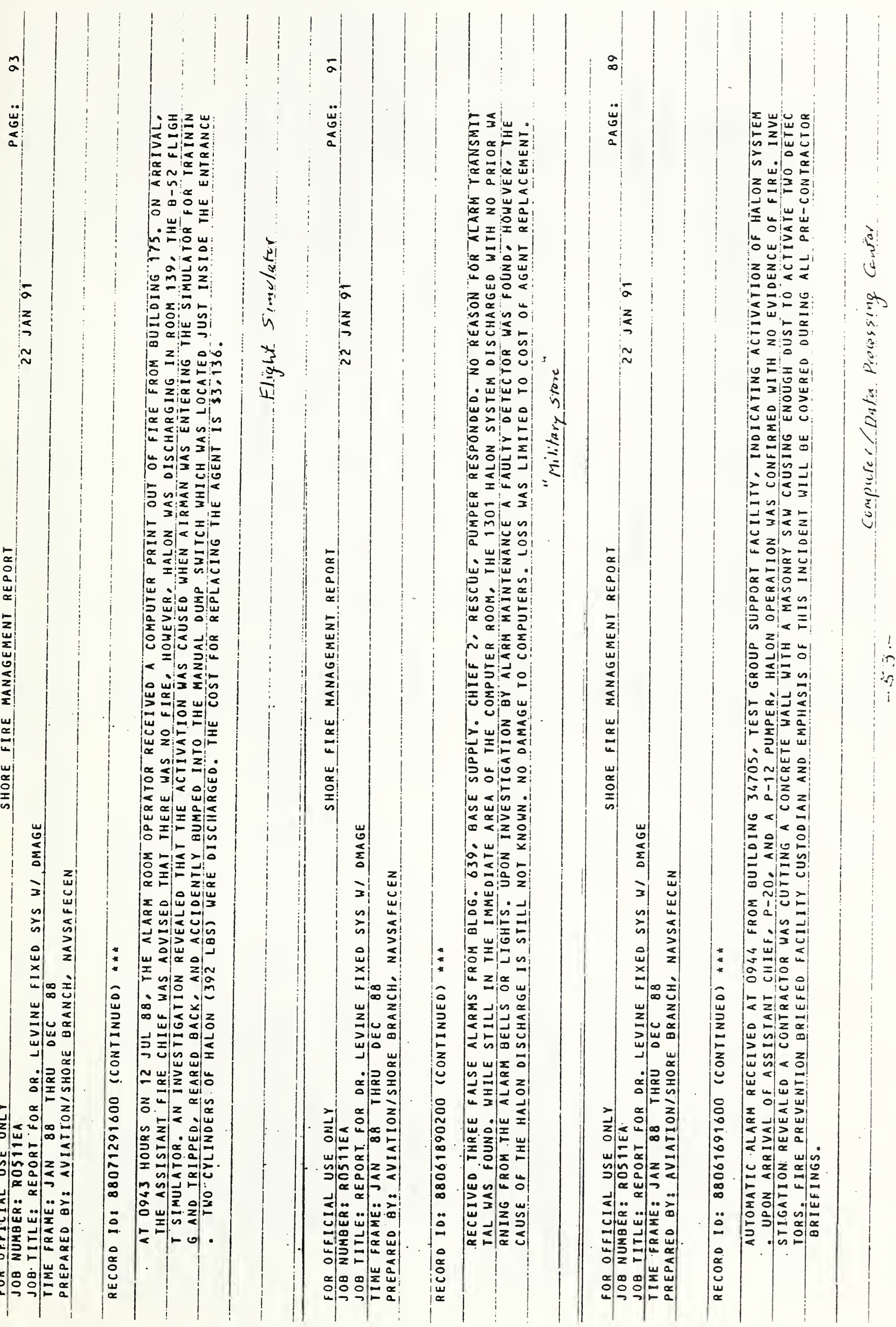




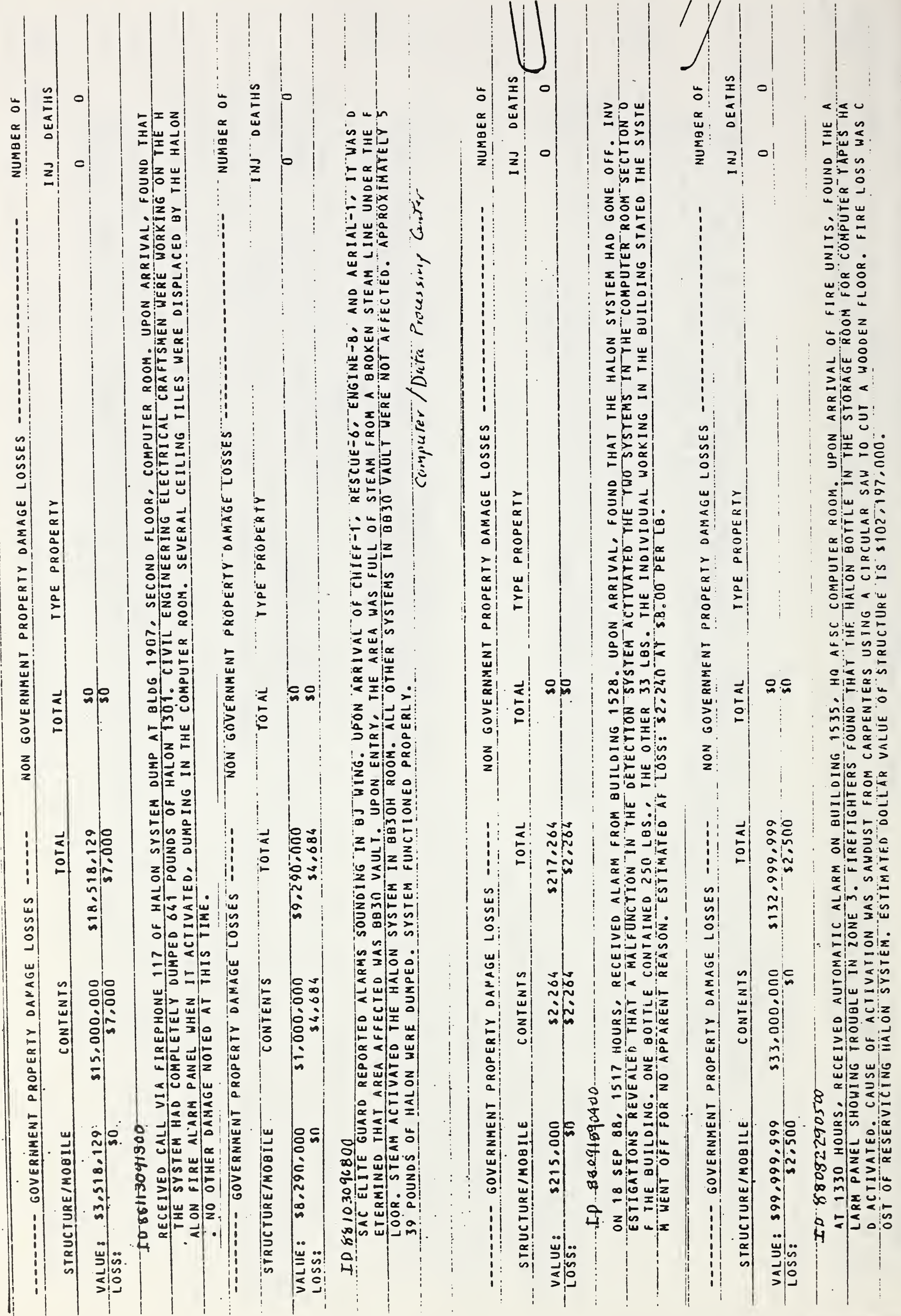




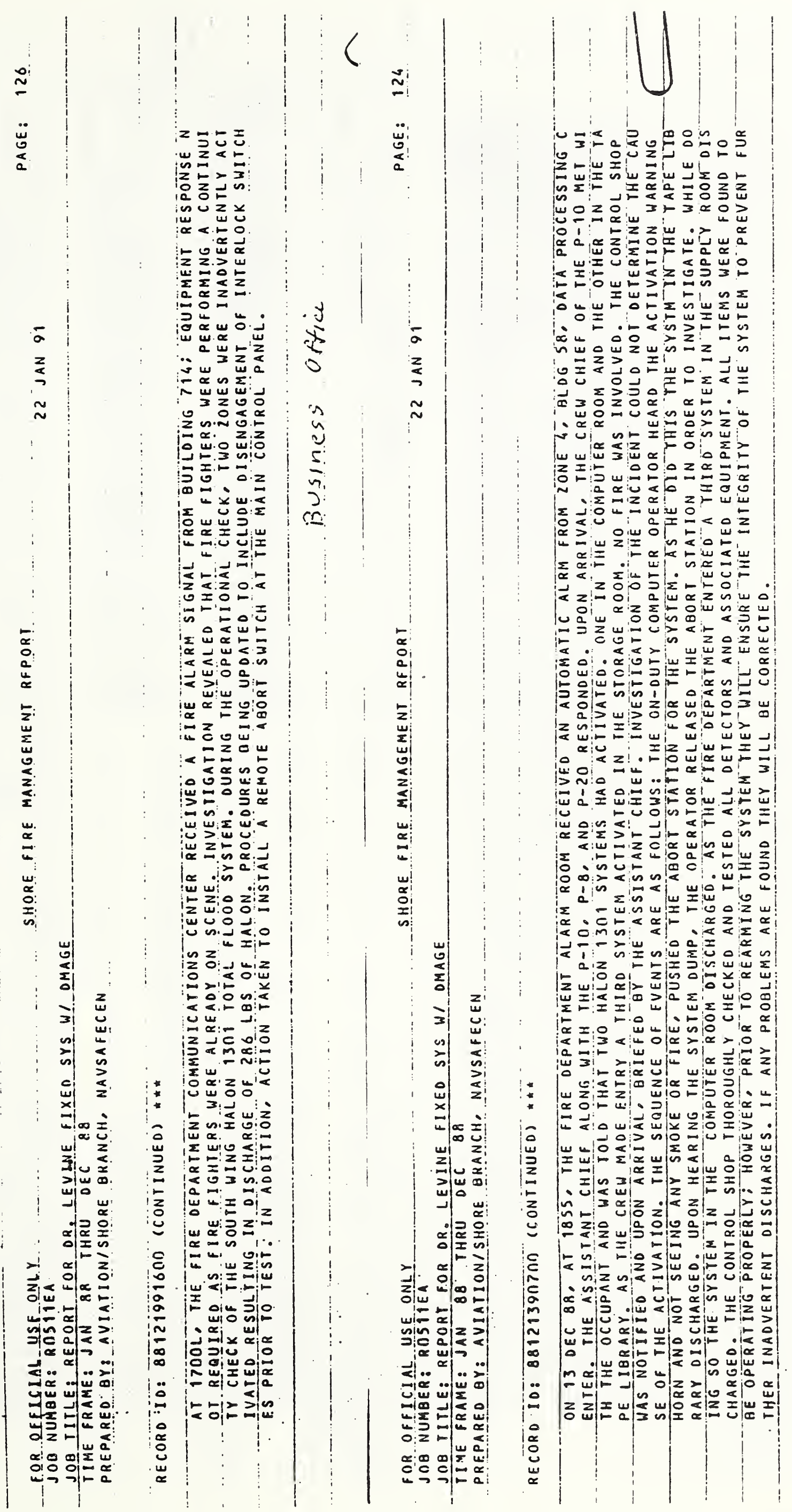




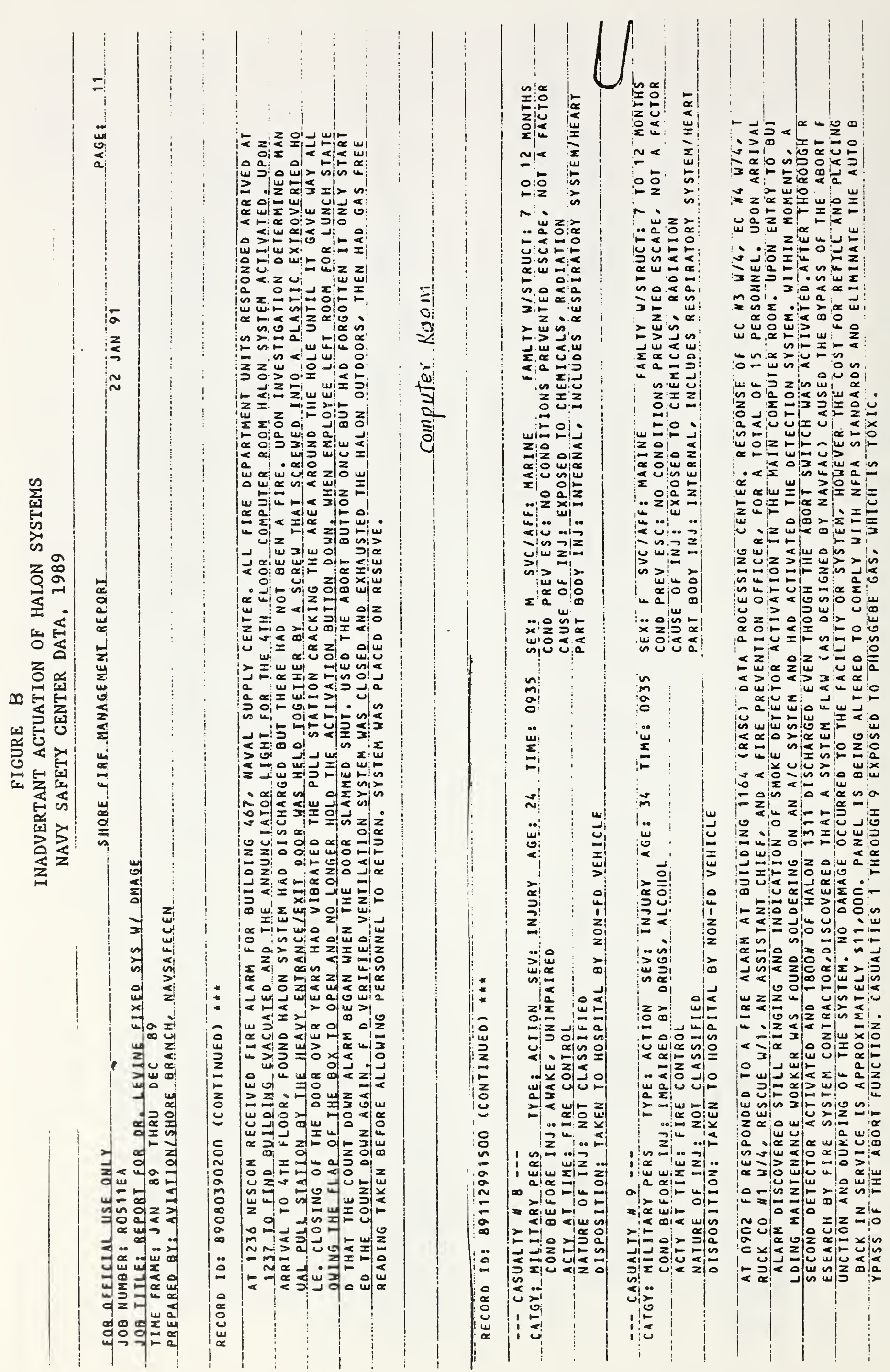




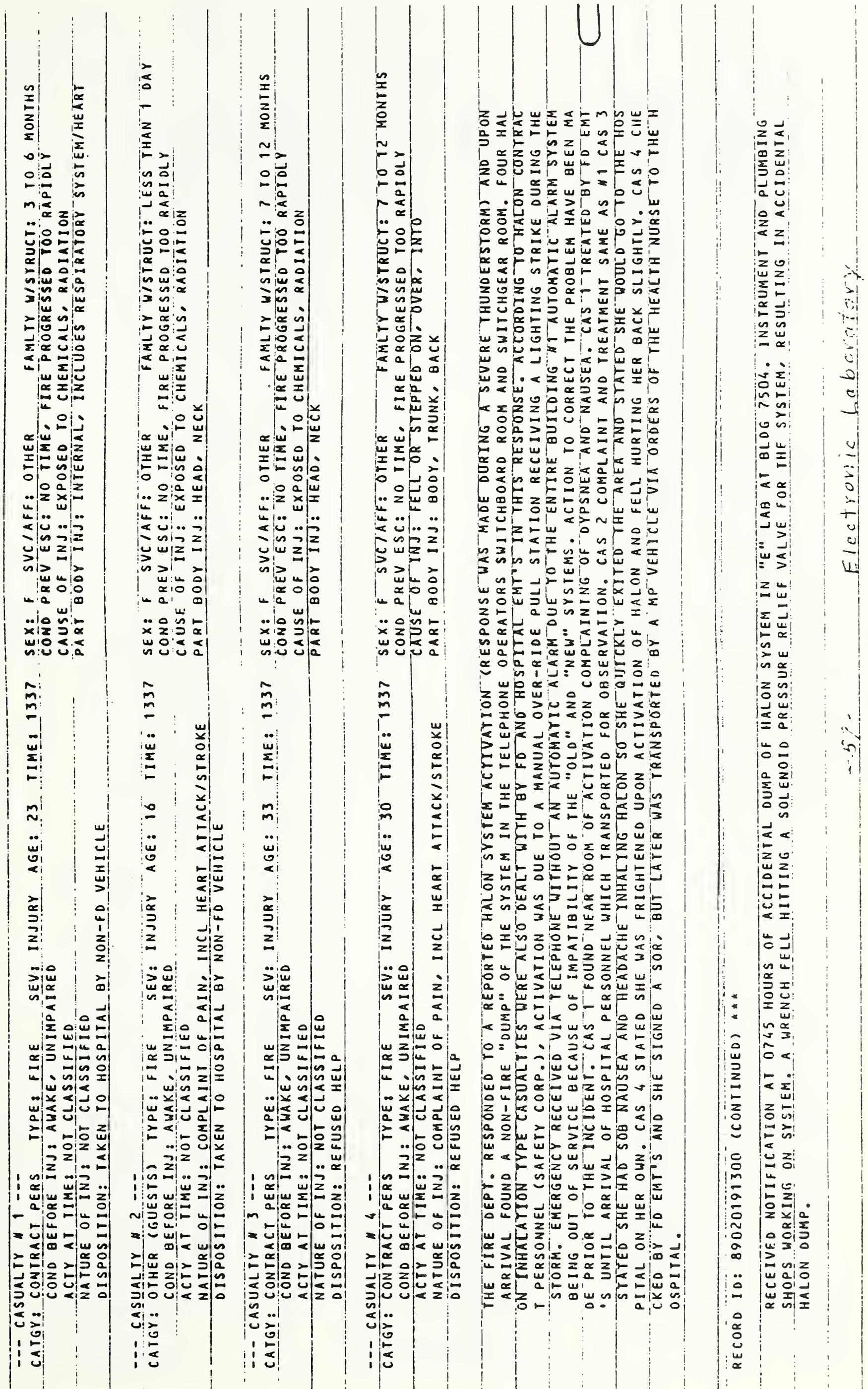



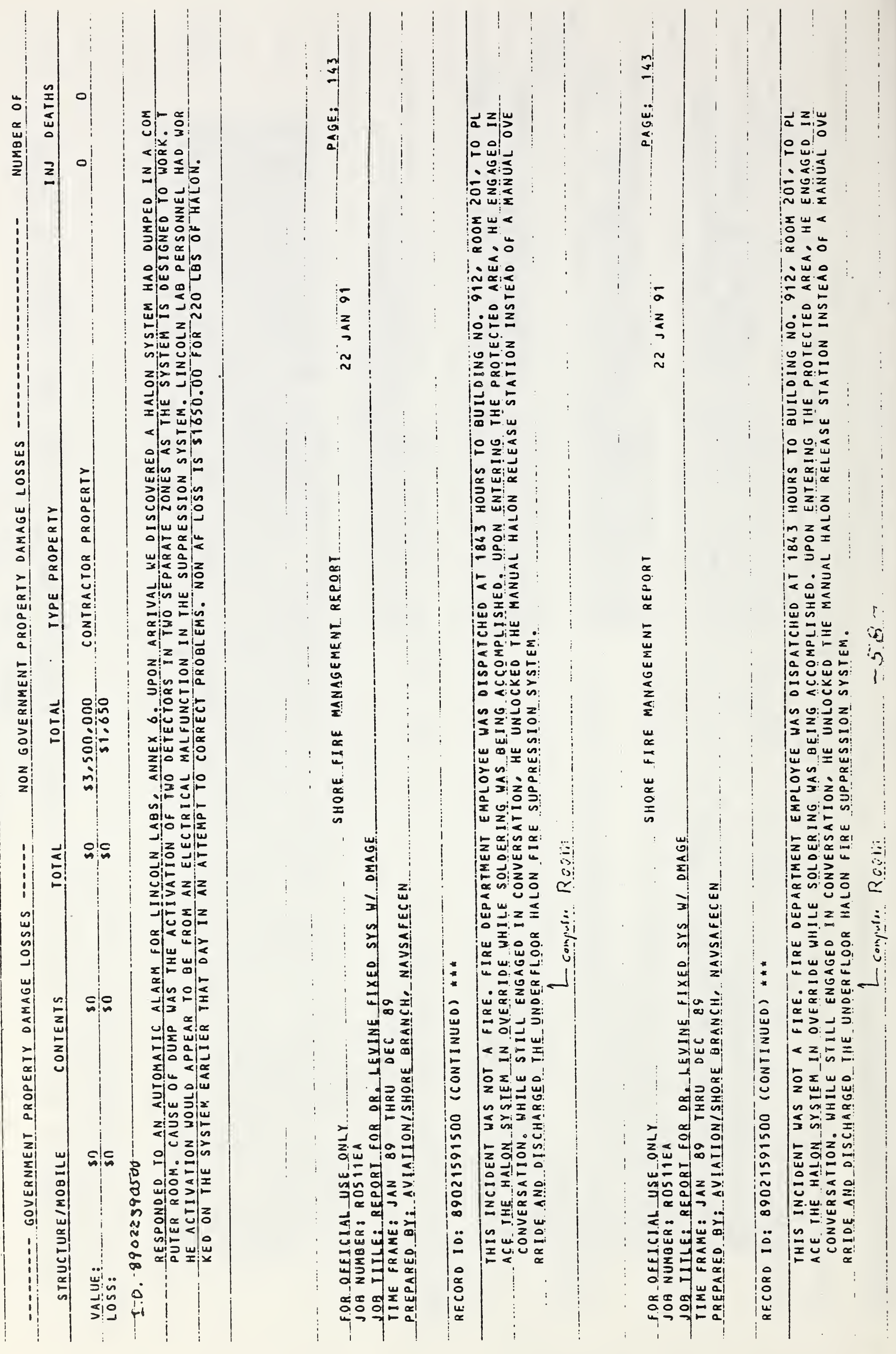


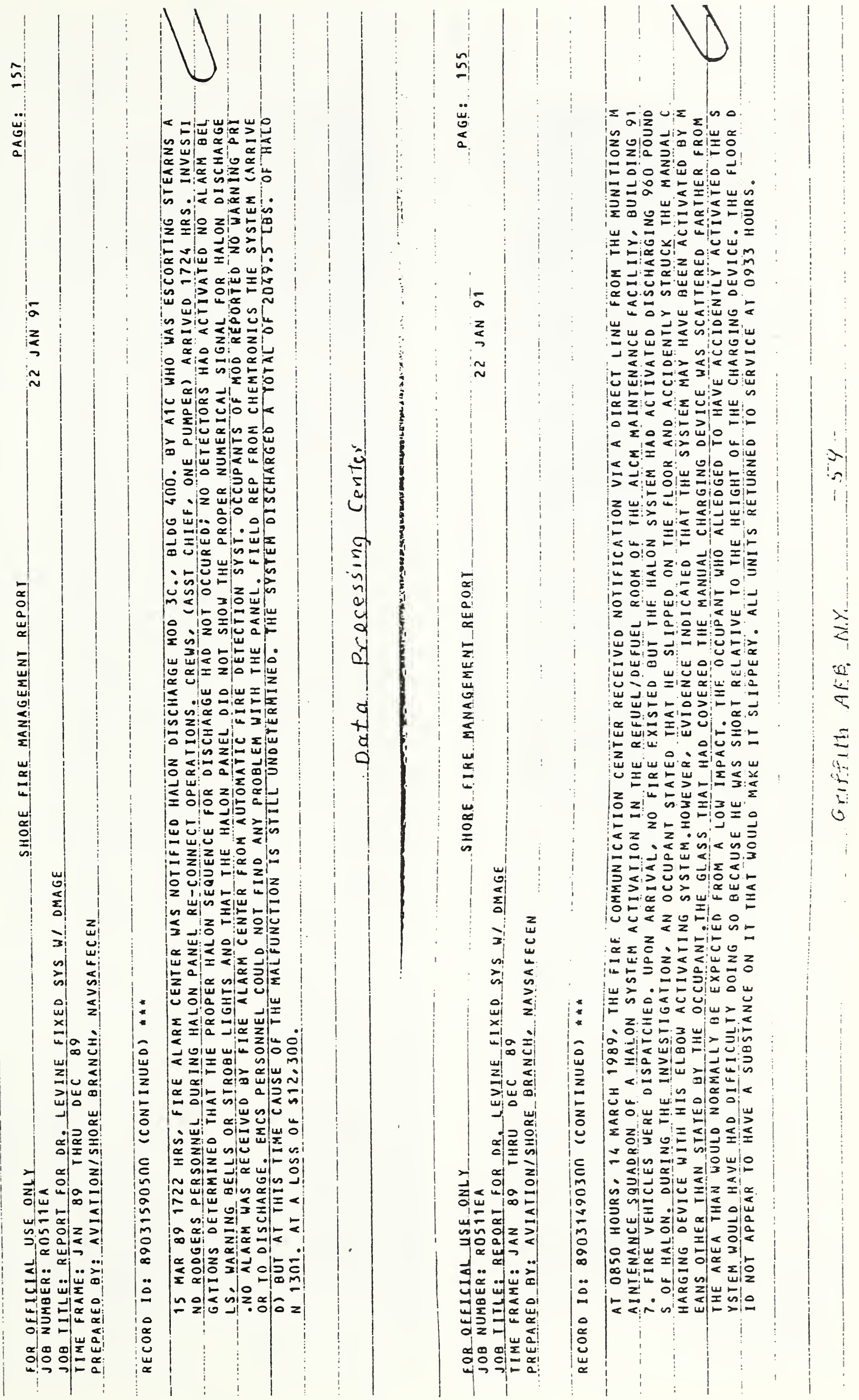




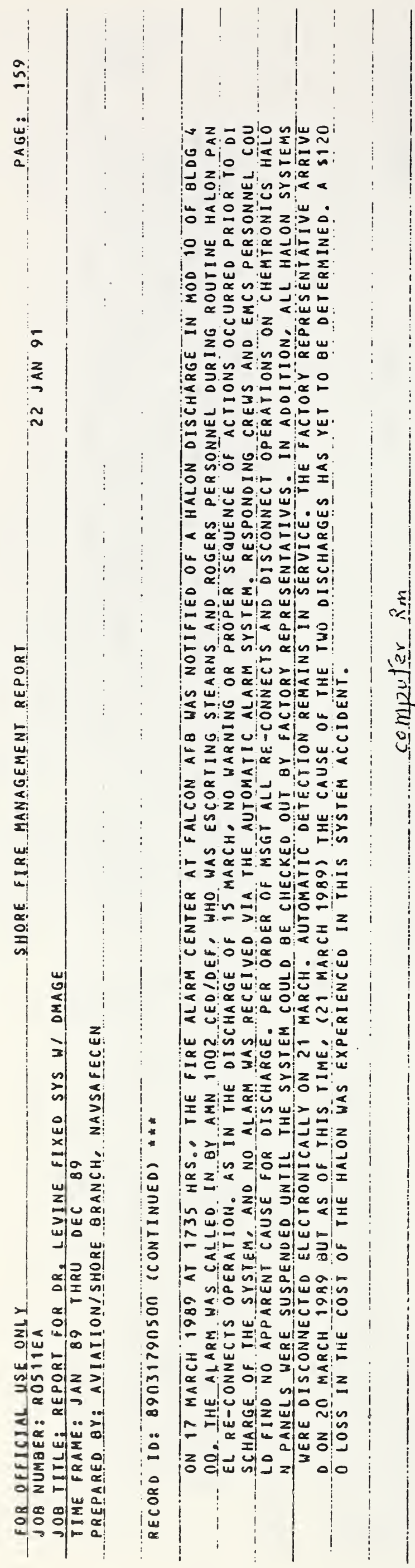

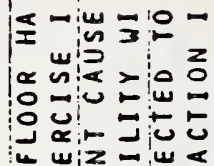

ه س

出 200

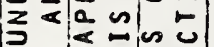

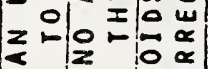

u

z

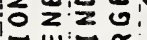

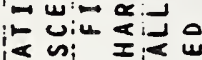

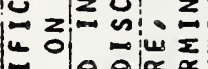

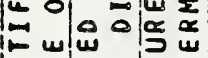

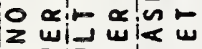

x:

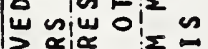

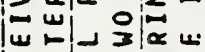

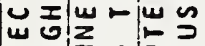

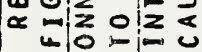

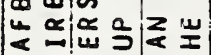

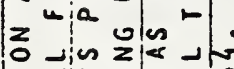

क

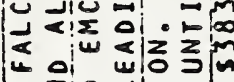

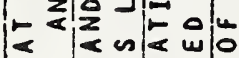

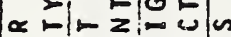

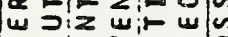

位

w 중 이잉

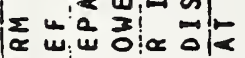

\&

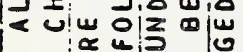

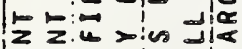

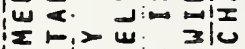

and

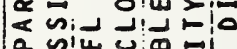

崖这 sion

u ma via

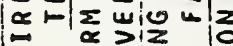

w 일

- F us un

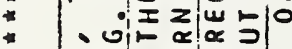

a

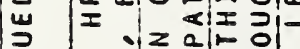

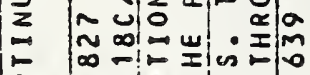

年

-

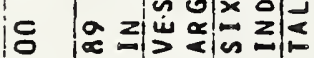

c

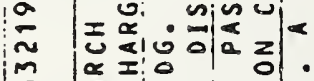

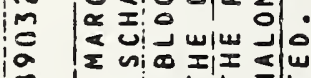

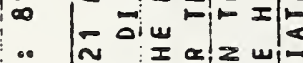

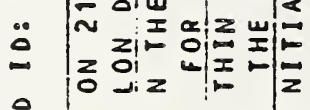

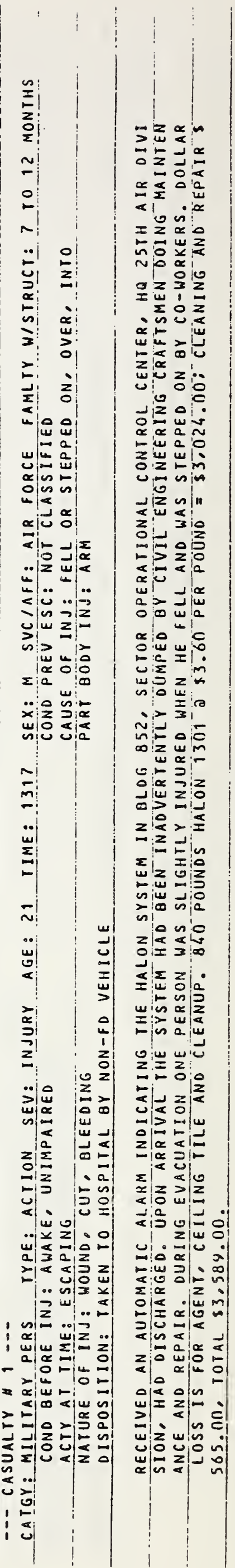

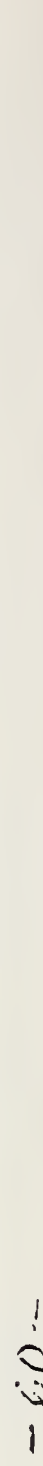

.

!o

. 


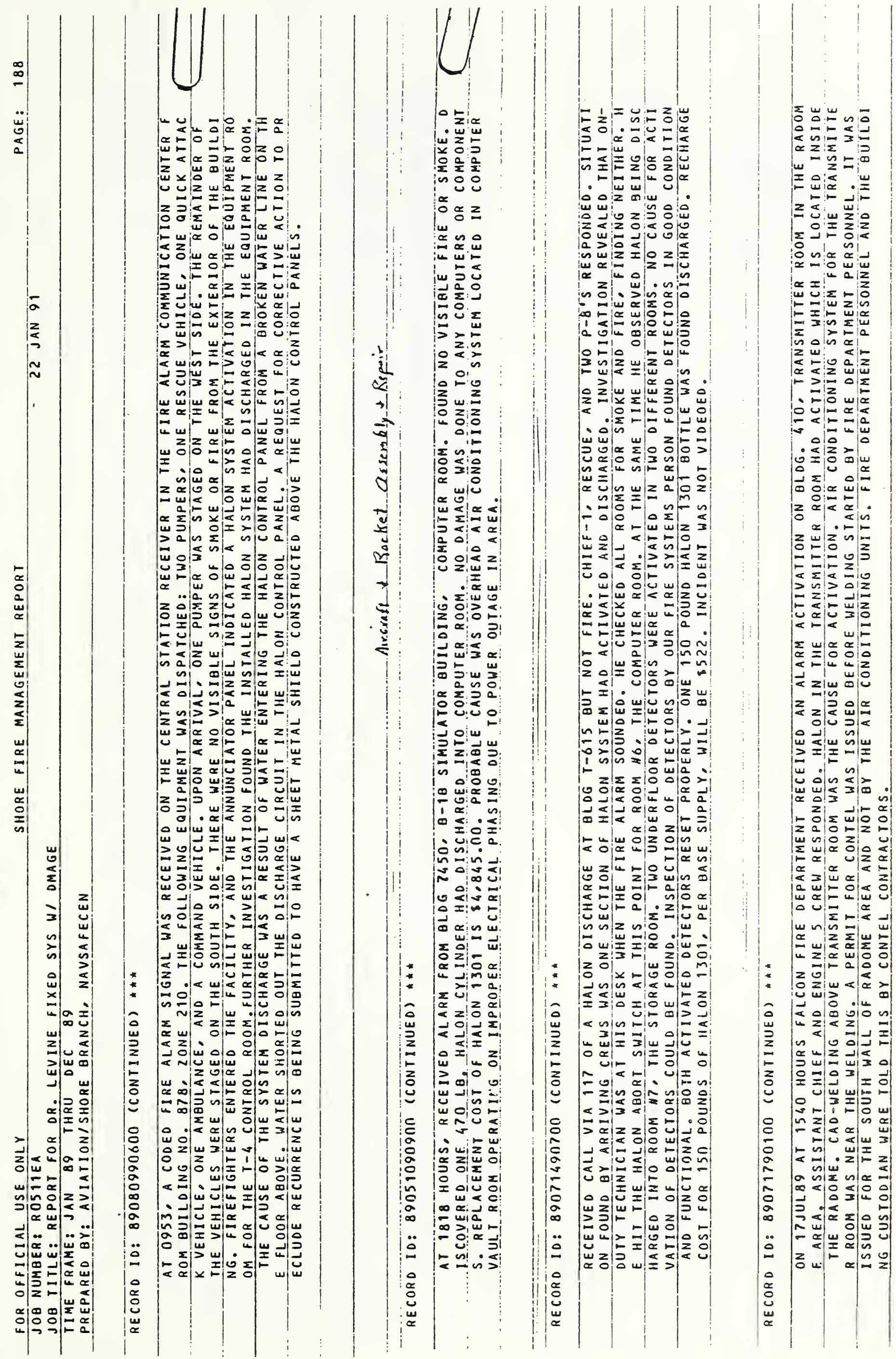




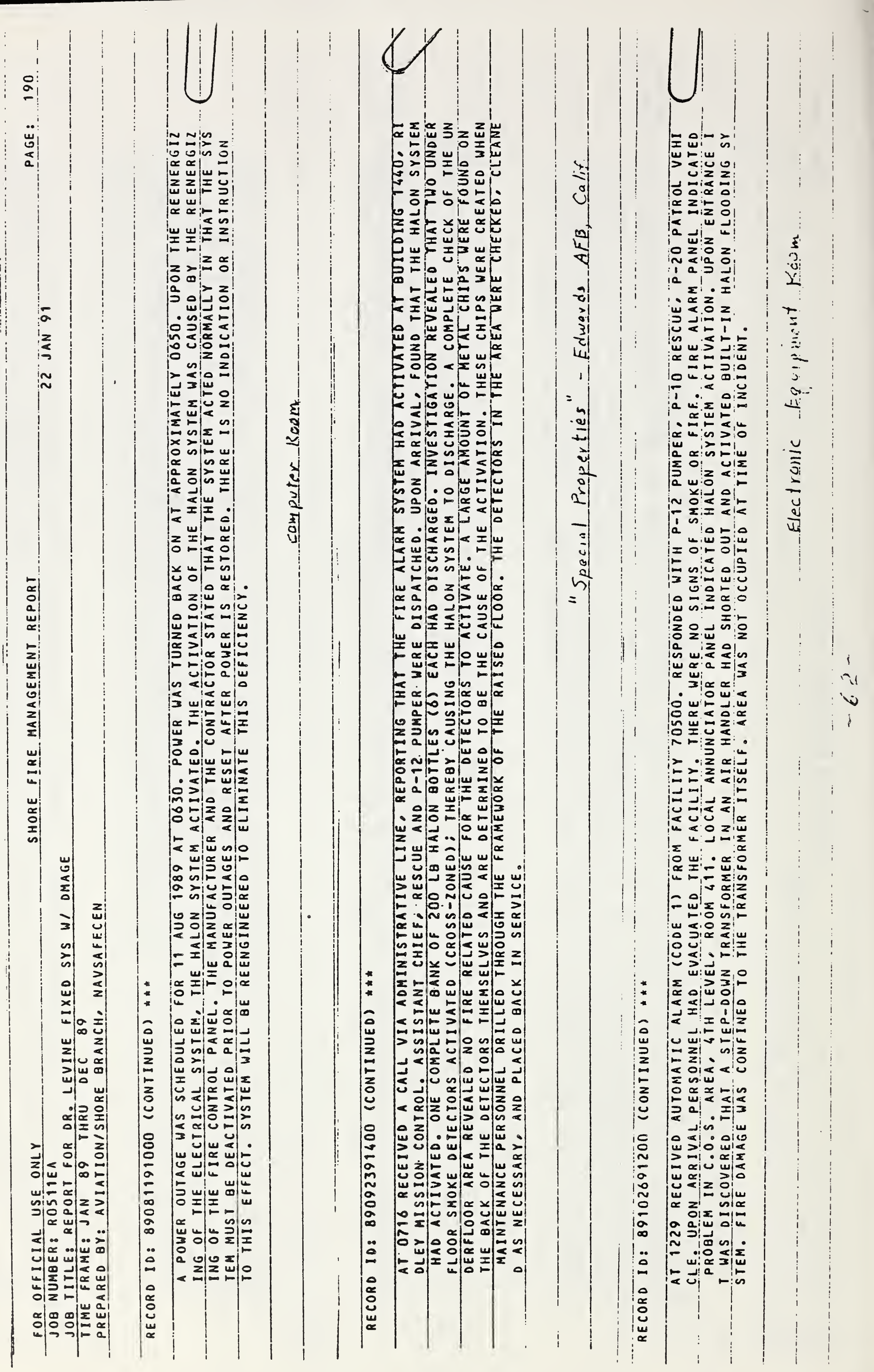



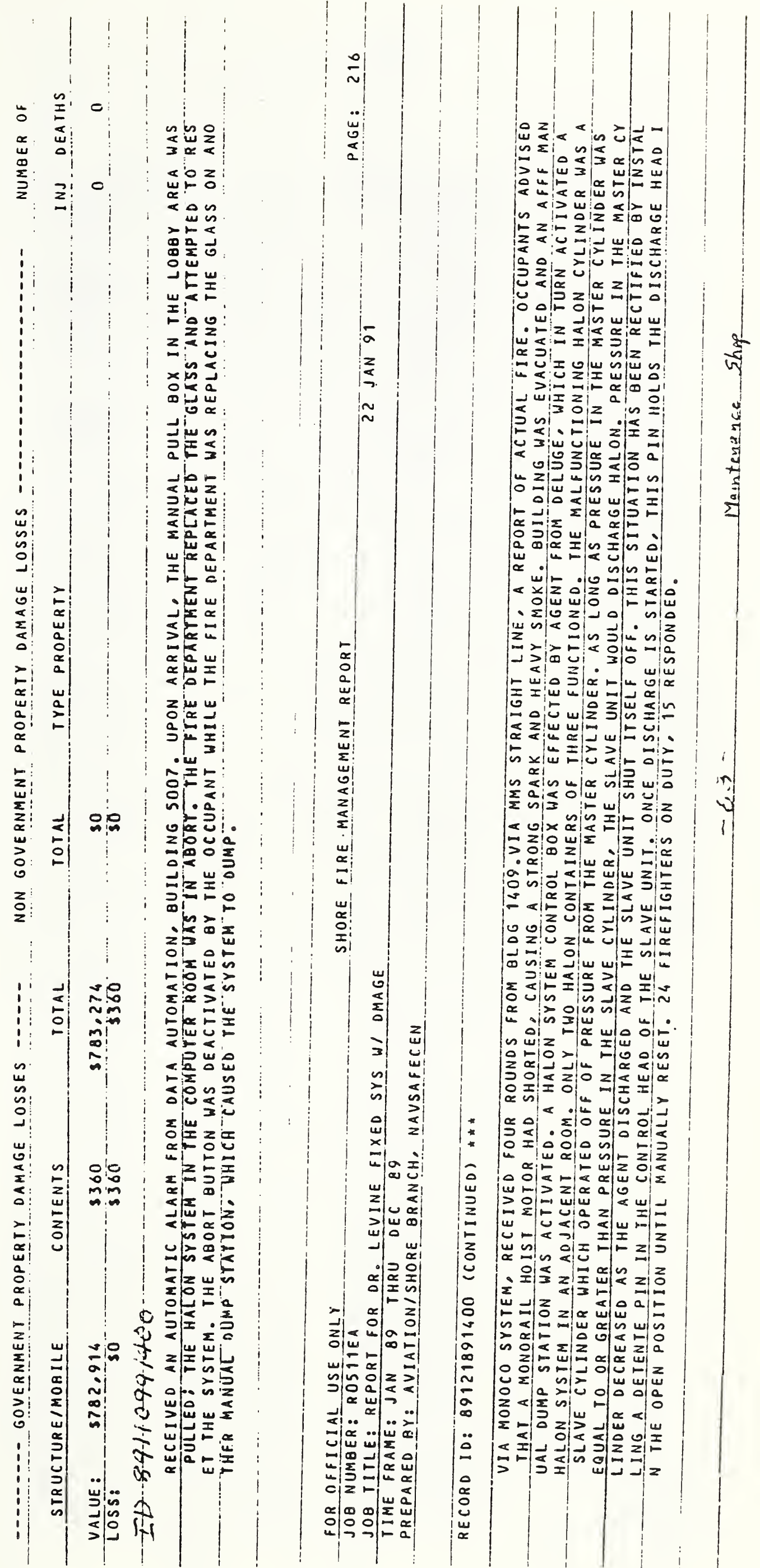


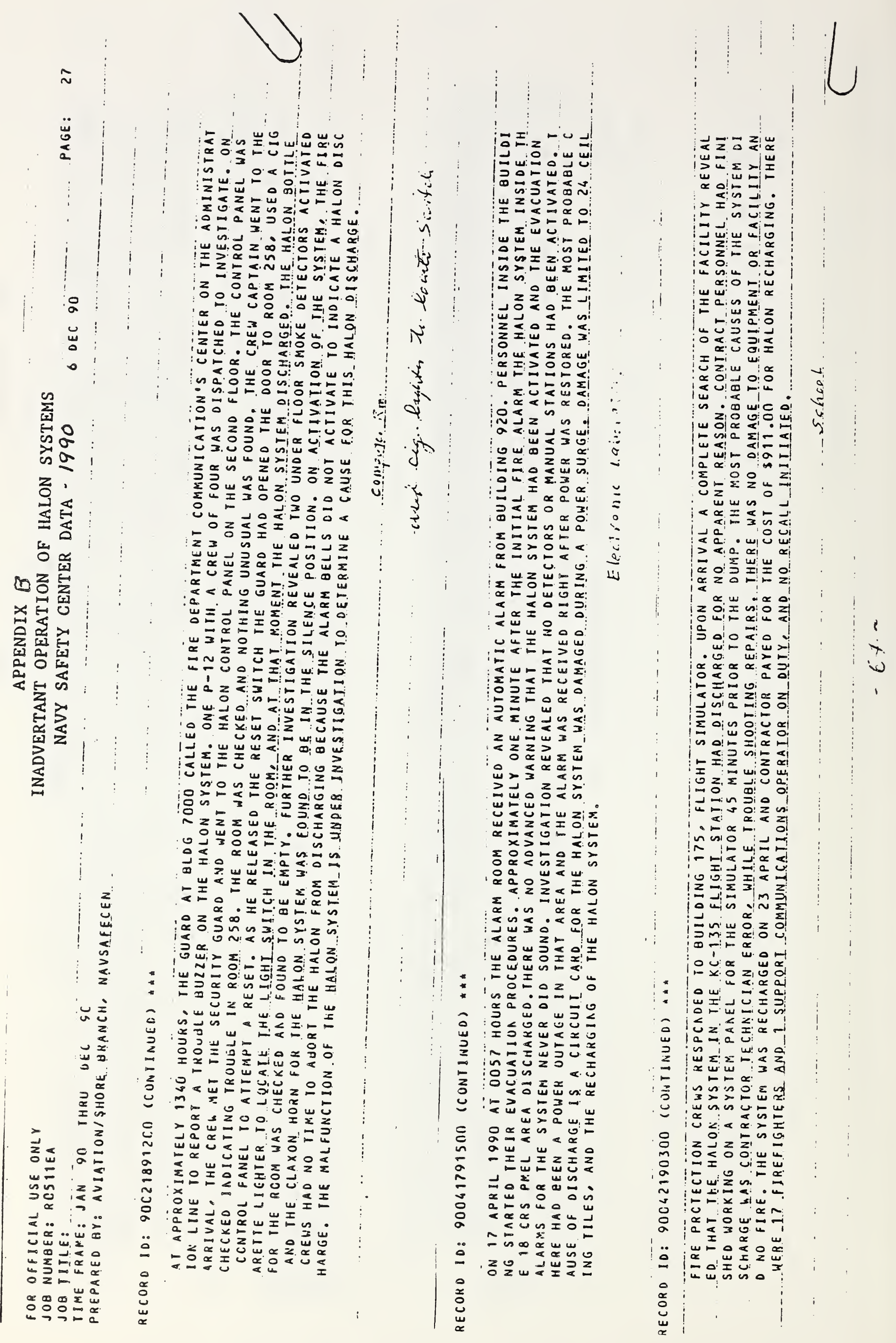




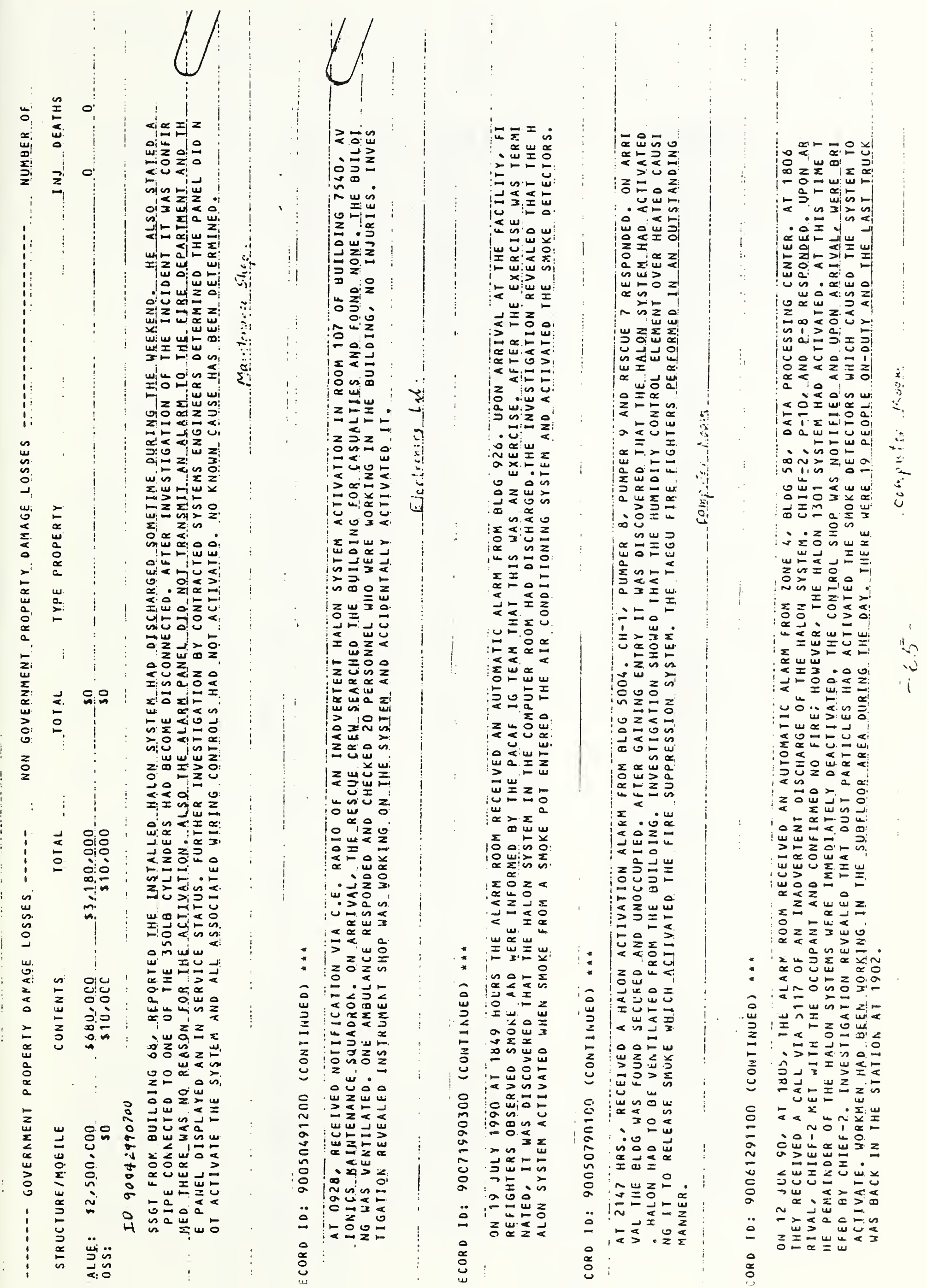




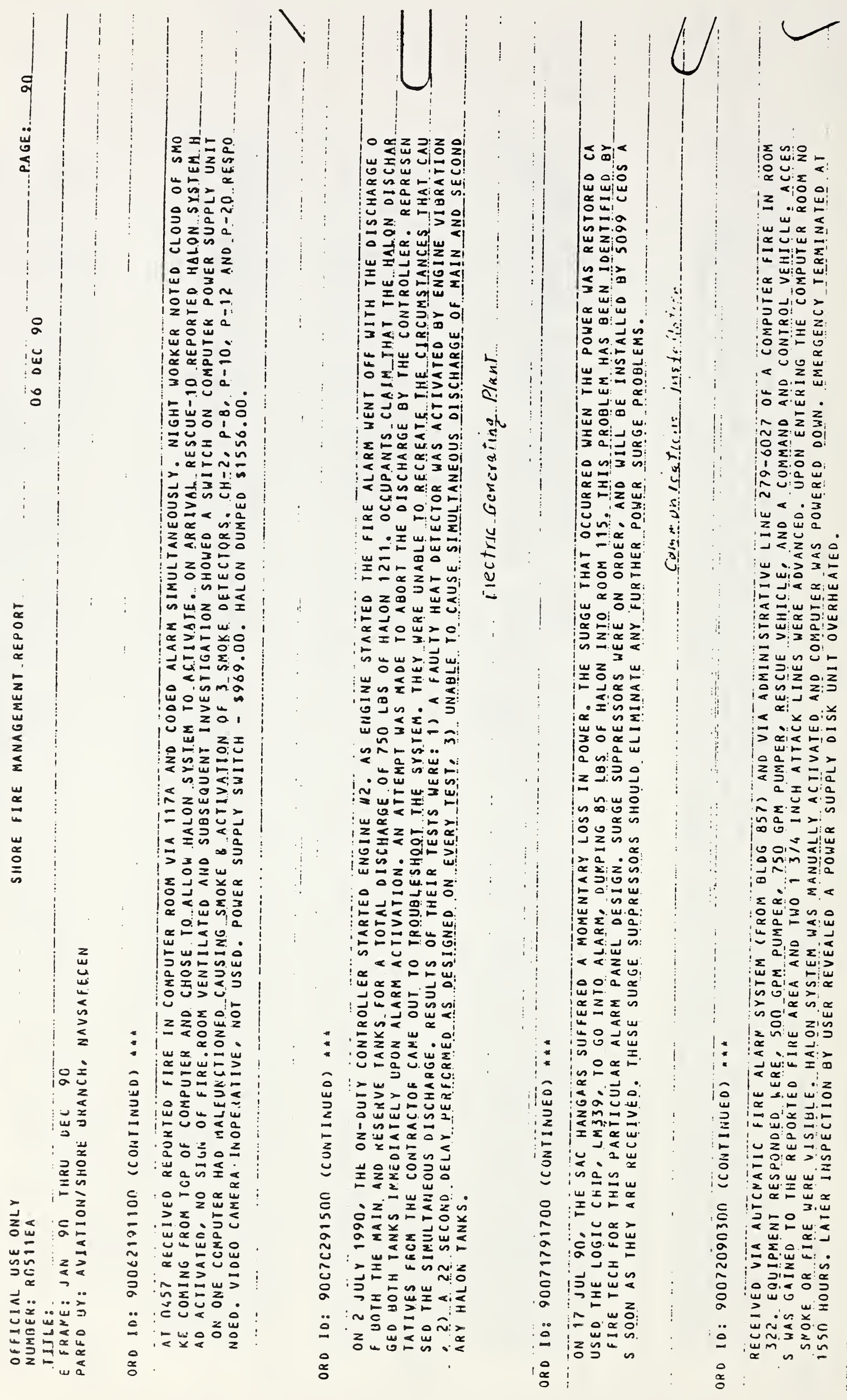




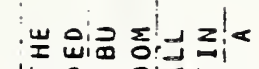
.

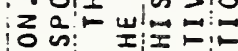
wo w他

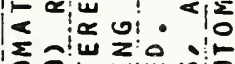

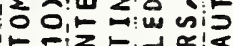

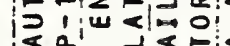

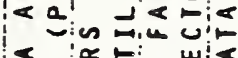

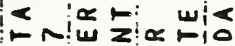
í w

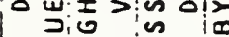

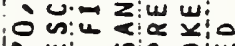
مّ

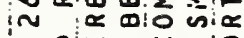

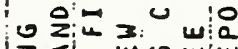

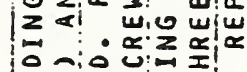

귕 w

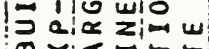

> x $0<2$

-

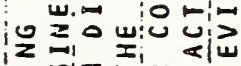

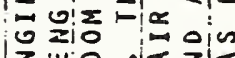

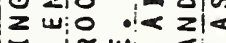

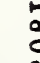

告

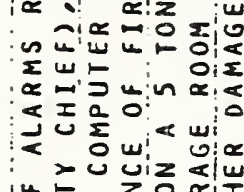

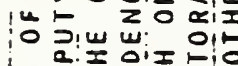

z

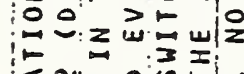

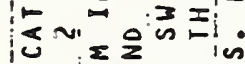

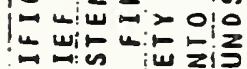

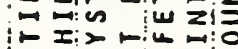

o vin o:

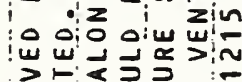

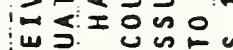

记出

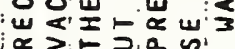

|:

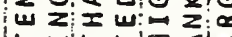

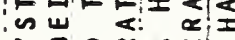

is

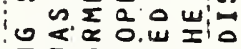

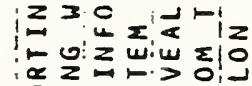

ion 0 ind

:

की

iv

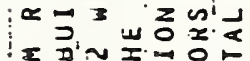

:

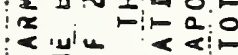

공오

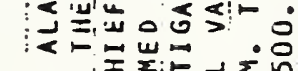

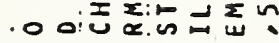

z

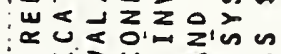

0 a $000<00$

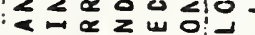

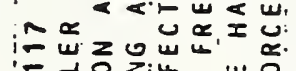

- -

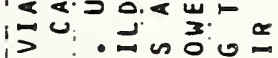

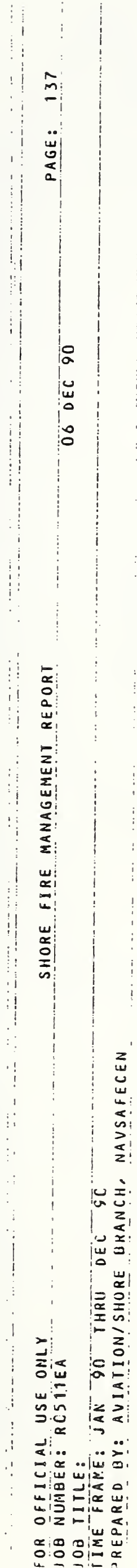

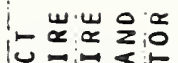

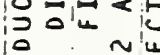

준ㄷㄴ

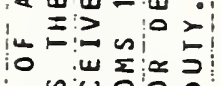

10 4u no잉

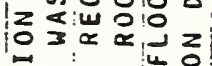

清・边

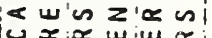

는

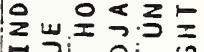

$\leq$ 岁央<冓

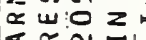

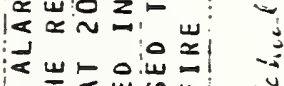

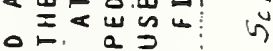

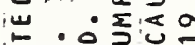

a

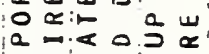

証之次

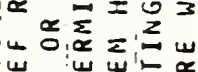

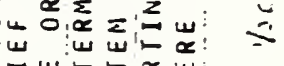

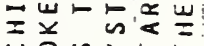

足进的的。

3 的 3

wo>0 $2 \sum \sum_{w}$

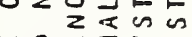

닌요

品

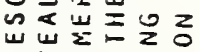

1.

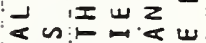

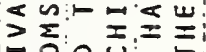

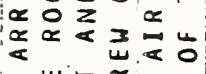

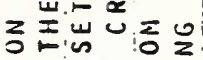

م

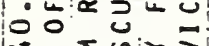

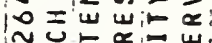

N

$0<2 w=w$

w

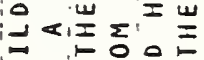

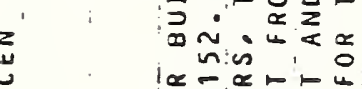

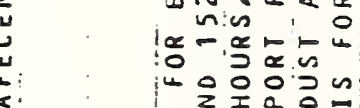

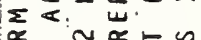

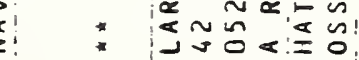

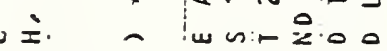

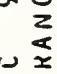

㟧

峁

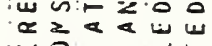

治:

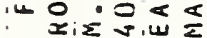

10 w口:

$\omega_{2} z=0$

un

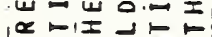

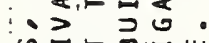

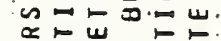

氠岕的全

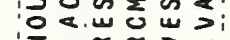

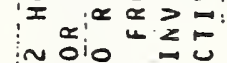

이일

识出这的。

$\ddot{a}$

i出造的。 


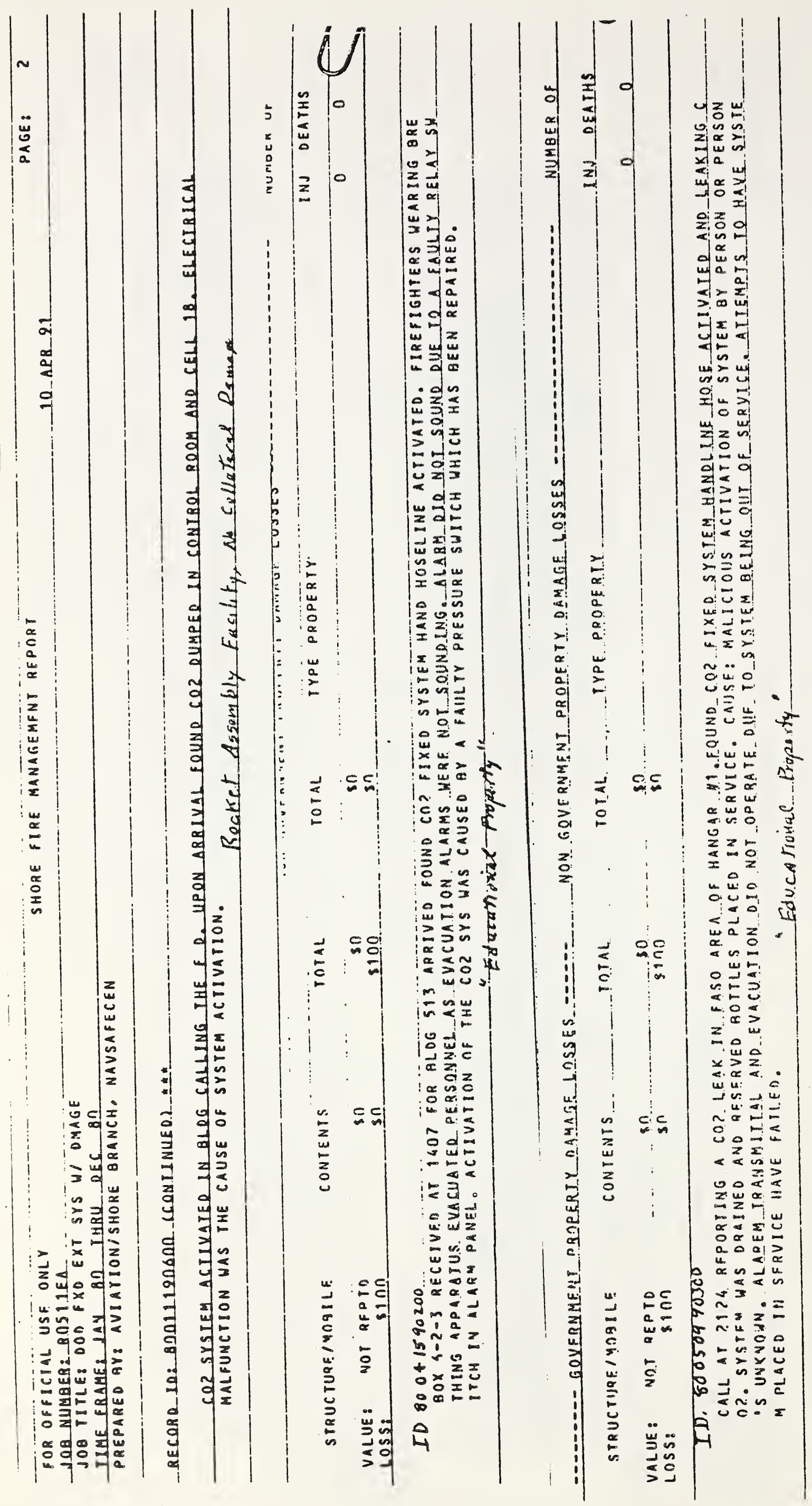




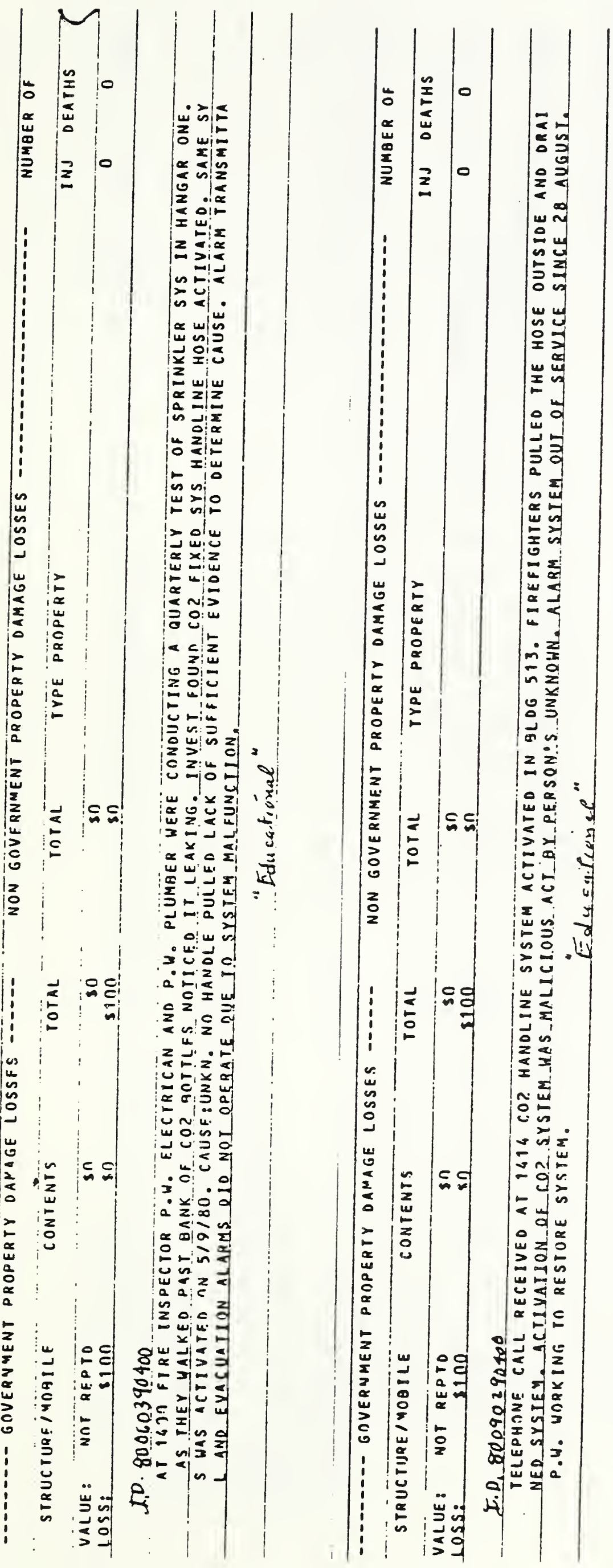




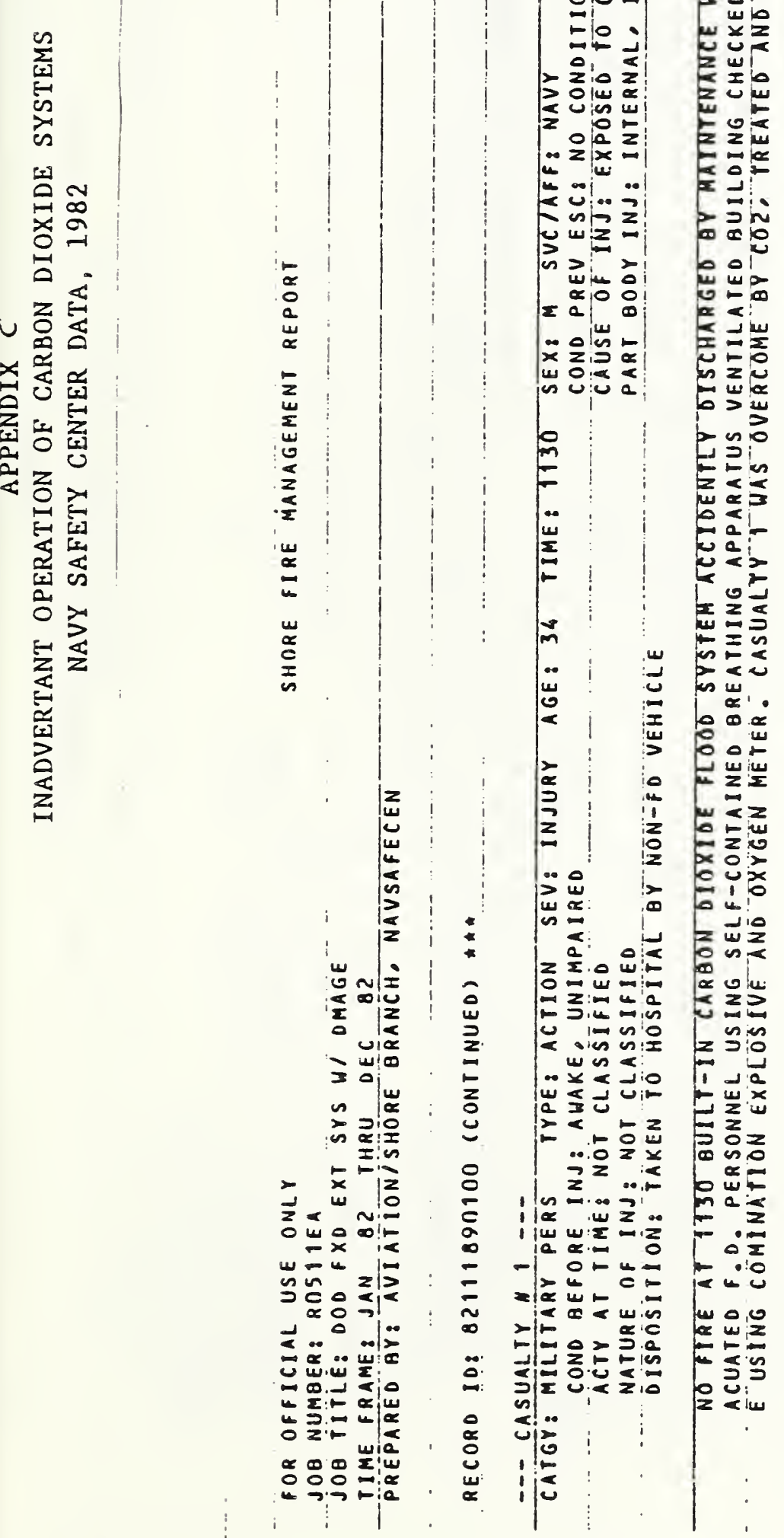




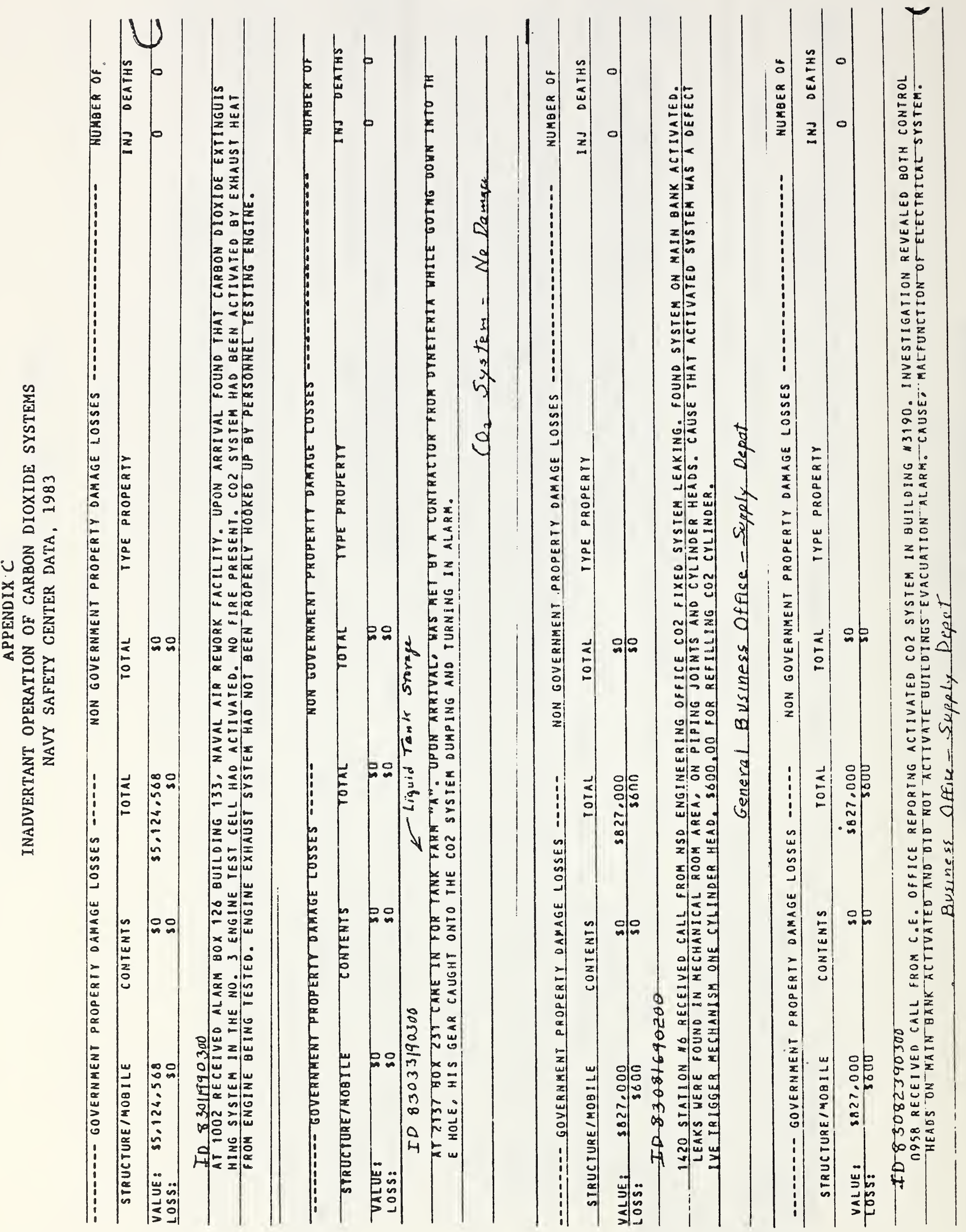



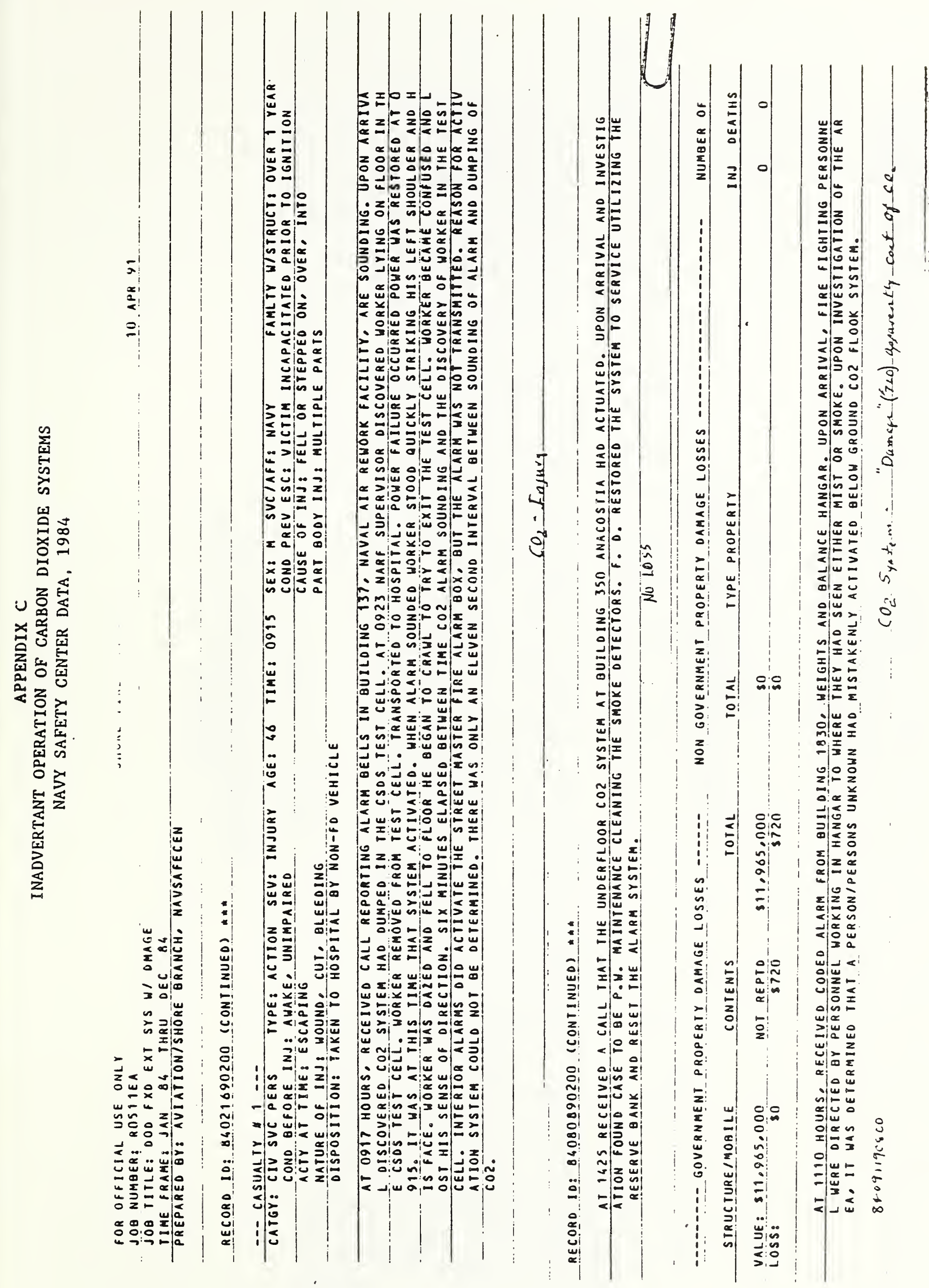


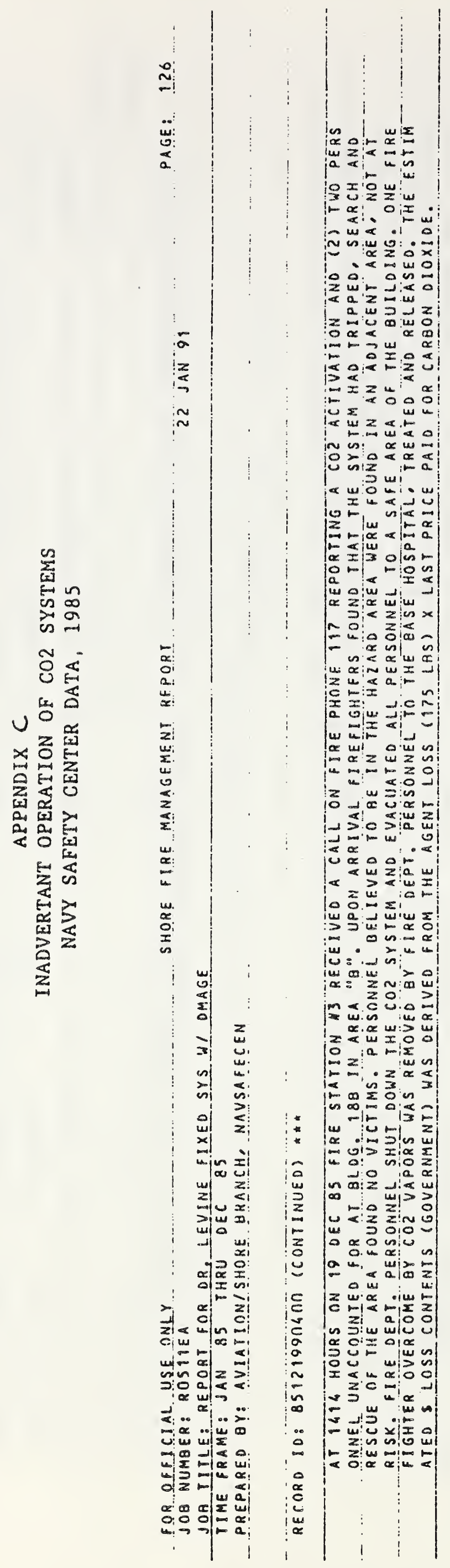




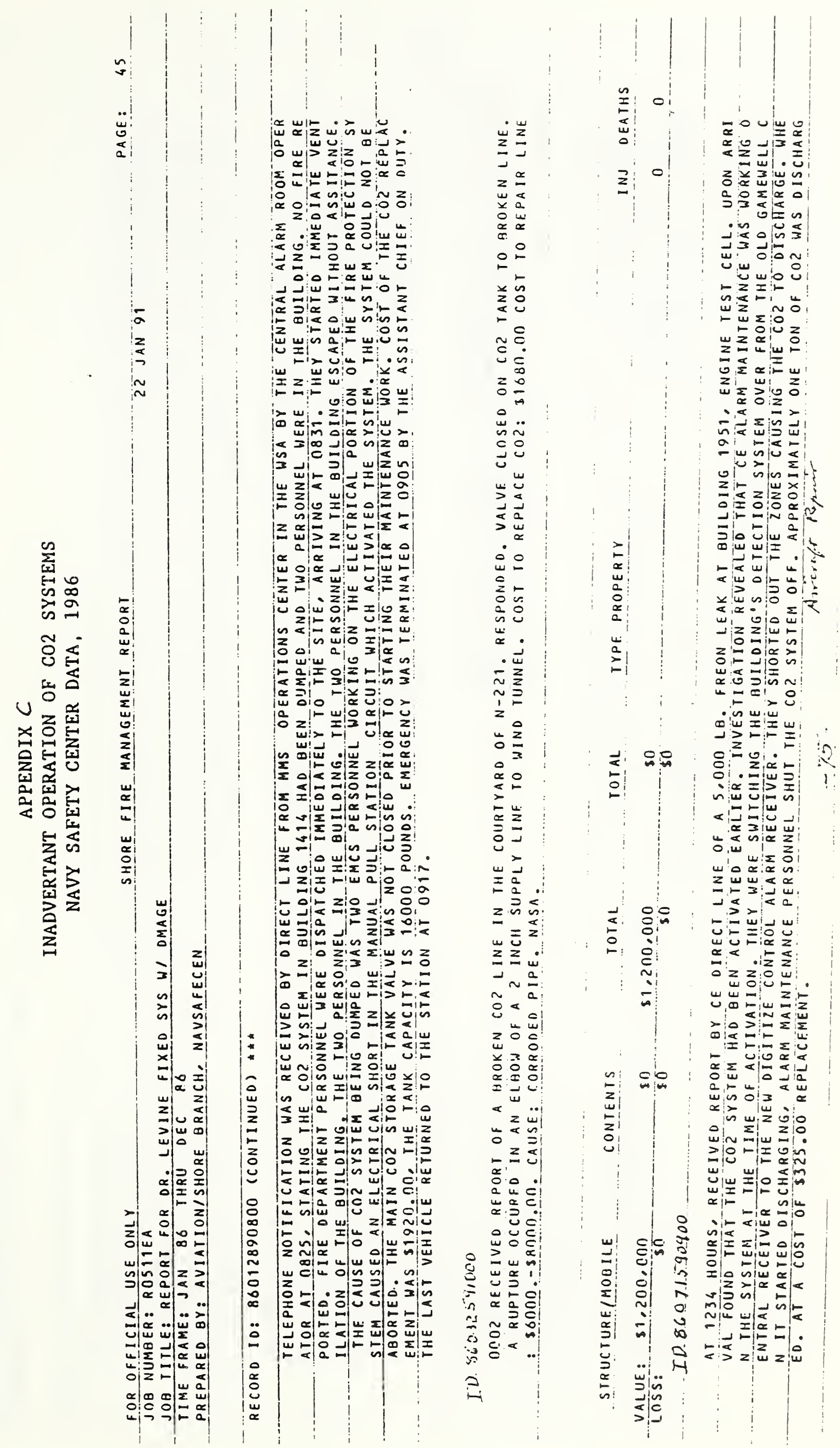




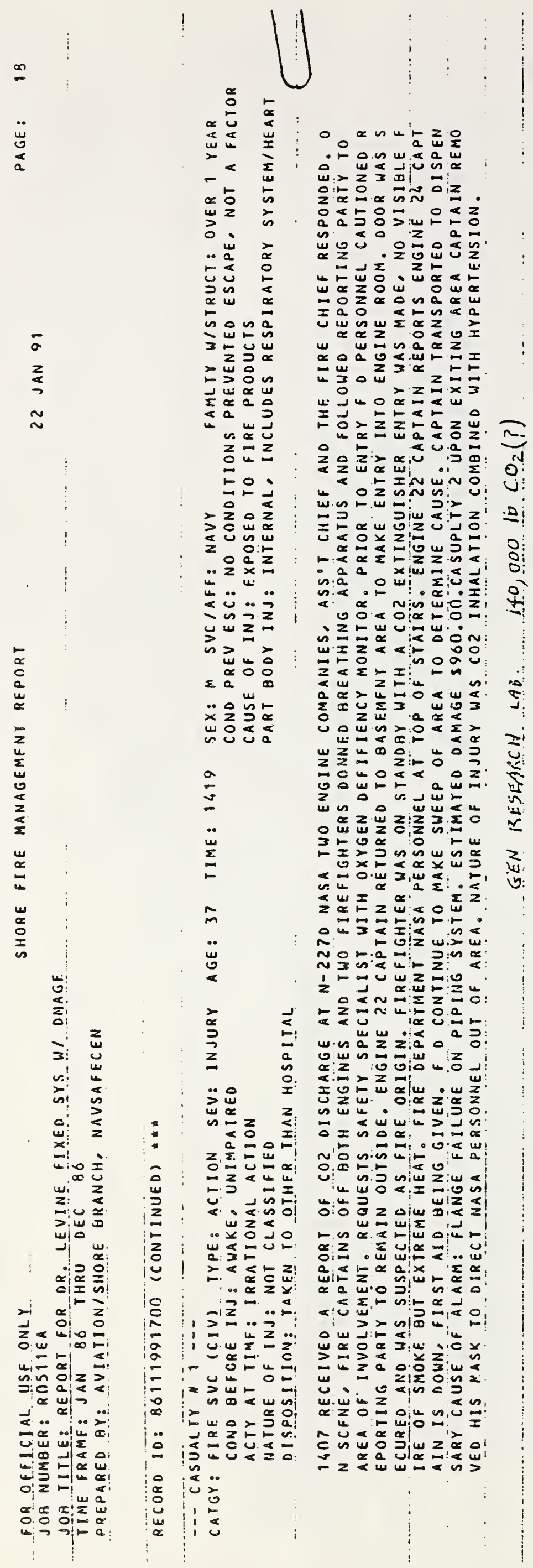




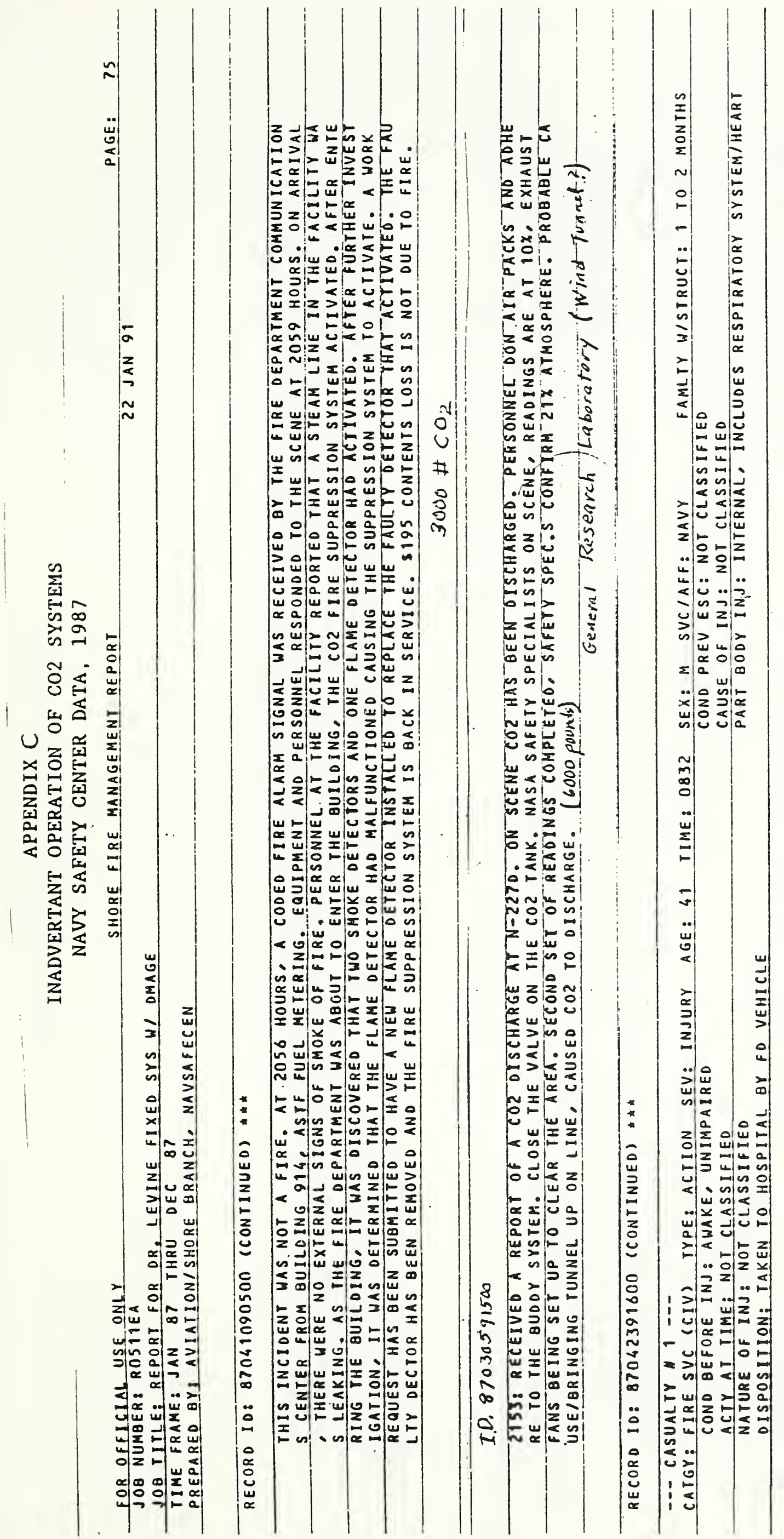

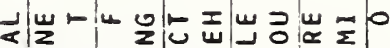
ว

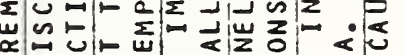

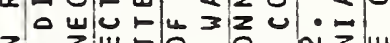
z, 2 z出

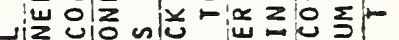
山اz

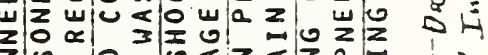

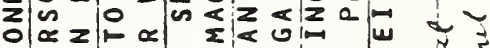

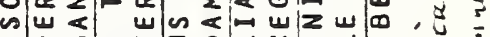

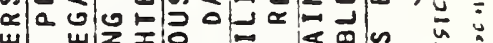

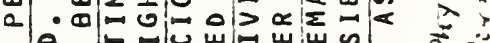
- $\therefore \propto ⿻ 上 丨$ (1)

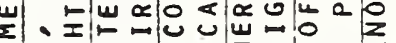

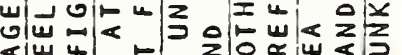

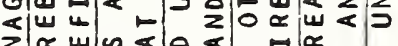
资| I $4=\omega$

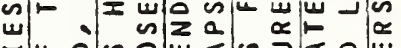

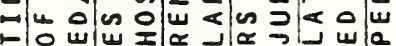
$\Rightarrow$ ग

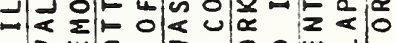

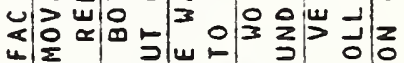

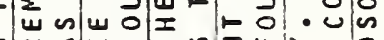
$v \propto<\omega\left|\sum u\right| 0$

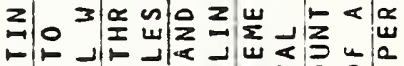

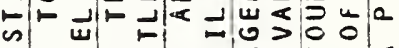

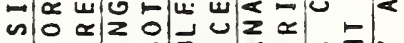
n)

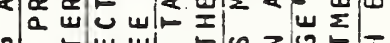
）.

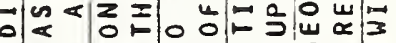

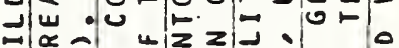
引군

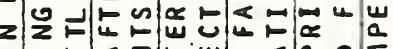

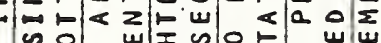

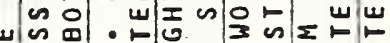

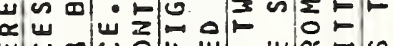

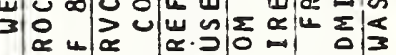
$\propto$ a

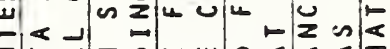

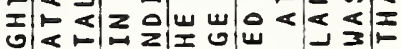
ज||

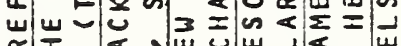

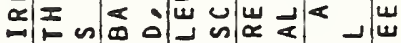

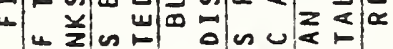
خ : i

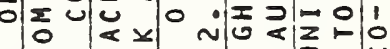

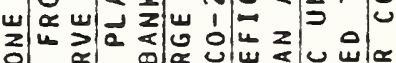

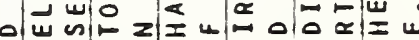
짔

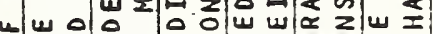
w

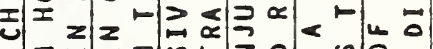

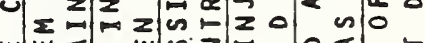

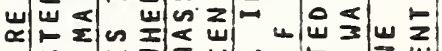

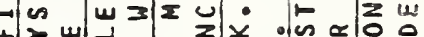
4 它 피

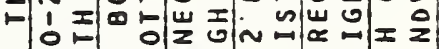
n N< ${ }^{\circ}=0$ = 0 is

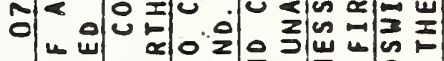

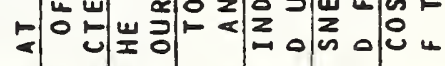




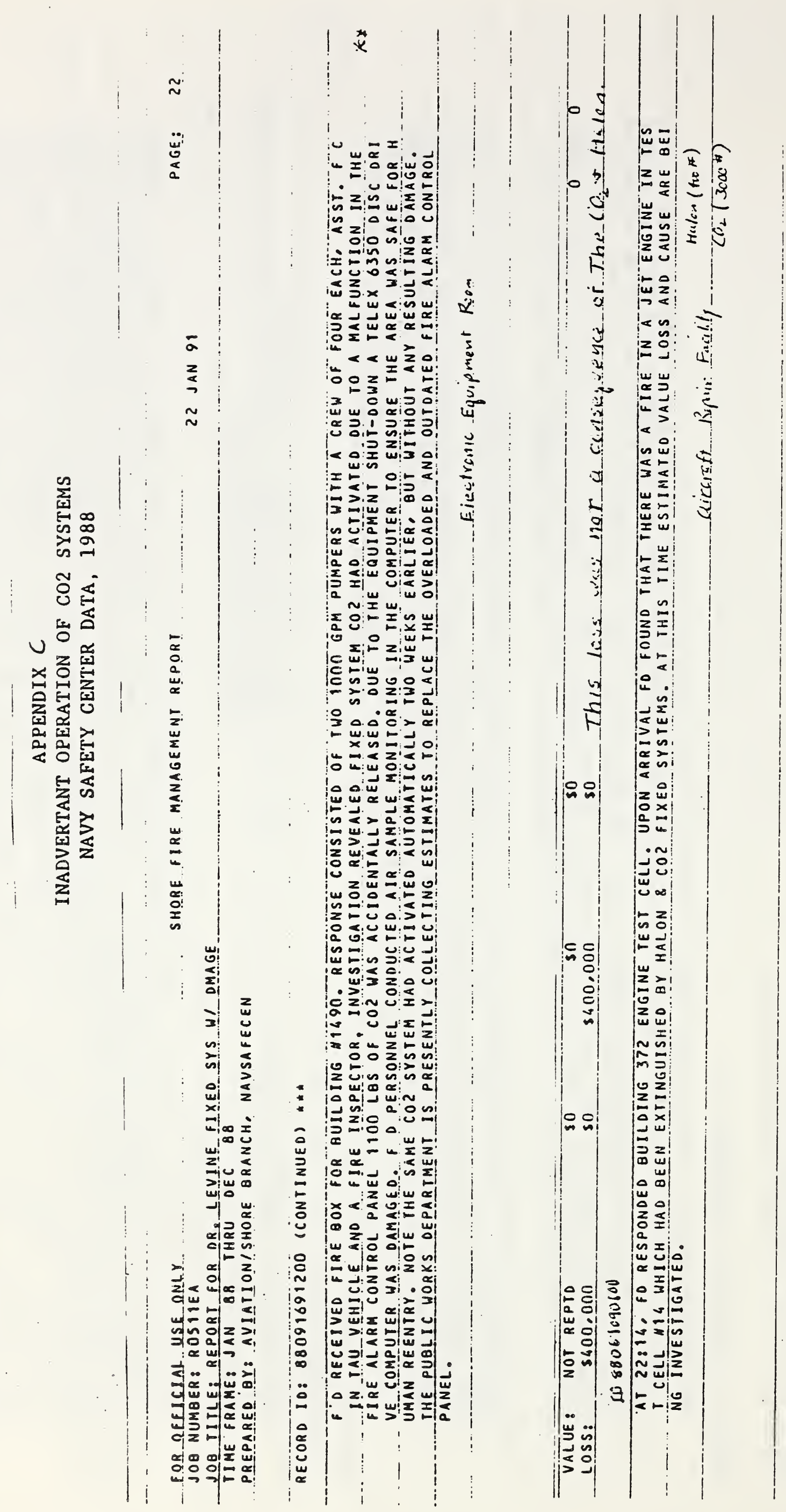




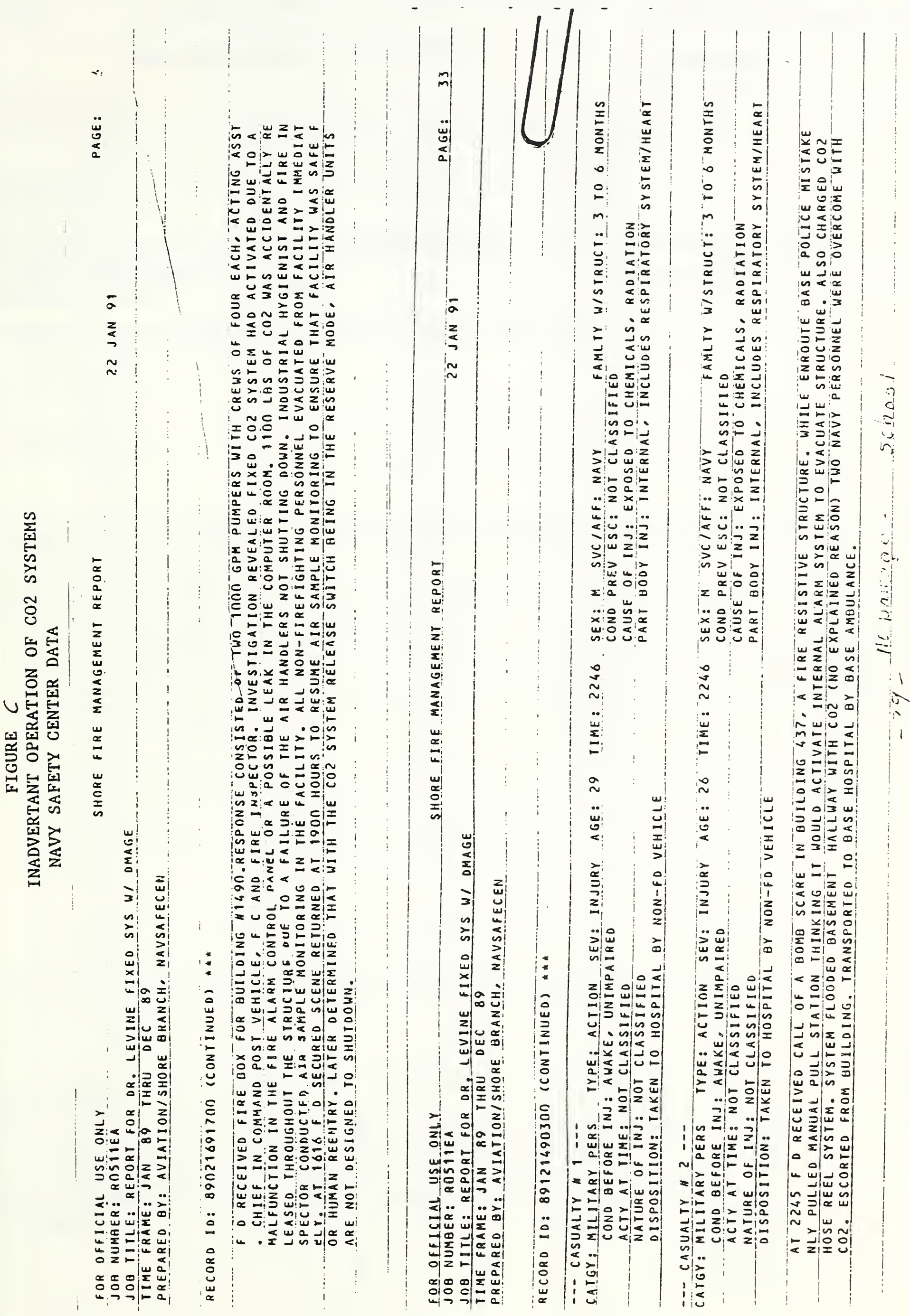




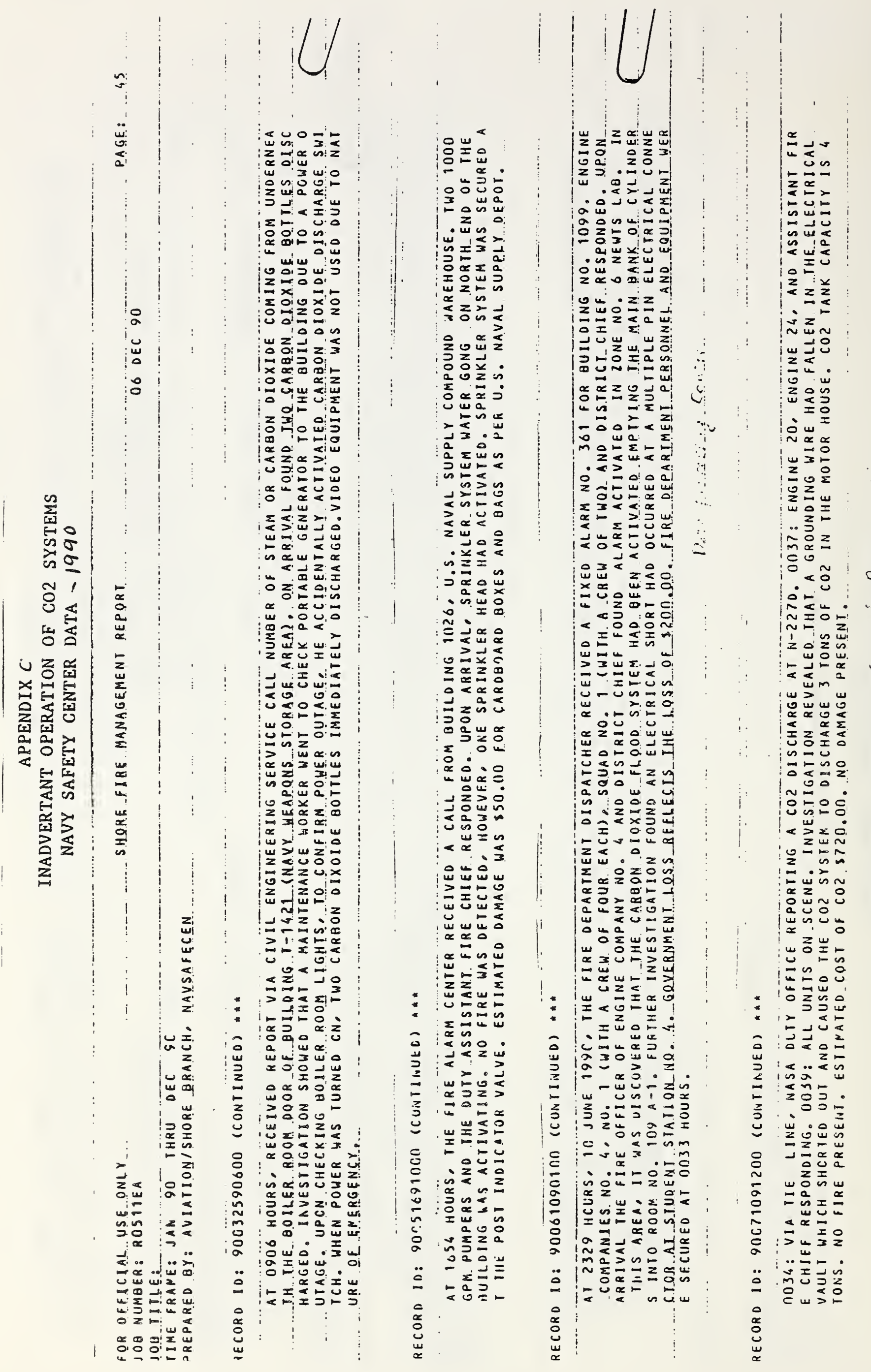




\begin{tabular}{|c|c|c|}
\hline \multirow[t]{3}{*}{$\begin{array}{l}\text { NIST-114A } \\
\text { (REY. 3-90) }\end{array}$} & \multirow{3}{*}{$\begin{array}{l}\text { U.S. DEPARTMENT OF COMMERCE } \\
\text { NATIONAL INSTITUTE OF STANDARDS AND TECHNOLOGY } \\
\text { BIBLIOGRAPHIC DATA SHEET }\end{array}$} & $\begin{array}{l}\text { 1. PUBUCATION OR REPORT NUMBER } \\
\text { NISTIR } 4620\end{array}$ \\
\hline & & 2. PERFORMINO ORGANIZATION REPORT HUMEEA \\
\hline & & $\begin{array}{l}\text { 3. PUBUCATION DATE } \\
\text { April } 1991\end{array}$ \\
\hline
\end{tabular}

4. TITLE AND SUBTITLE

Navy Safety Center Data on the Effects of Fire Protection Systems on Electrical Equipment

5. AUTHOR(S)

Robert S. Levine

6. PERFORMINO ORQANIZATION (IF JOINT OR OTHER THAN MIST, SEE INSTRUCTIONS)

U.S. DEPARTMEAT OF COMMERCE

MATIOHAL INSTITUTE OF STANDARDS AND TECHNOLOOY

CATHERSBURO, MO 2089

7. CONTRACT/ORANT MUMBER

8. TYPE OF REPORT AND PERIOD COVERED

9. SPONSORINO ORQANIZATION MAME AND COMPLTE ADDRESS (STREET, CITY, STATE, ZP)

Nuclear Regulatory Commission

Washington, DC

10. SUPPLEMENTARY NOTES

11. ABSTRACT (A 2OO-WORD OR LESS FACTUAL SUMMARY OF MOST SIGNIFICANT INFORMATION. FF DOCUMENT IHCLUDES A SIGNIFICANT BIBLUOGRAPMY OR UTERATURE SURVEY, MENTION TT HERE)

Records of the Navy Safety Center, Norfolk, VA were reviewed to find data relevant to inadvertant operation of installed Fire Extinguishing Systems in civilian Nuclear power plants. Navy data show the incidence of collateral fire or other damage by fresh water on operating electrical equipment in submarines, and in shore facilities is about the same as the civilian experience, about 30\%. Aboard surface ships, however, the collateral damage incidence is much lower, about $15 \%$. With sea water, the collateral damage incidence is at least $75 \%$. It is concluded that the fire extinguisher water has to be contaminated, as by rust in sprinkler systems or deposited salt spray, for most collateral damage to occur

Reasons for inadvertant operation (or advertant operation) of firex systems at shore facilities, submarines, and surface ships resemble those for nuclear power plants. Mechanical or electrical fallures lead the list, followed by mishaps during maintenance. Detector and alarm system failures are significant problems at navy shore facilities, and significant at nuclear power plants. Ships and submarines have few automatic firex systems, so this kind of failure is not significant. The Navy depends on trained firefighters to intervene. Prompt action with a portable 022 extinguisher is usually effective.

Fixed halon and $\mathrm{CO} 2$ systems in shore facilities cause no collateral damage.

Lists of individual Navy incidents with water and with halon and carbon dioxide are included as appendices to this report.

12. KEY WORDS (6 TO 12 ENTRIES; ALPHAEETICAL ORDER; CAPITALIZE OHLY PROPER MAMES; AND SEPARATE KEY WORDS BY SEMICOLOHS) carbon dioxide; collateral damage; electrical equipment; firex; halon; water

FOR OFFICLAL DISTRIBUTION. DO NOT RELEASE TO MATIONAL TECHMICAL INFORMATION SERVCE (NTIS).

ORDER FROM SUPERINTENDENT OF DOCUMENTS, U.S. GOVERNMENT PRINTINC OFFICE, WASHINOTON, DC 20402

ORDEA FROM MATIONAL TECMNICAL INFORMATION SEAVCE (NTIS), SPRIMCFIELD, VA 22161.

14. HUMBEA OF PRINTED PACES

84

15. PRICE A05 


